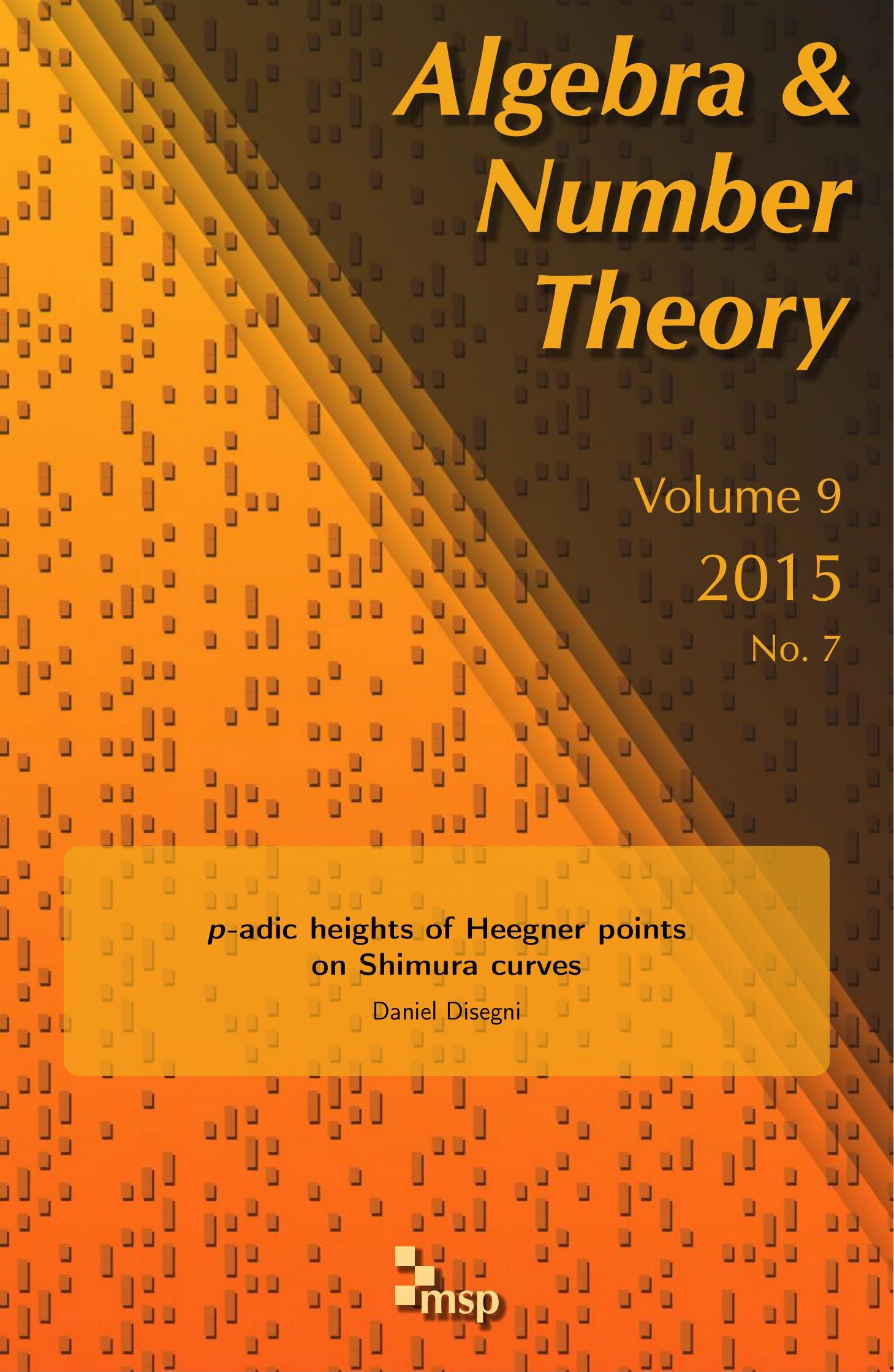




\title{
$p$-adic heights of Heegner points on Shimura curves
}

\author{
Daniel Disegni
}

Let $f$ be a primitive Hilbert modular form of parallel weight 2 and level $N$ for the totally real field $F$, and let $p$ be a rational prime coprime to $2 N$. If $f$ is ordinary at $p$ and $E$ is a $C M$ extension of $F$ of relative discriminant $\Delta$ prime to $N p$, we give an explicit construction of the $p$-adic Rankin-Selberg $L$-function $L_{p}\left(f_{E}, \cdot\right)$. When the sign of its functional equation is -1 , we show, under the assumption that all primes $\wp \mid p$ are principal ideals of $\mathcal{O}_{F}$ that split in $\mathscr{O}_{E}$, that its central derivative is given by the $p$-adic height of a Heegner point on the abelian variety $A$ associated with $f$.

This $p$-adic Gross-Zagier formula generalises the result obtained by PerrinRiou when $F=\mathbb{Q}$ and $(N, E)$ satisfies the so-called Heegner condition. We deduce applications to both the $p$-adic and the classical Birch and SwinnertonDyer conjectures for $A$.

A list of symbols can be found on page 1641.

Introduction

Part I. $\quad p$-adic $\boldsymbol{L}$-function and measures 1581

1. $\quad p$-adic modular forms

2. Theta measure 1590

3. Eisenstein measure 1599

4. The $p$-adic $L$-function 1604

Part II. Heights

5. $p$-adic heights and Arakelov theory

6. Heegner points on Shimura curves

7. Heights of Heegner points

Part III. Main theorem and consequences 1635

8. Proof of the main theorem 1635

9. Periods and the Birch and Swinnerton-Dyer conjecture 1639

List of symbols 1641

Acknowledgements 1643

References

MSC2010: primary 11G40; secondary 11F41, 11G18, 11F33, 11G50.

Keywords: Gross-Zagier, Heegner points, $p$-adic $L$-functions, Hilbert modular forms, $p$-adic heights,

Birch and Swinnerton-Dyer conjecture. 


\section{Introduction}

In this work, we generalise the $p$-adic analogue of the Gross-Zagier formula of [Perrin-Riou 1987] to totally real fields, in a generality similar to [Zhang 2001a; 2001b; 2004]. We describe here the main result and its applications.

The p-adic Rankin-Selberg L-function. Let $f$ be a primitive (that is, a normalised new eigenform) Hilbert modular form of parallel weight 2, level $N$ and trivial character for the totally real field $F$ of degree $g$ and discriminant $D_{F}$. Let $p$ be a rational prime coprime to $2 N$. Fix embeddings $\iota_{\infty}$ and $\iota_{p}$ of the algebraic closure $\overline{\mathbb{Q}}$ of $F$ into $\mathbb{C}$ and $\overline{\mathbb{Q}}_{p}$, respectively; we let $v$ denote the valuation on $\overline{\mathbb{Q}}_{p}$, normalised by $v(p)=1$.

Let $E \subset \overline{\mathbb{Q}}$ be a CM (that is, quadratic and purely imaginary) extension of $F$ of relative discriminant $\Delta$ coprime to $D_{F} N p$; let

$$
\varepsilon=\varepsilon_{E / F}: F_{\boldsymbol{A}}^{\times} / F^{\times} \rightarrow\{ \pm 1\}
$$

be the associated Hecke character and $\mathfrak{N}=N_{E / F}$ be the relative norm. If

$$
W: E_{A}^{\times} / E^{\times} \rightarrow \overline{\mathbb{Q}}^{\times}
$$

is a finite-order Hecke character ${ }^{1}$ of conductor $\mathfrak{f}=\mathfrak{f}(\mathcal{W})$ prime to $N \Delta$, the RankinSelberg $L$-function $L\left(f_{E}, \mathcal{W}, s\right)$ is the entire function defined for $\operatorname{Re} s>\frac{3}{2}$ by

$$
L\left(f_{E}, \mathcal{W}, s\right)=L^{N \Delta(\mathcal{W})}\left(\left.\varepsilon \mathcal{W}\right|_{F_{A}^{\times}}, 2 s-1\right) \sum_{m} \frac{a(f, m) r_{W}(m)}{N m^{s}},
$$

where $\Delta(\mathcal{W})=\Delta \mathfrak{N}(\mathfrak{f}), r_{\mathfrak{W}}(m)=\sum_{\mathfrak{N}(\mathfrak{a})=m} \mathscr{W}(\mathfrak{a})$ (the sum running over all nonzero ideals of $\mathrm{O}_{E}$ ) and

$$
L^{N \Delta(\mathcal{W})}\left(\left.\varepsilon^{\mathscr{W}}\right|_{\widehat{O}_{F}}, s\right)=\sum_{(m, N \Delta(\mathcal{W}))=1} \varepsilon(m) \mathcal{W}(m) N m^{-s} .
$$

This $L$-function admits a $p$-adic analogue (Section 4$)$. Let $E_{\infty}^{\prime}$ be the maximal abelian extension of $E$ unramified outside $p$ and $G^{\prime}=\operatorname{Gal}\left(E_{\infty}^{\prime} / E\right){ }^{2}$ (It has rank $1+\delta+g$ over $\mathbb{Z}_{p}$, where $\delta$ is the Leopoldt defect of $F$.) For each prime $\wp$ of $0_{F}$ dividing $p$, let

$$
P_{\wp, f}(X)=X^{2}-a(f, \wp) X+N \wp
$$

be the $\wp$-th Hecke polynomial of $f$, and assume that $v\left(\iota_{p}(a(f, \wp))\right)=0$; in this case, $f$ is said to be ordinary, and there is a unique root $\alpha_{\wp} \in \overline{\mathbb{Q}}$ of $P_{\wp}, f(X)$ such

\footnotetext{
${ }^{1}$ We will use the same notation throughout for a Hecke character, the associated ideal character and the associated Galois character.

${ }^{2}$ The reason for the notation is that later in the paper we will denote by $E_{\infty}$ the maximal $\mathbb{Z}_{p^{-}}$ subextension of $E_{\infty}^{\prime}$.
} 
that $\iota_{p}\left(\alpha_{\wp}\right)$ is a $p$-adic unit. Let $L \subset \overline{\mathbb{Q}}_{p}$ be the finite extension of $\mathbb{Q}_{p}$ generated by the Fourier coefficients $a(f, m)$ of $f$ and by the $\alpha_{\wp}$ for $\wp \mid p$.

Theorem A. There exists a unique element $L_{p}\left(f_{E}\right)$ of $\mathcal{O}_{L} \llbracket G^{\prime} \rrbracket \otimes_{\mathscr{O}_{L}} L$ satisfying the interpolation property

$$
L_{p}\left(f_{E}\right)(\mathcal{W})=\frac{\mathscr{W}\left(d_{F}^{(p)}\right) \tau(\bar{W}) N(\Delta(\mathcal{W}))^{1 / 2} V_{p}(f, \mathscr{W})^{\bar{W}}(\Delta)}{\alpha_{f} \Omega_{f}} L\left(f_{E}, \bar{W}, 1\right)
$$

for all finite-order characters ${ }^{W}$ of $\mathcal{G}^{\prime}$ of conductor $\mathfrak{f}(\mathcal{W})$. Here both sides are algebraic numbers, ${ }^{3} \overline{\mathcal{W}}=\mathcal{W}^{-1}$ and

$$
\Omega_{f}=\left(8 \pi^{2}\right)^{g}\langle f, f\rangle_{N}
$$

with $\langle\cdot, \cdot\rangle_{N}$ the Petersson inner product $(1.1 .2) ; \tau(\bar{W})$ is a normalised Gauss sum; $V_{p}(f, \mathcal{W})$ is a product of partial Euler factors at $p$; and finally $\alpha_{\mathfrak{f}}=\prod_{\wp \mid p} \alpha_{\wp}^{v_{\wp}(\mathfrak{N}(\mathfrak{f}))}$.

This is essentially a special case of [Panchishkin 1988; Hida 1991]; we reprove it entirely here (see Section 4, especially Theorem 4.3.4) because the precise construction of $L_{p}\left(f_{E}\right)$ will be crucial for us. It is obtained, using a technique of Hida and Perrin-Riou, by applying a $p$-adic analogue of the functional "Petersson product with $f$ " to a convolution $\Phi$ of Eisenstein and theta measures on $\varphi^{\prime}$ valued in $p$-adic modular forms (so that $\Phi=\Phi(\mathscr{W})$ is an analogue of the kernel of the classical Rankin-Selberg convolution). The approach we follow is adelic; one novelty introduced here is that the theta measure is constructed via the Weil representation, which seems very natural and would generalise well to higher-rank cases.

On the other hand, Manin [1976], Dimitrov [2013] and others have constructed a $p$-adic $L$-function $L_{p}(f, \cdot) \in \mathcal{O}_{L} \llbracket \varphi_{F} \rrbracket$ as an analogue of the standard $L$-function $L(f, s)$, where $\mathscr{G}_{F}^{\prime}$ is the Galois group of the maximal abelian extension of $F$ unramified outside $p$; it is characterised by the interpolation property

$$
L_{p}(f, \chi)=\chi\left(d_{F}^{(p)}\right) \frac{\tau(\bar{\chi}) N(\mathfrak{f}(\chi))^{1 / 2}}{\alpha_{\mathfrak{f}(\chi)}} \frac{L(f, \bar{\chi}, 1)}{\Omega_{f}^{+}}
$$

for all finite-order characters $\chi$ of conductor $\mathfrak{f}(\chi)$ that are trivial at infinity and ramified at all primes $v \mid p$. (Here $\Omega_{f}^{+}$is a suitable period (see Section 9.1) and $\tau(\chi)$ is again a normalised Gauss sum.) The corresponding formula for complex $L$-functions implies a factorisation (4.4.1)

$$
L_{p}\left(f_{E}, \chi \circ \mathfrak{N}\right)=\chi(\Delta)^{2} \frac{\Omega_{f}^{+} \Omega_{f_{\varepsilon}}^{+}}{D_{E}^{-1 / 2} \Omega_{f}} L_{p}(f, \chi) L_{p}\left(f_{\varepsilon}, \chi\right),
$$

where $f_{\varepsilon}$ is the form with coefficients $a\left(f_{\varepsilon}, m\right)=\varepsilon(m) a(f, m)$ and $D_{E}=N(\Delta)$.

\footnotetext{
${ }^{3}$ By a well-known theorem of [Shimura 1978]. They are compared via $\iota_{p}^{-1}$ and $\iota_{\infty}^{-1}$.
} 
Heegner points on Shimura curves and the main theorem. Suppose that $\varepsilon(N)=$ $(-1)^{g-1}$, where $g=[F: \mathbb{Q}]$. Then for each embedding $\tau: F \rightarrow \mathbb{C}$, there is a quaternion algebra $B(\tau)$ over $F$ ramified exactly at the finite places $v \mid N$ for which $\varepsilon\left(N_{v}\right)=-1$ and the infinite places different from $\tau$; it admits an embedding $\rho: E \hookrightarrow B(\tau)$, and we can consider an order $R$ of $B(\tau)$ of discriminant $N$ and containing $\rho\left(\mathbb{O}_{E}\right)$. These data define a Shimura curve $X$. It is an algebraic curve over $F$, whose complex points for any embedding $\tau: F \rightarrow \mathbb{C}$ are described by

$$
X\left(\mathbb{C}_{\tau}\right)=B(\tau)^{\times} \backslash \mathfrak{H}^{ \pm} \times \widehat{B}(\tau)^{\times} / \widehat{F}^{\times} \widehat{R}^{\times} \cup\{\text { cusps }\} .
$$

It plays the role of the modular curve $X_{0}(N)$ in the works of Gross and Zagier [1986] and Perrin-Riou [1987], who consider the case $F=\mathbb{Q}$ and $\varepsilon(v)=1$ for all $v \mid N$ (it is only in this case that the set of cusps is not empty).

The curve $X$ is connected but not geometrically connected. Let $J(X)$ be its Albanese (@ Jacobian) variety; it is an abelian variety defined over $F$, geometrically isomorphic to the product of the Albanese varieties of the geometrically connected components of $X$. There is a natural map $\iota: X \rightarrow J(X) \otimes \mathbb{Q}$ given by $\iota(x)=[x]-[\xi]$, where $[\xi] \in \mathrm{Cl}(X) \otimes \mathbb{Q}$ is a canonical divisor class constructed in [Zhang 2001a] having degree 1 in every geometrically connected component of $X$; an integer multiple of $\iota$ gives a morphism $X \rightarrow J(X)$ defined over $F$.

As in the modular curve case, the curve $X$ admits a finite collection of Heegner points defined over the Hilbert class field $H$ of $E$ and permuted simply transitively by $\operatorname{Gal}(H / E)$. They are the points represented by $\left(x_{0}, t\right)$ for $t \in \widehat{E}^{\times} / E^{\times} \widehat{F}^{\times} \hat{O}_{E}^{\times}$ when we use the complex description above and view $E \subset B$ via $\rho$. We let $y$ be any such Heegner point, and let $[z]$ denote the class

$$
[z]=u^{-1} \iota\left(\operatorname{Tr}_{H / E} y\right) \in J(X)(E) \otimes \mathbb{Q},
$$

where $u=\left[\mathbb{O}_{E}^{\times}: \mathbb{O}_{F}^{\times}\right]$.

As a consequence of Jacquet-Langlands theory, the Hecke algebra on Hilbert modular forms of level $N$ acts through its quaternionic quotient on $J(X)$. Let $z_{f} \in J(X)(E) \otimes \overline{\mathbb{Q}}$ be the $f$-component of $[z]$.

Heights and the formula. On any curve $X$ over a number field $E$, there is a notion (Section 5.2) of $p$-adic height $\langle\cdot, \cdot\rangle_{\ell}$ attached to the auxiliary choices of splittings of the Hodge filtrations on $H_{\mathrm{dR}}^{1}\left(X / E_{w}\right)$ for $w \mid p$ and of a $p$-adic logarithm $\ell: E_{A}^{\times} / E^{\times} \rightarrow \mathbb{Q}_{p}$. It is a symmetric bilinear pairing on the group of degree-0 divisors on $X$ modulo rational equivalence, which we can view as a pairing on $J(X)(E)$. More generally, for any abelian variety $A / E$, there is defined a $p$-adic height pairing on $A(E) \times A^{\vee}(E)$. In our case, there is a canonical choice for the Hodge splittings on the $f$-components of the Albanese variety $J(X)$, given by the unit root subspaces, and we choose our height pairing on $J(X)$ to be compatible with this choice. 
Under the assumption $\varepsilon(N)=(-1)^{g-1}$, the value $L_{p}\left(f_{E}, \mathbb{1}\right)$ is zero by the complex functional equation and the interpolation property; in fact, we have more generally $L_{p}\left(f_{E}, \mathcal{W}\right)=0$ for any anticyclotomic character $\mathcal{W}$ of $\mathscr{G}$. We can then consider its derivative in a cyclotomic direction. Let thus $\mathcal{W}$ be a Hecke character of $E$ induced from a Hecke character of $F$ taking values in $1+p \mathbb{Z}_{p} \subset \mathbb{Z}_{p}^{\times}$, and assume $\mathcal{W}$ is ramified at all places dividing $p$. The derivative of $L_{p}\left(f_{E}\right)$ in the $W$-direction is

$$
L_{p, W}^{\prime}\left(f_{E}, \mathbb{1}\right)=\left.\frac{d}{d s}\right|_{s=0} L_{p}\left(f_{E}\right)\left(W^{s}\right) .
$$

Theorem B. Assume that $\Delta_{E / F}$ is totally odd and that every prime $\wp \mid p$ is a principal ideal in $\mathrm{O}_{F}$ and splits in $\mathrm{O}_{E}$. Suppose that $\varepsilon_{E / F}(N)=(-1)^{g-1}$. Then $L_{p}\left(f_{E}, \mathbb{1}\right)=0$ and

$$
L_{p, W}^{\prime}\left(f_{E}, \mathbb{1}\right)=D_{F}^{-2} \prod_{\wp}\left(1-\frac{1}{\alpha_{\wp}}\right)^{2}\left(1-\frac{1}{\varepsilon(\wp) \alpha_{\wp}}\right)^{2}\left\langle z_{f}, z_{f}\right\rangle_{W}
$$

where $\langle\cdot, \cdot\rangle_{\text {W }}$ is the height pairing on $J(X)(E)$ associated with the logarithm $\ell=\left.\frac{d}{d s}\right|_{s=0} W^{s}$.

The hypothesis that the primes $\wp \mid p$ are principal is a technical assumption that intervenes only in Proposition 8.1.1. ${ }^{4}$ The assumption that they split in $E$ is essential to the argument, but like the assumption on $\Delta_{E / F}$, it can be removed a posteriori if the left-hand side of the formula below is nonzero-see Section 8.2.

Applications to the conjecture of Birch and Swinnerton-Dyer. It is conjectured that to any Hilbert modular newform $f$ one can attach a simple abelian variety $A=A_{f}$ over $F$, characterised uniquely up to isogeny ${ }^{5}$ by the equality of $L$-functions

$$
L(A, s)=\prod_{\sigma: M_{f} \rightarrow \mathbb{C}} L\left(f^{\sigma}, s\right) .
$$

Here $M=M_{f}$ is the field generated by the Fourier coefficients of $f ; A$ has dimension $[M: \mathbb{Q}]$, and its endomorphism algebra contains $M$ (we say that $A$ is of $\mathrm{GL}_{2}(M)$-type; in fact since $F$ is totally real, $A$ is of strict $\mathrm{GL}_{2}$-type; that is, its endomorphism algebra equals $M$ - see, e.g., [Yuan et al. 2013, Lemma 3.3]). The conjecture is known to be true [Zhang 2001a, Theorem B] when

$$
\text { either }[F: \mathbb{Q}] \text { is odd or } v(N) \text { is odd for some finite place } v
$$

(the assumptions of Theorem B above imply that one of these conditions holds); in this case, $A$ is a quotient $\phi$ of $J(X)$ for a suitable Shimura curve $X$ of the type

\footnotetext{
${ }^{4} \mathrm{~A}$ somewhat more sophisticated approach to our main result should remove this and other restrictions [Disegni 2015].

${ }^{5}$ Thanks to Faltings's isogeny theorem [1983].
} 
described above. Vice versa, any abelian variety of $\mathrm{GL}_{2}$-type (for some field $M$ ) over a totally real field $F$ is conjectured to be associated with a Hilbert modular form $f$ as above. This is known to be true for all elliptic curves $A$ over $F$ when $F$ is $\mathbb{Q}$ or a real quadratic field and for all but possibly finitely many geometric isomorphism classes if $F$ is a general totally real field (see [Le Hung 2014], whose result is somewhat stronger than this, and [Freitas et al. 2015]; the results build on the method of Wiles for $F=\mathbb{Q}$ ).

In view of known $\operatorname{Aut}(\mathbb{C} / \mathbb{Q})$-equivariance properties of automorphic $L$-functions and the above equality, the order of vanishing of $L(A, s)$ at $s=1$ will be an integer multiple $r[M: \mathbb{Q}]$ of the dimension of $A$. We call $r$ the $M$-order of vanishing of $L(A, s)$ or the analytic $M$-rank of $A$.

Conjecture (Birch and Swinnerton-Dyer). Let $A$ be an abelian variety of $\mathrm{GL}_{2}(M)$ type over a totally real field $F$ of degree $g$.

(1) The $M$-order of vanishing of $L(A, s)$ at $s=1$ is equal to the dimension of $A(F)_{\mathbb{Q}}$ as $M$-vector space.

(2) The Tate-Shafarevich group $\amalg(A / F)$ is finite, and the leading term of $L(A, s)$ at $s=1$ is given by

$$
\frac{L^{*}(A, 1)}{\Omega_{A}}=D_{F}^{-d / 2}|\amalg(A / F)| R_{A} \prod_{v \nmid \infty} c_{v}=\operatorname{BSD}(A),
$$

where $d=\operatorname{dim} A=[M: \mathbb{Q}]$, the $c_{v}$ are the Tamagawa numbers of $A$ at finite places (almost all equal to 1 ),

$$
\Omega_{A}=\prod_{\tau: F \rightarrow \mathbb{R}} \int_{A\left(\mathbb{R}_{\tau}\right)}\left|\omega_{A}\right|_{\tau}
$$

for a Néron differential ${ }^{6} \omega_{A}$ and

$$
R_{A}=\frac{\operatorname{det}\left\langle x_{i}, y_{j}\right\rangle}{\left[A(F): \sum \mathbb{Z} x_{i}\right]\left[A^{\vee}(F): \sum \mathbb{Z} y_{j}\right]}
$$

is the regulator of the Néron-Tate height paring on $A(F) \times A^{\vee}(F)$, defined using any subsets $\left\{x_{i}\right\}$ and $\left\{y_{j}\right\}$ of $A(F)$ and $A^{\vee}(F)$ inducing bases of $A(F)_{\mathbb{Q}}$ and $A^{\vee}(F)_{\mathbb{Q}}$.

By the automorphic description of $L(A, s)$ and [Shimura 1978], we know that $L(A, s) / \prod_{\sigma: M_{f} \rightarrow \mathbb{C}} \Omega_{f^{\sigma}}^{+}$is an algebraic number. Comparison with the Birch and Swinnerton-Dyer conjecture suggests the following conjecture:

${ }^{6}$ When it exists, which is only guaranteed if $F=\mathbb{Q}$. Otherwise, we take for $\omega_{A}$ any generator of $H^{0}\left(A, \Omega_{A / F}^{d}\right)$ and to define $\Omega_{A}$ we divide by the product of the indices $\left[H^{0}\left(\mathscr{A}_{v}, \Omega_{\mathbb{A}_{v} / \mathcal{O}_{F, v}}\right)\right.$ : $\left.\mathcal{O}_{F, v} \widetilde{\omega_{A}}\right]$ of (the extension of) $\omega_{A}$ in the space of top differentials on the local Néron models $\mathscr{A}_{v} / \mathcal{O}_{F, v}$ of $A$. 
Conjecture (period conjecture). We have

$$
\Omega_{A} \sim \prod_{\sigma: M_{f} \rightarrow \mathbb{C}} \Omega_{f^{\sigma}}^{+} \text {in } \mathbb{C}^{\times} / \overline{\mathbb{Q}}^{\times} .
$$

The conjecture is known for $F=\mathbb{Q}$ [Shimura 1981] or when $A$ has complex multiplication (over $\overline{\mathbb{Q}}$ ) [Blasius 1986]; see Section 9 below for a more precise conjecture and some further evidence and motivation.

Assuming the conjecture, we can define a $p$-adic $L$-function $L_{p}(A)$ for $A$ by

$$
L_{p}(A)=\frac{\prod_{\sigma} \Omega_{f^{\sigma}}^{+}}{\Omega_{A}} \prod_{\sigma: M_{f} \rightarrow \mathbb{C}} L_{p}\left(f^{\sigma}\right)
$$

for any prime $p$ such that $A$ has good ordinary reduction at all primes above $p$.

Then, fixing a ramified Hecke character $v: \mathscr{G}_{F}^{\prime} \rightarrow 1+p \mathbb{Z}_{p} \subset \mathbb{Z}_{p}^{\times}$that we omit from the notation, one can formulate a $p$-adic version of the Birch and Swinnerton-Dyer conjecture similarly as above for $L_{p}\left(A, v^{s}\right):{ }^{7}$ the conjectural formula reads

$$
\prod_{\wp \mid p}\left(1-\alpha_{\wp}^{-1}\right)^{-2} L_{p}^{*}(A, \mathbb{1})=\operatorname{BSD}_{p}(A)
$$

where $\mathrm{BSD}_{p}(A)$ differs from $\mathrm{BSD}(A)$ only in the regulator term, which is now the regulator of the $p$-adic height pairing on $A(F) \times A^{\vee}(F)$ associated with the $p$-adic logarithm $\ell$ deduced from $v$ as in Theorem B. One can also formulate a main conjecture of Iwasawa theory for $L_{p}(A)$ [Schneider 1985].

Then, just as in [Perrin-Riou 1987], we can deduce the following arithmetic application of Theorem B:

Theorem C. Assume the period conjecture holds for the abelian variety $A=A_{f}$ and that $A$ satisfies $(*)$. For an ordinary prime $p>2$ decomposing into principal prime ideals in $\mathrm{O}_{F}$ :

(1) The following are equivalent:

(a) The p-adic L-function $L_{p}\left(A, v^{s}\right)$ has $M_{f}$-order of vanishing $r \leq 1$ at the central point.

(b) The complex L-function $L(A, s)$ has $M_{f}$-order of vanishing $r \leq 1$ at the central point, and the p-adic height pairing associated with $v$ is nonvanishing on $A(F)$.

(2) If either of the above assumptions holds, the rank parts of the classical and the p-adic Birch and Swinnerton-Dyer conjecture are true for $A$ and the Tate-Shafarevich group of A is finite.

\footnotetext{
${ }^{7}$ Here $s \in \mathbb{Z}_{p}$ and the central point is $s=0$, corresponding to $v^{0}=\mathbb{1}$.
} 
(3) If moreover the cyclotomic Iwasawa main conjecture is true for A, then the classical and the p-adic Birch and Swinnerton-Dyer formulas for A are true up to a p-adic unit.

Proof. In part (1), the statement follows trivially from the construction of $L_{p}(A)$ if $r=0$; if $r=1$, both conditions are equivalent to the assertion that, for a suitable $\mathrm{CM}$ extension $E$, the Heegner point $z_{f}=z_{f, E}$ is nontorsion: this is obvious from our main theorem in case (a); in case (b), by [Zhang 2001a; 2001b] (generalising [Gross and Zagier 1986; Kolyvagin 1988; Kolyvagin and Logachëv 1991]), the Heegner point

$$
P=\sum_{\sigma} \operatorname{Tr}_{E / F} \phi\left(z_{f^{\sigma}, E}\right) \in A(F) \otimes \mathbb{Q}
$$

(with $\phi: J(X) \rightarrow A$ ) generates $A(F) \otimes \mathbb{Q}$ as $M_{f}$-vector space so that the $p$-adic height pairing on $A(F)$ is nonvanishing if and only if it is nonzero at $z_{f}$. Part (2) then follows from (1) and [Zhang 2001a; 2001b].

Schneider [1985] proves an "arithmetic" version of the $p$-adic Birch-SwinnertonDyer formula for (the Iwasawa $L$-function associated with) $A$, which under the assumption of (3) can be compared to the analytic $p$-adic formula as explained in [Perrin-Riou 1987] to deduce the $p$-adic Birch and Swinnerton-Dyer formula up to a $p$-adic unit. In the analytic rank- 0 case, the classical Birch and Swinnerton-Dyer formula follows immediately. In the case of analytic rank 1, recall that the main result of [Zhang 2001a; 2004] is, in our normalisation, the formula

$$
\frac{L^{\prime}\left(f_{E}, 1\right)}{\Omega_{f}}=\frac{1}{D_{F}^{2} D_{E}^{1 / 2}}\left\langle z_{f}, z_{f}\right\rangle=: D_{E}^{-1 / 2} \mathrm{GZ}\left(f_{E}\right)
$$

(where $\langle\cdot, \cdot\rangle$ denotes the Néron-Tate height), whereas we introduce the notation $\mathrm{GZ}_{p}\left(f_{E}\right)$ to write our formula (for any fixed ramified cyclotomic character $\mathscr{W}=$ $\nu \circ \mathfrak{N})$ as

$$
L_{p}^{\prime}\left(f_{E}, \mathbb{1}\right)=\prod_{\wp \mid p}\left(1-\frac{1}{\alpha_{\wp}}\right)^{2}\left(1-\frac{1}{\varepsilon(\wp) \alpha_{\wp}}\right)^{2} \mathrm{GZ}_{p}\left(f_{E}\right) .
$$

Then, after choosing $E$ suitably so that $L\left(f_{\varepsilon}, 1\right) \neq 0$ (which can be done by [Bump et al. 1990; Waldspurger 1985]), we can argue as in [Perrin-Riou 1987] to compare the $p$-adic and the complex Birch and Swinnerton-Dyer formulas via the corresponding Gross-Zagier formulas to get the result. Namely, we have

$$
\begin{aligned}
\frac{L^{*}(A, 1)}{\Omega_{A} \operatorname{BSD}(A)} & =\frac{\prod_{\sigma} \Omega_{f^{\sigma}}^{+}}{\Omega_{A}} \frac{1}{\operatorname{BSD}(A)} \prod_{\sigma} \frac{L^{\prime}\left(f_{E}^{\sigma}, 1\right)}{\Omega_{f^{\sigma}}} \frac{\Omega_{f^{\sigma}}}{\Omega_{f^{\sigma}}^{+} \Omega_{f_{\varepsilon}^{\sigma}}^{+}} \frac{\Omega_{f_{\varepsilon}^{\sigma}}^{+}}{L\left(f_{\varepsilon}^{\sigma}, 1\right)} \\
& =\frac{\prod_{\sigma} \Omega_{f^{\sigma}}^{+}}{\Omega_{A}} \frac{\prod_{\sigma} \operatorname{GZ}\left(f_{E}^{\sigma}\right)}{\operatorname{BSD}(A)} \prod_{\sigma} \frac{D_{E}^{-1 / 2} \Omega_{f^{\sigma}}}{\Omega_{f^{\sigma}}^{+} \Omega_{f_{\varepsilon}^{\sigma}}^{+}} \frac{\Omega_{f_{\varepsilon}^{\sigma}}^{+}}{L\left(f_{\varepsilon}^{\sigma}, 1\right)}
\end{aligned}
$$


by the complex Gross-Zagier formula and the factorisation of $L\left(f_{E}, s\right)$. Similarly,

$$
\prod_{\wp \mid p}\left(1-\alpha_{\wp}^{-1}\right)^{-2} \frac{L_{p}^{*}(A, 1)}{\operatorname{BSD}_{p}(A)}=\frac{\prod_{\sigma} \Omega_{f^{\sigma}}^{+}}{\Omega_{A}} \frac{\prod_{\sigma} \mathrm{GZ}_{p}\left(f_{E}^{\sigma}\right)}{\operatorname{BSD}_{p}(A)} \prod_{\sigma} \frac{D_{E}^{-1 / 2} \Omega_{f^{\sigma}}}{\Omega_{f^{\sigma}}^{+} \Omega_{f_{\varepsilon}^{\sigma}}^{+}} \frac{\Omega_{f_{\varepsilon}^{\sigma}}^{+}}{L\left(f_{\varepsilon}^{\sigma}, 1\right)}
$$

by the $p$-adic Gross-Zagier formula, the factorisation of $L_{p}\left(f_{E}\right)$ and the interpolation property of $L_{p}\left(f_{\varepsilon}\right)$. Since we are assuming to know that the left-hand side of the last formula is a $p$-adic unit, the result follows from observing the equality

$$
\frac{\prod_{\sigma} \mathrm{GZ}\left(f_{E}^{\sigma}\right)}{\operatorname{BSD}(A)}=\frac{\prod_{\sigma} \mathrm{GZ}_{p}\left(f_{E}^{\sigma}\right)}{\mathrm{BSD}_{p}(A)}
$$

of rational numbers. ${ }^{8}$

Alternative approaches to the Birch and Swinnerton-Dyer formula in rank 1 have recently been proposed, at least for the case $F=\mathbb{Q}$, by Wei Zhang [2014] and Xin Wan.

Discussion of the assumptions. The conjecture on periods could be dispensed of if one were willing to work with a "wrong" $p$-adic $L$-function for $A$ (namely, one without the period ratio appearing in the definition above). Then at least the rank part of the $p$-adic Birch and Swinnerton-Dyer conjecture makes sense and parts (1) and (2) of Theorem $\mathrm{C}$ hold. The nonvanishing of the $p$-adic height pairing is only known for CM elliptic curves [Bertrand 1984]. The Iwasawa main conjecture is known in most cases for ordinary elliptic curves over $\mathbb{Q}$ thanks to the work of Rubin, Kato and Skinner and Urban [2014]. For Hilbert modular forms, one divisibility in the CM case is proved by [Hsieh 2014]; results on the general case are obtained by [Wan 2013]. We can then record the following unconditional result, whose assumptions are inherited from [Hsieh 2014]:

Theorem D. Let $A / F$ be an elliptic curve with complex multiplication by the ring of integers $0_{K}$ of an imaginary quadratic field $K$. Let $K^{\prime}=F K$, and let $h_{K^{\prime}}^{-}=h_{K^{\prime}} / h_{K}$ be the relative class number of $K^{\prime} / F$. Let $p \nmid 6 h_{K^{\prime}}^{-} D_{F}$ be a prime such that, for all primes $\wp \mid p, \wp$ is principal and $A$ has good ordinary reduction at $\wp$. Suppose that A satisfies $(*)$ and that $\operatorname{ord}_{s=1} L(A, s) \leq 1$. Then

$$
v_{p}\left(\frac{L^{*}(A, 1)}{R_{A} \Omega_{A}}\right) \leq v_{p}\left(|\amalg(A / F)| \prod_{v \nmid \infty} c_{v}\right) .
$$

\footnotetext{
${ }^{8}$ The rationality of the ratios follows from the fact that the $z_{f} \sigma$ essentially belong to $J(X)(F)$ that is, they belong to the +1-eigenspace for the action of $\operatorname{Gal}(E / F)$ on $J(X)(E) \otimes \overline{\mathbb{Q}}$ - and that, in this sense, their images $\phi\left(z_{f^{\sigma}}\right)$ form a $\operatorname{Gal}(\overline{\mathbb{Q}} / \mathbb{Q})$-invariant basis of $A(F) \otimes \overline{\mathbb{Q}}$, orthogonal for the height pairing.
} 
Results toward the divisibility in the opposite direction can be obtained from the method of Kolyvagin; see [Kolyvagin 1991] (for $F=\mathbb{Q}$ ) and [Howard 2004] (for general $F$ but excluding the CM case).

Plan of the proof. The proof of the main formula follows the strategy of [PerrinRiou 1987]. It is enough (see Section 8) to study the case where $W$ is cyclotomic $\left(W=W^{c}\right)$ since both sides of the formula are zero when $\mathscr{W}$ is anticyclotomic $\left(W^{c} W^{c}=\mathbb{1}\right)$.

In the first part of this paper, we construct the measure $\Phi$ on $\mathscr{G}$ valued in $p$-adic modular forms such that $L_{p}\left(f_{E}\right)(\mathcal{W})$ essentially equals $l_{f_{\alpha}}(\Phi(\mathcal{W}))$, where $l_{f_{\alpha}}$ is a $p$-adic analogue of the functional "Petersson product with $f$ " on $p$-adic modular forms. This allows us to write

$$
L_{p, W}^{\prime}\left(f_{E}, \mathbb{1}\right) \doteq l_{f_{\alpha}}\left(\Phi_{\mathscr{W}}^{\prime}\right),
$$

where $\doteq$ denotes equality up to suitable nonzero factors and $\Phi_{\mathscr{W}}^{\prime}=\left.\frac{d}{d s}\right|_{s=0} \Phi\left(\mathcal{W}^{s}\right)$ is a $p$-adic Hilbert modular form.

On the other hand, there is a modular form $\Psi$ with Fourier coefficients given by $\langle z, T(m) z\rangle_{W}$ so that $l_{f_{\alpha}}(\Psi) \doteq\left\langle z_{f}, z_{f}\right\rangle_{\mathscr{W}}$. It can be essentially written as a sum of modular forms $\Psi_{\text {fin }}+\Psi_{p}$, where $\Psi_{\text {fin }}$ encodes the local contributions to the height from places not dividing $p$ and $\Psi_{p}=\sum \Psi_{\wp}$, the contribution from the places $\wp$ above $p$. Then we can show by explicit computation that the Fourier coefficients of $\Phi^{\prime}$ are equal to the Fourier coefficients of $\Psi_{\text {fin }}$ up to the action of suitable Hecke operators at $p$. The desired formula then follows once we show that $l_{f_{\alpha}}\left(\Psi_{p}\right)$ vanishes. To prove this, we examine the effect of the operator $U_{\wp}$ on $\Psi_{\wp}$ and find that, in a suitable quotient space, the ordinary projection of $\Psi_{\wp}$ is zero. The study of $\Psi_{\wp}$ follows the methods of Perrin-Riou.

One difficulty in the approach just outlined is that compared to the case of modular curves there are no cusps available so that in this case the divisors $z$ and $T(m) z$ have intersecting supports and the decomposition of the height pairing into a sum of local pairings is not available. Our solution to this problem, which is inspired by [Zhang 2001a], is to make use of $p$-adic Arakelov theory as developed by [Besser 2005] (see Section 5.3) and work consistently in a suitable quotiented space of Fourier coefficients.

Perspective. The original Gross-Zagier formula has undergone an impressive transformation since its first appearance in 1986, culminating in the recent book of Yuan, Zhang and Zhang [Yuan et al. 2013]. Obviously, this work is only a first step in catching up on the $p$-adic side. ${ }^{9}$ The latter has also seen important developments, with generalisations to the nonordinary case, by [Kobayashi 2013] and, to the case

\footnotetext{
${ }^{9}$ For a more accomplished attempt, see [Disegni 2015].
} 
of higher weights, by [Nekováŕ 1995; Shnidman 2014]. It would certainly be of interest to generalise those results to the setting of the present work. ${ }^{10}$

Results similar to those presented here were recently obtained in the thesis of Li Ma (Paris 6).

\section{Part I. $p$-adic $L$-function and measures}

This part is dedicated to the construction of the measure giving the $p$-adic RankinSelberg $L$-function $L_{p}\left(f_{E}\right)$ and to the computation of its Fourier coefficients.

\section{1. $p$-adic modular forms}

1.1. Hilbert modular forms. Let us define compact subgroups of $G L_{2}\left(A^{\infty}\right)$ :

- $K_{0}(N)=\left\{\left(\begin{array}{ll}a & b \\ c & d\end{array}\right) \in \boldsymbol{G L}_{2}\left(\hat{\mathscr{O}}_{F}\right) \mid c \equiv 0 \bmod N \hat{\mathscr{O}}_{F}\right\}$ if $N$ is an ideal of $\hat{O}_{F}$,

- $K_{1}(N)=\left\{\left(\begin{array}{ll}a & b \\ c & d\end{array}\right) \in K_{0}(N) \mid a \equiv 1 \bmod N \hat{0}_{F}\right\}$.

Let $k$ be an element of $\mathbb{Z}_{\geq 0}^{\operatorname{Hom}(F, \overline{\mathbb{Q}})}$ and $\psi$ be a character of $F_{A}^{\times} / F^{\times}$of conductor dividing $N$ satisfying $\psi_{v}(-1)=(-1)^{k_{v}}$ for $v \mid \infty$. A Hilbert modular form of weight $k$, level $K_{1}(N)$ and character $\psi$ is a smooth function

$$
f: \boldsymbol{G L}_{2}(F) \backslash \boldsymbol{G} \boldsymbol{L}_{2}\left(\boldsymbol{A}_{F}\right) \rightarrow \mathbb{C}
$$

of moderate growth ${ }^{11}$ satisfying ${ }^{12}$

$$
f\left(\left(\begin{array}{ll}
z & \\
& z
\end{array}\right) g\left(\begin{array}{ll}
a & b \\
c & d
\end{array}\right) r(\theta)\right)=\psi(z) \psi_{N}(a) \boldsymbol{e}_{\infty}(k \cdot \theta) f(g)
$$

for each $z \in F_{\boldsymbol{A}}^{\times},\left(\begin{array}{ll}a & b \\ c & d\end{array}\right) \in K_{0}(N)$ and $\theta=\left(\theta_{v}\right)_{v \mid \infty} \in F_{\infty}$, with $r(\theta)=\prod_{v \mid \infty} r\left(\theta_{v}\right)$ and

$$
r\left(\theta_{v}\right)=\left(\begin{array}{rr}
\cos \theta_{v} & \sin \theta_{v} \\
-\sin \theta_{v} & \cos \theta_{v}
\end{array}\right) \in \boldsymbol{S O}_{2}\left(F_{v}\right) .
$$

If $k$ is constant, we say that $f$ has parallel weight; in this work, we will be almost exclusively concerned with forms of parallel weight, and we will assume that we are in this situation for the rest of this section.

\footnotetext{
${ }^{10}$ Results in the nonordinary case were presented in a preliminary version of this paper, assuming a suitable construction of $p$-adic $L$-functions generalising [Urban 2014]; I hope to present them in revised form in a future work.

11 That is, for every $g$, the function $\boldsymbol{A}^{\times} \ni y \mapsto f\left(\left(\begin{array}{l}y \\ { }_{1}\end{array}\right) g\right)$ grows at most polynomially in $|y|$ as $|y| \rightarrow \infty$.

${ }^{12}$ Recall the notation $\psi_{N}=\prod_{v \mid N} \psi_{v}$.
} 
We call $f$ holomorphic if, for each $x^{\infty} \in A^{\infty}$ and $y^{\infty} \in F_{A^{\infty}}^{\times}$, the function on $\mathfrak{H}^{\mathrm{Hom}(F, \overline{\mathbb{Q}})}=\left\{x_{\infty}+i y_{\infty} \in F \otimes \mathbb{C} \mid y_{\infty}>0\right\}$

$$
x_{\infty}+i y_{\infty} \mapsto \psi^{-1}(y)|y|^{-k / 2} f\left(\begin{array}{rr}
y & x \\
& 1
\end{array}\right)
$$

is holomorphic; in this case, such function determines $f$.

Petersson inner product. We define a Haar measure $d g$ on $Z\left(\boldsymbol{A}_{F}\right) \backslash \boldsymbol{G L}_{2}\left(\boldsymbol{A}_{F}\right)$ (where $Z \cong \boldsymbol{G}_{m}$ denotes the centre of $\boldsymbol{G} \boldsymbol{L}_{2}$ ) as follows. Recall the Iwasawa decomposition

$$
\boldsymbol{G L}_{2}\left(\boldsymbol{A}_{F}\right)=B\left(\boldsymbol{A}_{F}\right) K_{0}(1) K_{\infty}
$$

where $K_{\infty}=\prod_{v \mid \infty} \boldsymbol{S O}_{2}\left(F_{v}\right)$. Let $d k=\bigotimes_{v} d k_{v}$ be the Haar measure on $K=$ $K_{0}(1) K_{\infty}$ with volume 1 on each component. Let $d x=\bigotimes_{v} d x_{v}$ be the Haar measure such that $d x_{v}$ is the usual Lebesgue measure on $\mathbb{R}$ if $v \mid \infty$ and $\mathrm{O}_{F, v}$ has volume 1 if $v \nmid \infty$. Finally let $d^{\times} x=\bigotimes_{v} d^{\times} x_{v}$ on $F_{A}^{\times}$be the product of the measures given by $d^{\times} x_{v}=\left|d x_{v} / x_{v}\right|$ if $v \mid \infty$ and by the condition that $\mathcal{O}_{F, v}^{\times}$has volume 1 if $v \mid \infty$. Then we can use the Iwasawa decomposition $g=\left(\begin{array}{c}z \\ z\end{array}\right)\left(\begin{array}{c}y \dot{x} \\ 1\end{array}\right) k$ to define

$$
\int_{Z(\boldsymbol{A}) \backslash \boldsymbol{G} \boldsymbol{L}_{2}(\boldsymbol{A})} f(g) d g=\int_{F_{A}^{\times}} \int_{\boldsymbol{A}} \int_{K} f\left(\left(\begin{array}{rr}
y & x \\
& 1
\end{array}\right) k\right) d k d x \frac{d^{\times} y}{|y|} .
$$

The Petersson inner product of two forms $f_{1}$ and $f_{2}$ on $\mathrm{GL}_{2}(F) \backslash \mathrm{GL}_{2}(\boldsymbol{A})$ such that $f_{1} f_{2}$ is invariant under $Z(\boldsymbol{A})$ is defined by

$$
\left\langle f_{1}, f_{2}\right\rangle_{\mathrm{Pet}}=\int_{Z(\boldsymbol{A}) \backslash \boldsymbol{G} \boldsymbol{L}_{2}(\boldsymbol{A})} \overline{f_{1}(g)} f_{2}(g) d g
$$

whenever this converges (this is ensured if either $f_{1}$ or $f_{2}$ is a cuspform as defined below). It will be convenient to introduce a level-specific inner product on forms $f$ and $g$ of level $N$ :

$$
\langle f, g\rangle_{N}=\frac{\langle f, g\rangle_{\text {Pet }}}{\mu(N)}
$$

where $\mu(N)$ is the measure of $K_{0}(N)$.

1.2. Fourier expansion. Let $f$ be a (not necessarily holomorphic) Hilbert modular form. We can expand it as

$$
f(g)=C_{f}(g)+\sum_{\alpha \in F^{\times}} W_{f}\left(\left(\begin{array}{ll}
\alpha & \\
& 1
\end{array}\right) g\right)
$$


where

$$
\begin{aligned}
& C_{f}(g)=D_{F}^{-1 / 2} \int_{A / F} f\left(\left(\begin{array}{rr}
1 & x \\
& 1
\end{array}\right) g\right) d x \\
& W_{f}(g)=D_{F}^{-1 / 2} \int_{A / F} f\left(\left(\begin{array}{rr}
1 & x \\
& 1
\end{array}\right) g\right) \boldsymbol{e}(-x) d x
\end{aligned}
$$

are called the constant term and the Whittaker function of $f$, respectively. The form $f$ is called cuspidal if its constant term $C_{f}$ is identically zero. The functions of $y$ obtained by restricting the constant term and the Whittaker function to the elements $\left(\begin{array}{c}y \\ { }_{1}\end{array}\right)$ are called the Whittaker coefficients of $f$. When $f$ is holomorphic, they vanish unless $y_{\infty}>0$ and otherwise have the simple form

$$
\begin{aligned}
C_{f}\left(\begin{array}{rr}
y & x \\
& 1
\end{array}\right) & =\tilde{a}^{0}(f, y)=\psi(y)|y|^{k / 2} a(f, 0), \\
W_{f}\left(\begin{array}{rr}
y & x \\
& 1
\end{array}\right) & =\tilde{a}(f, y) \boldsymbol{e}_{\infty}\left(i y_{\infty}\right) \boldsymbol{e}(x)=\psi(y)|y|^{k / 2} a\left(f, y^{\infty} d_{F}\right) \boldsymbol{e}_{\infty}\left(i y_{\infty}\right) \boldsymbol{e}(x)
\end{aligned}
$$

for functions $\tilde{a}^{0}(f, y)$ and $\tilde{a}(f, y)$ of $y \in F_{A}^{\infty, x}$, which we call the WhittakerFourier coefficients of $f$, and a function $a(f, m)$ of the fractional ideals $m$ of $F$ that vanishes on nonintegral ideals whose values are called the Fourier coefficients of $f$.

For any $\mathbb{Z}$-submodule $A$ of $\mathbb{C}$, we denote by $M_{k}\left(K_{1}(N), \psi, A\right)$ the space of holomorphic Hilbert modular forms with Fourier coefficients in $A$ of weight $k$, level $K_{1}(N)$ and character $\psi$ and by $S_{k}\left(K_{1}(N), \psi, A\right)$ its subspace of cuspidal forms. When the character $\psi$ is trivial, we denote those spaces simply by $M_{k}\left(K_{0}(N), A\right)$ and $S_{k}\left(K_{0}(N), A\right)$, whereas linear combinations of forms of level $K_{1}(N)$ with different characters form the space $M_{k}\left(K_{1}(N), A\right)$. The notion of Whittaker-Fourier coefficients extends by linearity to the spaces $M_{k}\left(K_{1}(N), \mathbb{C}\right)$.

We can allow more general coefficients: if $A$ is a $\mathbb{Z}[1 / N]$-algebra, we define $S_{k}\left(K_{0}(N), A\right)=S_{k}\left(K_{0}(N), \mathbb{Z}[1 / N]\right) \otimes A$; this is well-defined thanks to the $q$ expansion principle [Andreatta and Goren 2005].

1.3. p-adic modular forms. Let $N$ and $P$ be coprime ideals of $O_{F}$ and $\psi$ a character of conductor dividing $N$. If $f$ is a holomorphic form of weight $k$, level $K_{1}(N P)$ and prime-to- $P$ character $\psi$ (that is, $f$ is a linear combination of forms of level $N P$ and character $\psi \psi^{\prime}$ with $\psi^{\prime}$ a character of conductor dividing $P$ ), we associate to it the formal q-expansion coefficients

$$
a_{p}\left(f, y^{\infty}\right)=\psi^{-1}(y)|y|^{-k / 2} \tilde{a}(f, y) .
$$

If $\psi^{\prime}$ is trivial, we set $a_{p}(f, m)=a_{p}\left(f, y^{\infty}\right)$ if $m$ is the ideal $m=y^{\infty} d_{F}$. 
Let $N$ be an ideal prime to $p$ and $\psi$ a character of level dividing $N$. Consider the space of classical modular forms $M_{k}\left(K_{1}\left(N p^{\infty}\right), \overline{\mathbb{Q}}\right)$ with character whose primeto- $p$ part is equal to $\psi$, and endow it with the norm given by the maximum of the $p$-adic absolute values (for the chosen embedding $\overline{\mathbb{Q}} \hookrightarrow \mathbb{C}_{p}$ ) of the Fourier coefficients. Its completion

$$
\boldsymbol{M}_{k}\left(K_{1}(N), \psi, \mathbb{C}_{p}\right)
$$

of this space is a $p$-adic Banach space called the space of $p$-adic modular forms of weight $k$, tame level $K_{1}(N)$ and tame character $\psi$. We view $M_{k}\left(K_{1}\left(N p^{r}, \psi \psi^{\prime}, \mathscr{A}\right)\right.$ (for any character $\psi^{\prime}$ of conductor divisible only by primes above $p$ ) as a subset of $\boldsymbol{M}_{k}\left(K_{1}(N), \psi, \mathscr{A}\right)$ via the $q$-expansion map.

If $\mathscr{A}$ is a complete $\mathbb{Z}_{p}$-submodule of $\mathbb{C}_{p}$, we also write $\boldsymbol{M}_{k}\left(K_{1}(N), \psi, \mathscr{A}\right)$ with obvious meaning and $\boldsymbol{S}_{k}\left(K_{1}(N), \psi, \mathscr{A}\right)$ or $\boldsymbol{S}_{k}\left(K_{0}(N), \mathscr{A}\right)$ (in the case of trivial tame character) for cuspforms; when $k=2$, we simply write

$$
S_{N}(\mathscr{A})=S_{2}\left(K_{0}(N), \mathscr{A}\right)
$$

or just $S_{N}$ if $\mathscr{A}=\mathbb{Q}_{p}$ or $\mathscr{A}=\mathbb{C}_{p}$ (as understood from context).

1.4. Operators acting on modular forms. There is a natural action of the group algebra $\mathbb{Q}\left[\boldsymbol{G L} \boldsymbol{L}_{2}\left(\boldsymbol{A}^{\infty}\right)\right]$ on modular forms induced by right translation. Here we describe several interesting operators arising from this action.

Let $m$ be an ideal of $\widehat{O}_{F}$ and $\pi_{m} \in F_{A^{\infty}}^{\times}$a generator of $m \hat{\mathcal{O}}_{F}$ that is trivial at places not dividing $m$.

The operator $[m]: M_{k}\left(K_{1}(N), \psi\right) \rightarrow M_{k}\left(K_{1}(N m), \psi\right)$ is defined by

$$
[m] f(g)=N(m)^{-k / 2} f\left(g\left(\begin{array}{ll}
1 & \\
& \pi_{m}
\end{array}\right)\right) .
$$

It acts on Fourier coefficients by

$$
a([m] f, n)=a\left(f, m^{-1} n\right) .
$$

If $\chi$ is a Hecke character of $F$, we denote by $f \mid \chi$ the form with coefficients

$$
a(f \mid \chi, n)=\chi(n) a(f, n) .
$$

For any double coset decomposition

$$
K_{1}(N)\left(\begin{array}{cc}
\pi_{m} & \\
& 1
\end{array}\right) K_{1}(N)=\coprod_{i} \gamma_{i} K_{1}(N),
$$

the Hecke operator $T(m)$ is defined by the following level-preserving action on forms $f$ in $M_{k}\left(K_{1}(N)\right)$ :

$$
T(m) f(g)=N(m)^{k / 2-1} \sum_{i} f\left(g \gamma_{i}\right) .
$$


For $m$ prime to $N$, its effect on Fourier coefficients of forms with trivial character is described by

$$
a(T(m) f, n)=\sum_{d \mid(m, n)} N(d)^{k / 2-1} a\left(f, m n / d^{2}\right) .
$$

When $m$ divides $N$, we can pick as double coset representatives the matrices

$$
\gamma_{i}=\left(\begin{array}{cc}
\pi_{m} & c_{i} \\
& 1
\end{array}\right)
$$

for $\left\{c_{i}\right\} \subset \hat{O}_{F}$ a set of representatives for $\hat{O}_{F} / m \hat{O}_{F}$. Then the operator $T(m)$ is more commonly denoted $U(m)$, and we will usually follow this practice. It acts on Fourier coefficients of forms with trivial character by

$$
a(U(m) f, n)=N(m)^{k / 2-1} a(f, m n) .
$$

Let $\boldsymbol{T}_{N}$ be the (commutative) subring of End $S_{2}\left(K_{0}(N), \mathbb{Z}\right)$ generated by the $T(m)$ for $m$ prime to $N$. A form $f$ that is an eigenfunction of all the operators in $\boldsymbol{T}_{N}$ is called a Hecke eigenform. It is called a primitive form if moreover it is normalised by $a(f, 1)=1$ and it is a newform (see Section 1.5 below for the definition) of some level dividing $N$.

As usual [Perrin-Riou 1987, Lemme 1.10], we will need the following wellknown lemma to ensure the modularity of certain generating functions:

Lemma 1.4.1. Let $A$ be a $\mathbb{Q}$-algebra. For each linear form

$$
a: \boldsymbol{T}_{N} \rightarrow A,
$$

there is a unique modular form in $\bigoplus_{N^{\prime} \mid N} S_{k}^{\text {new }}\left(K_{0}\left(N^{\prime}\right), A\right)$ whose Fourier coefficients are given by $a(T(m))$ for all $m$ prime to $N$.

Proof. In [Zhang 2001a, Corollary 3.18], the result is stated and proved when $A=\mathbb{C}$ as a consequence of the existence of a pairing $(T, f) \mapsto a_{1}(T f)$ between $\boldsymbol{T}_{N}$ and the space of modular forms of interest. But this pairing is defined over $\mathbb{Q}$; hence, the result is true for $A=\mathbb{Q}$ and by extending scalars for any $\mathbb{Q}$-algebra $A$.

Atkin-Lehner theory. For any nonzero ideal $M$ of $\mathfrak{O}_{F}$, let $W_{M} \in \boldsymbol{G L}_{2}\left(\boldsymbol{A}^{\infty}\right)$ be a matrix with components

$$
W_{M, v}=\left(\begin{array}{ll} 
& 1 \\
-\pi_{v}^{v(M)} &
\end{array}\right) \quad \text { if } v \mid M, \quad W_{M, v}=\left(\begin{array}{ll}
1 & \\
& 1
\end{array}\right) \quad \text { if } v \nmid M
$$

where $\pi_{v}$ is a uniformiser at $v$. We denote by the same name $W_{M}$ the operator acting on modular forms of level $N$ and trivial character by

$$
W_{M} f(g)=f\left(g W_{M}\right)
$$


it is self-adjoint for the Petersson inner product, and when $M$ is prime to $N$, it is proportional to the operator $[M]$ of (1.4.1). On the other hand when $M$ equals $N$, or more generally $M$ divides $N$ and is coprime to $N M^{-1}$, the operator $W_{M}$ is an involution and its action is particularly interesting. In this case, extending the definition to forms of level $K_{1}(N)$ and character ${ }^{13} \psi=\psi_{(M)} \psi_{\left(N M^{-1}\right)}$ with $\psi_{(C)}$ of conductor dividing $C$, we have

$$
W_{M} f(g)=\psi_{(M)}^{-1}(\operatorname{det} g) \psi_{(M)}^{-1}\left(\pi_{M}\right) f\left(g W_{M}\right)
$$

where $\pi_{M}$ is the idele with nontrivial components only at $v \mid M$ and given there by $\pi_{v}^{v(M)}$. It is easy to check that this definition is independent of the choice of uniformisers. The effect of the $W_{M}$-action on newforms is described by AtkinLehner theory; we summarise it here (in the case $M=N$ ), referring to [Casselman 1973] for the details.

Let $\pi$ be an irreducible infinite-dimensional automorphic representation of $\boldsymbol{G} \boldsymbol{L}_{2}\left(\boldsymbol{A}_{F}\right)$ of central character $\psi$. Up to scaling, there is a unique newform $f$ in the space of $\pi$. It is characterised by either equivalent property: it is fixed by a subgroup $K_{1}(N)$ with $N$ minimal among the $N^{\prime}$ for which $\pi^{K_{1}\left(N^{\prime}\right)} \neq 0$ or its Mellin transform is (a multiple of) the $L$-function $L(\pi, s)$ of $\pi$. In the case of a holomorphic cuspform, this is equivalent to requiring that it belongs to the space of newforms defined in Section 1.5 below. There is a functional equation relating the $L$-function $L(s, \pi)$ of $\pi$ and the $L$-function $L(1-s, \tilde{\pi})$ of the contragredient representation; as $\tilde{\pi} \cong \psi^{-1} \cdot \bar{\pi}$, it translates into the following description of the action of $W_{N}$ on newforms. Suppose that the eigenform $f \in S_{k}\left(K_{1}(N), \psi\right)$ is a newform in the representation $\pi$ it generates; then we have

$$
W_{N} f(g)=(-i)^{[F: \mathbb{Q}] k} \tau(f) f^{\rho}(g)
$$

where $f^{\rho}$ is the form with coefficients

$$
a\left(f^{\rho}, m\right)=\overline{a(f, m)}
$$

and $\tau(f)=\tau(\pi)$ is an algebraic number of complex absolute value 1 .

Trace of a modular form. The trace of a modular form $f$ of level $N D$ and trivial character is the form of level $N$

$$
\operatorname{Tr}_{N D / N}(f)(g)=\sum_{\gamma \in K_{0}(N) / K_{0}(N D)} f(g \gamma) .
$$

${ }^{13}$ Notice that a decomposition of $\psi$ as described is only unique up to class group characters (that is, Hecke characters of level 1). We will only be using the operator $W_{M}$ for $M$ a proper divisor of $N$ in a case in which a decomposition is naturally given. 
It is the adjoint of inclusion of forms of level $N$ for the rescaled Petersson product:

$$
\left\langle f, \operatorname{Tr}_{N D / N} g\right\rangle_{N}=\langle f, g\rangle_{N D}
$$

if $f$ has level $N$ and $g$ has level $D$.

Suppose that $D$ is squarefree and prime to $N$, in which case we can write $\operatorname{Tr}_{D}=\operatorname{Tr}_{N D / N}$ without risk of ambiguity. A set of coset representatives for $K_{0}(N) / K_{0}(N D)$ is given by elements $\gamma_{j, \delta}$ for $\delta \mid D, j \in \mathscr{O}_{F, v} / \delta \mathscr{O}_{F, v}$, having components

$$
\gamma_{j, \delta, v}=\left(\begin{array}{ll}
1 & j \\
& 1
\end{array}\right)\left(\begin{array}{cc} 
& 1 \\
-1 &
\end{array}\right)=\frac{1}{\pi_{v}}\left(\begin{array}{ll}
\pi_{v} & j \\
& 1
\end{array}\right)\left(\begin{array}{cc} 
& 1 \\
-\pi_{v} &
\end{array}\right)
$$

at places $v \mid \delta$ and $\gamma_{j, \delta, v}=1$ everywhere else. From the second decomposition given just above, if $f$ has weight 2 , we obtain

$$
a\left(\operatorname{Tr}_{D}(f), m\right)=\sum_{\delta \mid D} a\left(U(\delta) f^{(\delta)}, m\right)=\sum_{\delta \mid D} a\left(f^{\delta)}, m \delta\right)
$$

where $f^{(\delta)}(g)=f\left(g W_{\delta}\right)$ with $W_{\delta}$ as in (1.4.2).

Remark 1.4.2. If $D$ is prime to $p$, the various trace operators $\operatorname{Tr}_{N D p^{r} / N p^{r}}$ extend to a continuous operator $\operatorname{Tr}_{N D / N}$ on $p$-adic modular forms of tame level $N D$. Similarly, the operators $[m], T(m)$ and $W_{m}$ for $m$ prime to $N p$ extend to continuous operators on $p$-adic modular forms of tame level $N$.

Ordinary projector. Let $L$ be a complete subfield of $\mathbb{C}_{p}$. Following Hida (see, e.g., [Hida 1991, §3]), we can define for each $\wp \mid p$ an idempotent

$$
e_{\wp}=\lim _{n \rightarrow \infty} U_{\wp}^{n !}: S_{N}(L) \rightarrow S_{N_{\wp}}(L)
$$

that is surjective onto $S_{N_{\wp}}^{\wp-\text { ord }}(L)$, the subspace of $S_{N_{\wp}}(L)$ spanned by $U_{\wp}$-eigenforms with unit eigenvalue.

Let $P=\prod_{\wp \mid p} \wp$. Then we similarly have a surjective idempotent

$$
e=\prod_{\wp \mid p} e_{\wp}: S_{N}(L) \rightarrow S_{N P}^{\mathrm{ord}}(L),
$$

where $S_{N P}^{\text {ord }}(L)$ is the subspace of $S_{N P}(L)$ spanned by simultaneous $U_{\wp}$-eigenforms with unit eigenvalue.

1.5. Fourier coefficients of old forms. As we will study modular forms through their Fourier coefficients, we give here a criterion for recognising the coefficients of certain old forms. ${ }^{14}$ Let $N$ and $P$ be coprime ideals of $O_{F}$. The space $S_{N P}^{N \text {-old }} \subset S_{N P}$ is the space spanned by forms $f=[d] f^{\prime}$ for some $1 \neq d \mid N$ and some cuspform $f^{\prime}$

\footnotetext{
${ }^{14}$ See [Zhang 2001a, §4.4.4].
} 
of level $N^{\prime} P$ with $N^{\prime} \mid d^{-1} N$. In the case $P=1$, we define the space of newforms of level dividing $N$ to be orthogonal to the space of old forms for the Petersson inner product. We denote by $S_{N}^{\text {old }} \subset S_{N}$ the closed subspace generated by the image of $S_{N p^{\infty}}^{N \text {-old }}$ in $S_{N}$. (The coefficient ring will always be either a finite extension of $\mathbb{Q}_{p}$ or $\mathbb{C}_{p}$ as understood from context when not present explicitly in the notation.)

Let now $\mathscr{Y}$ be the space of functions $f: N_{F} \rightarrow \mathscr{A}$ modulo those for which there is an ideal $M$ prime to $p$ such that $f(n)=0$ for all $n$ prime to $M$. A function $f \in \mathscr{Y}$ is called multiplicative if it satisfies ${ }^{15} f(m n)=f(m) f(n)$ for all $(m, n)=1$. For $h$ a multiplicative function, a function $f$ is called an $h$-derivative if it satisfies $f(m n)=h(m) f(n)+h(n) f(m)$ for all $(m, n)=1$.

Let $\sigma_{1}$ and $r$ be the multiplicative elements of $\mathscr{S}$ defined by

$$
\sigma_{1}(m)=\sum_{d \mid m} N(d), \quad r(m)=\sum_{d \mid m} \varepsilon_{E / F}(d)
$$

(where $E$ is a totally imaginary quadratic extension of $F$ of discriminant prime to $p) .{ }^{16}$ Let $P=\prod_{\wp \mid p} \wp \subset \mathcal{O}_{F}$. We define a subspace $\mathscr{D}_{N} \subset \mathscr{Y}$ to be generated by $\sigma_{1}, r, \sigma_{1}$-derivatives, $r$-derivatives and Fourier coefficients of forms in $S_{N P}^{N \text {-old }}$.

Lemma 1.5.1. The q-expansion map $S_{N P}^{\text {ord }} / S_{N P}^{N-o l d} \rightarrow \mathscr{S} / \mathscr{D}_{N}$ is injective.

Proof. First notice that it is enough to show this when the coefficient ring is a number field $L$ over which $S_{N P}^{\text {ord }}$ is defined (it suffices for $L$ to contain all the eigenvalues of the operators $T_{\ell}(\ell \nmid N p)$ and $U_{\wp}$ on $\left.S_{N P}(L)\right)$. By [Zhang 2001a, Proposition 4.5.1], the kernel of $S_{N P}(L) / S_{N P}^{N \text {-old }}(L) \rightarrow \mathscr{S} / \mathscr{D}_{N}$ is at most generated by $S_{N P}^{p \text {-old }}(L)=\sum_{\wp \mid p} S_{N P}^{\wp \text {-old }}(L)$, the space of forms that are old at some $\wp \mid p$. To conclude, it suffices to show that for each $\wp \mid p$ we have $I:=S_{N P}^{\wp \text {-old }} \cap S_{N P}^{\wp \text {-ord }}=0$. The intersection $I$ is stable under the action of $\boldsymbol{T}_{N P}$, which decomposes it into spaces $I\left[f_{i}\right] \subset S_{N P}\left[f_{i}\right]$ corresponding to eigenforms $f_{i}$ of level $N^{\prime}$ or $N^{\prime} \wp$ for some $N^{\prime} \mid N P \wp^{-1}$. If $f_{i}$ has level $N^{\prime} \wp$, then $S_{N P}\left[f_{i}\right]$ does not contain any nonzero $\wp$-oldforms. If $f_{i}$ has level $N^{\prime}$ with $\wp \nmid N^{\prime}$, then $S_{N P}^{\text {ord }}\left[f_{i}\right]$ is either zero or the line spanned by the ordinary $\wp$-stabilisation of $f_{i}$, whereas $S_{N P}^{\wp \text {-old }}\left[f_{i}\right]$ is the line spanned by $[\wp] f_{i}$. We conclude that $I\left[f_{i}\right]=0$ in all cases.

Remark 1.5.2. The operators $U_{\wp}$ for $\wp \mid p$ extend to operators on $\mathscr{S}$ via $U_{\wp} f(m)=$ $f(m \wp)$. The Hecke algebra $\boldsymbol{T}_{N p}$ acts on the image $\mathscr{Y}_{N}$ of $\boldsymbol{S}_{N}$ in $\mathscr{S} / \mathscr{D}_{N}$.

1.6. The functional $\boldsymbol{l}_{f_{\alpha}}$. Recall from the introduction that we have fixed an ordinary primitive Hilbert modular newform $f$ of level $K_{0}(N)$. If $\alpha_{\wp}$ is the unit root of

${ }^{15}$ This relation and the following are of course to be understood to hold in $\mathscr{S}$.

${ }^{16} \mathrm{We}$ will see below that $\sigma_{1}$ and $r$ are the Fourier coefficients of weight-1 Eisenstein series and theta series. 
the $\wp$-th Hecke polynomial of $f, \beta_{\wp}$ is the other root and the operator [ø] is as in (1.4.1), then the $p$-stabilisation of $f$ is

$$
f_{\alpha}=\prod_{\wp \mid p}\left(1-\beta_{\wp}[\wp]\right) f
$$

a form of level $K_{0}\left(N \prod_{\wp \mid p} \wp\right)$ satisfying $U_{\wp} f_{\alpha}=\alpha_{\wp} f_{\alpha}$ for all $\wp \mid p$.

We define a functional, first introduced by Hida, that plays the role of projection onto the $f$-component. Both sides of our main formula will be images of $p$-adic modular forms under this operator.

Let $P$ be an ideal of $O_{F}$ divisible exactly by the primes $\wp \mid p$. For a form $g \in M_{2}\left(K_{0}(N P)\right)$ with $r \geq 1$, let

$$
l_{f_{\alpha}}(g)=\frac{\left\langle W_{N P} f_{\alpha}^{\rho}, g\right\rangle}{\left\langle W_{N P} f_{\alpha}^{\rho}, f_{\alpha}\right\rangle} .
$$

Let $L \subset \overline{\mathbb{Q}}_{p}$ be the extension of $\mathbb{Q}_{p}$ generated by $a(f, m)$ for all ideals $m$ and $\alpha_{\wp}$ for $\wp \mid p$.

Lemma 1.6.1 (Hida). The above formula defines a linear functional

$$
l_{f_{\alpha}}: M_{2}\left(K_{0}\left(N p^{\infty}\right), L\right) \rightarrow L
$$

satisfying:

(1) $\mathrm{On} \mathrm{M}_{2}\left(K_{0}(N), L\right)$, we have

$$
l_{f_{\alpha}}=\prod_{\wp \mid p}\left(1-\frac{N \wp}{\alpha_{\wp}^{2}}\right)^{-1} \mathbb{1}_{f}
$$

where $\mathbb{1}_{f}(g)=\langle f, g\rangle /\langle f, f\rangle$.

(2) On $M_{2}\left(K_{0}\left(N \wp^{r}\right)\right)$, we have, for each nonnegative $t \leq r-1$,

$$
l_{f_{\alpha}} \circ U_{\wp}^{t}=\alpha_{\wp}(f)^{t} l_{f_{\alpha}} .
$$

(3) If each $\iota_{p}\left(\alpha_{\wp}\right)$ is a p-adic unit, $l_{f_{\alpha}}$ admits a continuous extension to $p$-adic modular forms still denoted

$$
l_{f_{\alpha}}: \boldsymbol{M}_{N}(L) \rightarrow L
$$

Proof. See [Hida 1991, Lemma 9.3], where the well-definedness of the functional and its extension to $p$-adic modular forms are proved more generally for Hida families. For part (1), the computation is the same as in the case of elliptic modular forms: see [Perrin-Riou 1988] or [Hida 1985, §4]. 
Some quotient spaces. Let $\overline{\mathscr{S}}=\mathscr{Y} / \mathscr{D}_{N}$. The ordinary projection operator $e$ is not defined on all arithmetic functions; however, its $\operatorname{kernel} \operatorname{Ker}(e)$ is a well-defined subspace of $\mathscr{Y}$. We define

$$
\overline{\mathscr{S}}^{\text {ord }}:=\mathscr{S} / \mathscr{D}_{N}+\operatorname{Ker}(e) .
$$

The quotient map $\overline{\mathscr{S}} \rightarrow \overline{\mathscr{S}}^{\text {ord }}$ is clearly injective when restricted to the image of $S_{N P}^{\text {ord }}$, where $P=\prod_{\wp \mid p} \wp$. Then we denote by $\overline{\mathscr{Y}}_{N}^{\text {ord }}$ the image of $S_{N P}^{\text {ord }}$ in either $\overline{\mathscr{Y}}$ or $\overline{\mathscr{Y}}$ ord It is also identified with the common image of $S_{N P}$ and $S_{N}$ in $\overline{\mathscr{S}}^{\text {ord }}$. We denote by $\bar{\varphi}_{N}^{p \text {-adic }} \subset \overline{\mathscr{Y}}$ the image of $\boldsymbol{S}_{N}$.

We obtain a commutative diagram (where $L$ is as usual any sufficiently large finite extension of $\mathbb{Q}_{p}$ ):

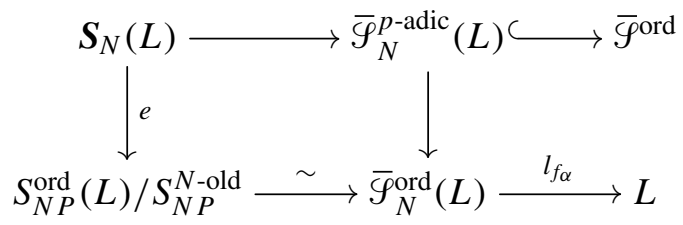

where the right-hand vertical map is the restriction of the quotient $\overline{\mathscr{S}} \rightarrow \overline{\mathscr{S}}$ ord and the bottom horizontal map is an isomorphism by Lemma 1.5.1.

\section{Theta measure}

We construct a measure on the Galois group of the maximal abelian extension of $E$ unramified outside $p$ with values in $p$-adic theta series and compute its Fourier expansion.

2.1. Weil representation. We first define the Weil representation. See [Bump 1997, $\S 4.8$ ] for an introduction and [Waldspurger 1985] or [Yuan et al. 2013] for our conventions on the representation for similitude groups.

Local setting. Let $V=(V, q)$ be a quadratic space over a local field $F$ of characteristic not 2 with a quadratic form $q$; we choose a nontrivial additive character $\boldsymbol{e}$ of $F$. For simplicity, we assume $V$ has even dimension. For $u \in F^{\times}$, we denote by $V_{u}$ the quadratic space $(V, u q)$. We let $\boldsymbol{G L}_{2}(F) \times \boldsymbol{G} \boldsymbol{O}(V)$ act on the space $\mathscr{S}\left(V \times F^{\times}\right)$ of Schwartz functions as follows (here $v: \boldsymbol{G} \boldsymbol{O}(V) \rightarrow \boldsymbol{G}_{m}$ denotes the similitude character):

- $r(h) \phi(t, u)=\phi\left(h^{-1} t, v(h) u\right)$ for $h \in \boldsymbol{G O}(V)$,

- $r(n(x)) \phi(t, u)=\boldsymbol{e}(x u q(t)) \phi(t, u)$ for $n(x)=\left(\begin{array}{r}1 x \\ 1\end{array}\right) \in \boldsymbol{G L}_{2}$,

- $r\left({ }^{a}{ }_{d}\right) \phi(t, u)=\chi_{V}(a)|a / d|^{(\operatorname{dim} V) / 4} \phi\left(a t, d^{-1} a^{-1} u\right)$,

- $r(w) \phi(x, u)=\gamma\left(V_{u}\right) \hat{\phi}(x, u)$ for $w=\left({ }_{-1}^{1}\right)$. 
Here $\chi_{V}$ is the quadratic character associated with $V, \gamma\left(V_{u}\right)$ is a certain square root of $\chi(-1)$ and $\hat{\phi}$ denotes the Fourier transform in the first variable

$$
\hat{\phi}(x, u)=\int_{V} \phi(y, u) \boldsymbol{e}(-u\langle x, y\rangle) d y
$$

where $\langle\cdot, \cdot\rangle$ is the bilinear form associated with $q$ and $d y$ is the self-dual Haar measure.

Global setting. Given a quadratic space $(V, q)$ over a global field $F$ of characteristic not 2 (and a nontrivial additive character $\boldsymbol{e}: F \backslash \boldsymbol{A}_{F} \rightarrow \mathbb{C}^{\times}$), the Weil representation is the restricted tensor product $r$ of the associated local Weil representations, with spherical functions $\phi_{v}(t, u)=\mathbb{1}_{\mathscr{V}_{v} \times \mathbb{O}_{F, v}^{\times}}(x, u)$ for some choice of lattices $\mathscr{V}_{v} \subset V\left(F_{v}\right)$.

The case of interest to us is the following: $F$ is a totally real number field, $V=(E, \mathfrak{N})$ is given by a quadratic $\mathrm{CM}$ extension $E / F$ with the norm form $\mathfrak{N}=N_{E / F}$ and the lattices $\mathscr{O}_{E, v} \subset E_{v}$ and the additive character $\boldsymbol{e}$ is the standard one. We denote $G=\boldsymbol{G} \boldsymbol{L}_{2}$ and $H=\boldsymbol{G} \boldsymbol{O}(V)$, two algebraic groups defined over $F$; we have $H \cong \operatorname{Res}_{E / F} \boldsymbol{G}_{m}$. In this case, we have

$$
\chi_{V}=\varepsilon_{E / F}=\varepsilon,
$$

where $\varepsilon_{E / F}$ is the quadratic character of $F_{A}^{\times}$associated with the extension $E / F$. The self-dual measure on $E_{v}$ is the one giving $\mathscr{O}_{E, v}$ volume $\left|\mathscr{O}_{E, v} / \mathfrak{D}_{v}\right|^{-1 / 2}$ where $\mathfrak{D}_{v}$ is the relative different. Moreover, the constant $\gamma$ can be explicitly described [Bushnell and Henniart 2006, §38.6, §30.4, 23.5$]$ : in the case $v \mid \Delta_{E / F}$, which is the only one we will be using, such description is in terms of a local Gauss sum $\kappa(v)$ :

$$
\gamma\left(E_{v}, u \mathfrak{N}\right)=\varepsilon_{v}(u) \kappa(v)=\varepsilon_{v}(u)\left|\pi_{v}\right|^{1 / 2} \sum_{x \in\left(\mathscr{O}_{F, v} / \pi_{v} \mathbb{O}_{F, v}\right)^{\times}} \varepsilon\left(x / \pi_{v}\right) \boldsymbol{e}_{v}\left(x / \pi_{v}\right) .
$$

Notice that our $\kappa(v)$ is the inverse of the quantity denoted by the same name in [Zhang 2001a, Proposition 3.5.2].

2.2. Theta series. We define the theta kernel to be

$$
\theta_{\phi}(g, h)=\sum_{(t, u) \in V \times F^{\times}} r(g, h) \phi(t, u),
$$

an automorphic form for the group $\boldsymbol{G} \boldsymbol{L}_{2}(F) \backslash \boldsymbol{G} \boldsymbol{L}_{2}\left(\boldsymbol{A}_{F}\right) \times \boldsymbol{G O}(V) \backslash \boldsymbol{G O}\left(V_{\boldsymbol{A}_{F}}\right)$.

If $W$ is an automorphic function for $H$ that is trivial at infinity (which is the same thing as a linear combination of finite-order Hecke characters of $E$ ), we define the theta series $^{17}$

$$
\theta_{\phi}(\mathcal{W})(g)=\int_{H(F) \backslash H\left(\boldsymbol{A}_{F}\right)} \mathcal{W}\left(h^{-1}\right) \theta_{\phi}(g, h) d h,
$$

${ }^{17}$ The reason for taking $\mathcal{W}\left(h^{-1}\right)$ rather than $\mathcal{W}(h)$ is that we want $\theta_{\phi}(\mathcal{W})$ to be the series classically denoted $\Theta(W)$ for a suitable choice of $\phi$ - this will be clear from the computations below. 
which is an automorphic form on $G$. Here the measure $d h$ is the product of the measure on $H\left(\boldsymbol{A}^{\infty}\right)$ that gives volume 1 to the compact $U_{0}=\hat{\mathcal{O}}_{E}^{\times}$and any fixed measure $^{18}$ on $H\left(\boldsymbol{A}_{\infty}\right)$.

Let us explain how to explicitly compute the integral in our situation. For each open compact subgroup $U \subset H\left(\boldsymbol{A}_{F}^{\infty}\right)=E_{A^{\infty}}$, we have exact sequences

$$
1 \rightarrow \mathrm{O}_{E, U}^{\times} \backslash U E_{\infty}^{\times} \rightarrow E^{\times} \backslash E_{A}^{\times} \rightarrow E^{\times} U \backslash E_{A^{\infty}}^{\times} \rightarrow 1
$$

and

$$
1 \rightarrow \mu(U) \backslash U E_{\infty}^{1} \rightarrow \mathbb{O}_{E, U}^{\times} \backslash U E_{\infty}^{\times} \stackrel{\mathfrak{N}_{\infty}}{\longrightarrow} N\left(\mathbb{O}_{E, U}^{\times}\right) \backslash F_{\infty}^{+} \rightarrow 1 .
$$

The notation used is the following: $\mathcal{O}_{E, U}^{\times}=E^{\times} \cap U \supset \mu(U)=$ the subset of roots of unity, $\mathfrak{N}_{\infty}: E_{\infty}^{\times} \rightarrow F_{\infty}^{+}$is the norm map at the infinite places and $E_{\infty}^{1}$ is its kernel.

We can choose a splitting $\iota$ of the first sequence, for example

$$
\iota: E^{\times} U \backslash E_{A^{\infty}}^{\times} \cong E^{\times} U \backslash\left(E_{A}^{\times}\right)^{1, \|} \hookrightarrow E^{\times} \backslash E_{A}^{\times},
$$

where $\left(E_{\boldsymbol{A}}^{\times}\right)^{1, \|}$ denotes the set of idèles of adelic norm 1 with infinity component $h_{\infty}=(h, \ldots, h)$ for some real number $h>0$ and the isomorphism is the unique one that gives the identity once composed with projection onto the finite part.

We begin to expand the series, evaluating the integral as explained above and exploiting the fact that the action of $H\left(F_{\infty}\right)=E_{\infty}^{\times}$on $\phi(t, u)$ factors through the norm. We take $U$ to be small enough so that $\mathcal{W}$ and $\phi$ are invariant under $U$ and denote

$$
\bar{\phi}_{v}(t, u)=\int_{H\left(F_{v}\right)} r(h) \phi_{v}(t, u) d h \quad \text { if } v \mid \infty
$$

and $\bar{\phi}=\prod_{v \nmid \infty} \phi_{v} \prod_{v \mid \infty} \bar{\phi}_{v}$. A specific choice of $\bar{\phi}_{v}$ will be made shortly; for the moment, we just say, and use in the following computation, that we will take $u \mapsto \bar{\phi}_{v}(t, u)$ to be supported on $\mathbb{R}^{+}$.

We have

$$
\begin{aligned}
\theta_{\phi}(\mathcal{W})(g) & =\int_{E^{\times} \backslash E_{A}^{\times}} \mathcal{W}\left(h^{-1}\right) \theta_{\phi}(g, h) d h \\
& =w_{U}^{-1} \int_{U} \int_{E_{\infty}^{1}} \int_{\mathfrak{N}\left(\mathcal{O}_{E, U}^{\times}\right) \backslash F_{\infty}^{+}} \int_{E^{\times} U \backslash E_{A}^{\times}} \mathcal{W}\left(\iota(a)^{-1}\right) \\
\cdot & \sum_{(t, u) \in E \times F^{\times}} r(g, \iota(a) h) \phi(t, u) d a d h .
\end{aligned}
$$

Here $w_{U}=|\mu(U)|$ and $d h$ denotes the measure on $U \times E_{\infty}^{1} \times F_{\infty}^{+}=U \times H\left(F_{\infty}\right)$. We partially collapse the integral over $\mathfrak{N}\left(\mathcal{O}_{E, U}^{\times}\right) \backslash F_{\infty}^{+}$and the sum over $u \in F^{\times}$and

${ }^{18}$ There will be no ambiguity since later we will choose $\phi_{\infty}$ to again be any fixed Schwartz function whose integral over $H\left(\boldsymbol{A}_{\infty}\right)$ with respect to the chosen measure is a specified function $\bar{\phi}_{\infty}$. 
use our choice of $\phi_{\infty}$ to get

$$
\begin{aligned}
& =w_{U}^{-1} \operatorname{vol}(U) \int_{E^{\times} U \backslash E_{A^{\infty}}^{\times}} \mathcal{W}\left(\iota(a)^{-1}\right) \sum_{u \in \mathfrak{N}\left(\mathbb{O}_{E, U}^{\times}\right) \backslash F^{+}} \sum_{t \in E} r(g, \iota(a)) \bar{\phi}(t, u) d a \\
& =w^{-1} \frac{h}{h_{U}} \int_{E^{\times} U \backslash E_{A^{\infty}}^{\times}} \mathcal{W}\left(\iota(a)^{-1}\right) v_{U} \sum_{u \in \mathfrak{N}\left(\mathbb{O}_{E, U}^{\times}\right) \backslash F^{+}} \sum_{t \in E} r(g, \iota(a)) \bar{\phi}(t, u) d a .
\end{aligned}
$$

Here in the last step, we have defined $v_{U}=\left[\mathfrak{N}\left(\mathcal{O}_{E}^{\times}\right): \mathfrak{N}\left(\mathcal{O}_{E, U}^{\times}\right)\right]$and computed $\operatorname{vol}(U)=\operatorname{vol}\left(U_{0}\right)\left(h / h_{U}\right)\left(w_{U} / w\right) v_{U}^{-1}$, where $U_{0}=\hat{O}_{E}^{\times}, h_{U}=\left|E^{\times} U \backslash E_{A^{\infty}}^{\times}\right|, h=h_{U_{0}}$ and $w=w_{U_{0}}$. Recall that our measure satisfies $\operatorname{vol}\left(U_{0}\right)=1$. The remaining integral is just a finite sum.

The sum over $u$ is actually finite due to the integrality constraints imposed by $\phi$ at finite places. ${ }^{19}$

2.3. Theta measure. We define a measure with values in $p$-adic modular forms on the group

$$
\varphi^{\prime}=\operatorname{Gal}\left(E_{\infty}^{\prime} / E\right) \cong \lim _{\longleftarrow} \overline{E^{\times} U_{p^{n}}} \backslash E_{A^{\infty}}^{\times}
$$

where the overline denotes closure and $E_{\infty}^{\prime}$ is the maximal abelian extension of $E$ unramified outside $p$, that is, the union of the ray class fields of $E$ of $p$-power ray $U_{p^{n}}=\prod_{v}\left\{\right.$ units $\left.\equiv 1 \bmod p^{n} \mathscr{O}_{E, v}\right\}$, and the isomorphism is given by class field theory. The topology is the profinite topology.

Recall that a measure on a topological space $\mathscr{G}$ with values in a $p$-adic Banach space $\boldsymbol{M}$ is a $\mathbb{C}_{p}$-linear functional

$$
\mu: \mathscr{C}\left(\mathscr{G}, \mathbb{C}_{p}\right) \rightarrow M
$$

on continuous $\mathbb{C}_{p}$-valued functions, which is continuous (equivalently, bounded) with respect to the supremum norm on $\mathscr{C}\left(\mathscr{G}, \mathbb{C}_{p}\right)$. The linearity property will be called distributional property in what follows. The boundedness property will in each case at hand be verified on the set of $p$-adic characters of $\mathscr{G}$, which in our cases generates the whole of $\mathscr{C}\left(\mathscr{G}, \mathbb{C}_{p}\right)$ (classically, the continuity of $\mu$ goes by the name of abstract Kummer congruences for $\mu)$.

When $\boldsymbol{M}=\boldsymbol{M}_{0} \otimes_{\mathbb{Q}_{p}} \mathbb{C}_{p}$ for a $p$-adic Banach space $\boldsymbol{M}_{0}$ over $\mathbb{Q}_{p}$, the measure $\mu$ is said to be defined over $\mathbb{Q}_{p}$ if $\mu(\mathcal{W}) \in \boldsymbol{M}_{0} \otimes \mathbb{Q}_{p}(\mathcal{W})$ whenever the function $\mathscr{W}$ on $\mathscr{G}$ has values in $\mathbb{Q}_{p}(\mathcal{W}) \subset \overline{\mathbb{Q}}_{p} \subset \mathbb{C}_{p}$.

${ }^{19}$ We will see this in more detail shortly. We are also using the definition of $\bar{\phi}_{\infty}$ in order to freely replace the sum over $u \in F^{\times}$with a sum over $u \in F^{+}$—in fact, a slight variation would be necessary when $\operatorname{det} g_{\infty} \notin F_{\infty}^{+}$, but this is a situation we won't encounter. 
Definition 2.3.1. The theta measure $d \Theta$ on $\varphi^{\prime}$ is defined by

$$
\Theta(\mathcal{W})=\int_{\mathscr{G}^{\prime}} \mathscr{W}(\sigma) d \Theta(\sigma):=\theta_{\phi}(\mathcal{W}),
$$

for any function $\mathcal{W}: \mathscr{G}^{\prime} \rightarrow \overline{\mathbb{Q}}$ factoring through a finite quotient of $\mathcal{G}^{\prime}$, where the function $\phi$ is chosen as follows:

- For $v \nmid p \infty, \phi_{v}(t, u)=\mathbb{1}_{\bigcirc_{E, v}}(t) \mathbb{1}_{d_{F_{v}}^{-1, \times}}(u)$.

- For $v \mid p$,

$$
\phi_{v}(t, u)=\left[\mathcal{O}_{E, v}^{\times}: U_{v}^{\prime}\right] \mathbb{1}_{U_{v}^{\prime}}(t) \mathbb{1}_{d_{F_{v}}^{-1, \times}}(u),
$$

where $U_{v}^{\prime} \subset \mathcal{O}_{E, v}^{\times}$is any small-enough compact set - that is, $U_{v}^{\prime} \subset U_{v}$ if $\mathcal{W}$ is invariant under $U=\prod_{v} U_{v}$, and the definition does not depend on the choice of $U_{v}$. (In practice, we will choose $U_{v}^{\prime}=U_{v}$ if $U_{v}$ is maximal with respect to the property just mentioned.)

- For $v \mid \infty, \phi_{v}(t, u)$ is a Schwartz function such that

$$
\int_{H\left(F_{v}\right)} r(h) \phi_{v}(t, u) d h=\bar{\phi}_{v}(t, u)=1_{\mathbb{R}^{+}}(u) \exp (-2 \pi u N(t)) .
$$

(See [Yuan et al. 2013, §4.1] for more details on this choice.)

In Corollary 2.4 .2 below, we will show that this in fact defines a measure on $\varphi^{\prime}$ with values in $p$-adic Hilbert modular forms of weight 1 , tame level $\Delta_{E / F}$ and character $\varepsilon$.

2.4. Fourier expansion of the theta measure, I. We compute the Fourier expansion of the theta measure on $\mathscr{G}^{\prime}$, carrying on the calculation started in Section 2.2. In the case where $g=\left(\begin{array}{cc}y & x \\ 1\end{array}\right)$ with $y_{\infty}>0$, the sum over $(u, t)$ in (2.2.1) evaluates to

$$
\varepsilon(y)|y|^{1 / 2} \sum_{u, t} \phi^{\infty}\left(a^{-1} y t, \mathfrak{N}(a) y^{-1} u\right) \boldsymbol{e}_{\infty}\left(i y_{\infty} u N(t)\right) \boldsymbol{e}(x u \mathfrak{N}(t)) .
$$

Then we compute the sum of this expression over the finite quotient $\mathscr{G}_{U}^{\prime}$ of $\mathscr{G}^{\prime}$, with $\varphi_{U}^{\prime} \cong E^{\times} U \backslash E_{A^{\infty}}^{\times}$.

We assume $\mathscr{W}$ is a character so that $\mathscr{W}\left(a^{-1}\right)=\bar{W}(a)$ where $\bar{W}=W^{-1}$.

First we precompute the product of all the constants appearing in the theta series of (2.2.1), including the one from $\phi-$ we take

$$
\phi_{v}(t, u)=\left[\mathcal{O}_{E, v}^{\times}: U_{v}\right] \mathbb{1}_{U_{v}}(t) \mathbb{1}_{\mathscr{O}_{F}^{\times}}(u),
$$

so

$$
\begin{aligned}
w \frac{h}{h_{U}} v_{U}\left[\mathbb{O}_{E, v}^{\times}: U_{v}\right] & =w\left[\mathbb{O}_{E}^{\times} \backslash \hat{\mathbb{O}}_{E, v}^{\times}: \mathscr{O}_{E, U}^{\times} \backslash U\right]^{-1}\left[\mathfrak{N}\left(\mathscr{O}_{E}^{\times}\right): \mathfrak{N}\left(\mathbb{O}_{E, U}^{\times}\right)\right]^{-1}\left[\hat{\mathscr{O}}_{E}^{\times}: U\right] \\
& =w\left[\mu\left(\mathscr{O}_{E}\right): \mu(U)\right]=w_{U}^{-1} .
\end{aligned}
$$


This computation together with (2.2.1) and (2.4.1) gives

$$
\begin{gathered}
\Theta(\mathcal{W})=\varepsilon(y)|y|^{1 / 2} w_{U}^{-1} \sum_{a \in E^{\times} U \backslash E_{A^{\infty}}^{\times}} \overline{\mathscr{W}}(a) \sum_{t \in E, u \in \mathfrak{N}\left(\mathscr{O}_{E, U}^{\times}\right) \backslash F^{+}} \phi^{p \infty}\left(a^{-1} y t, \mathfrak{N}(a) y^{-1} u\right) \\
\cdot \mathbb{1}_{\mathbb{O}_{E, U, p}^{\times}}\left(a^{-1} y t\right) \mathbb{1}_{d_{F p}^{-1, \times}}\left(\mathfrak{N}(a) y^{-1} u\right) \boldsymbol{e}_{\infty}\left(i y_{\infty} u \mathfrak{N}(t)\right) \boldsymbol{e}(x u \mathfrak{N}(t)) \\
=\varepsilon(y) \bar{W}(y)|y|^{1 / 2} w_{U}^{-1} \sum_{a \in E^{\times} U \backslash E_{A^{\infty}}^{\times}} \bar{W}(a) \sum_{t \in E, u \in \mathfrak{N}\left(\mathscr{O}_{E, U}^{\times}\right) \backslash F^{+}} \mathbb{1}_{\widehat{\widehat{O}_{E, U}} \cap 0_{E, U, p}^{\times}}\left(a^{-1} t\right) \\
\cdot \mathbb{1}\left[\mathfrak{N}(a) y u \widehat{O}_{F}=d_{F}^{-1}\right] \boldsymbol{e}_{\infty}\left(i y_{\infty} u \mathfrak{N}(t)\right) \boldsymbol{e}(x u \mathfrak{N}(t)),
\end{gathered}
$$

where we have made the change of variable $a \rightarrow a y^{\infty}$.

Now we make the substitution $u \mathfrak{N}(t)=\xi$ and observe that the contribution to the $\xi$-th term is equal to 0 if $\left(\xi y d_{F}, p\right) \neq 1$ and otherwise it equals $\bar{W}(a)$ times the cardinality of the set

$$
R_{a^{-1}}(\xi, y)=\left\{(t, u) \in \mathbb{O}_{E} \times F^{+} \mid t \in U_{p}, u \mathfrak{N}(t)=\xi, \mathfrak{N}(t / a) \mathscr{O}_{F}=\xi y d_{F}\right\} / \mathfrak{N}\left(\mathbb{O}_{E, U}^{\times}\right),
$$

which admits a surjection $\pi:(t, u) \mapsto a^{-1} t \mathscr{O}_{E}$ to the set $\boldsymbol{r}_{a^{-1}}\left(\xi y d_{F}\right)$ of ideals $\mathfrak{b} \subset \mathscr{O}_{E}$ in the $U$-class $a^{-1}$, whose norm is $\mathfrak{N}(\mathfrak{b})=\xi y d_{F}$. The fibres of $\pi$ are in bijection with $\mathbb{O}_{E, U}^{\times} / \mathfrak{N}\left(\mathbb{O}_{E, U}^{\times}\right)$, which has cardinality $w_{U}$. We deduce the following description of the Fourier coefficients of $\Theta(\mathcal{W})$ :

Proposition 2.4.1. The series $\Theta(\mathcal{W})$ belongs to $S_{1}\left(K_{1}(\Delta(\mathcal{W})),\left.\varepsilon^{\bar{W}}\right|_{F_{A}^{\times}}\right)$, where $\Delta(\mathcal{W})=\Delta \mathfrak{N}(\mathfrak{f}(\mathcal{W}))$. Its Fourier coefficients are given by

$$
a(\Theta(\mathcal{W}), m)=\sum_{\substack{\mathfrak{b} \subset \mathscr{O}_{E} \\ \mathfrak{N}(\mathfrak{b})=m}} \mathcal{W}(\mathfrak{b})=r_{W}(m)
$$

for $(m, p)=1$ and vanish for $(m, p) \neq 1$.

Corollary 2.4.2. The functional $\Theta$ of Definition 2.3.1 is a measure on $\varphi^{\prime}$ with values in $S_{1}\left(K_{1}(\Delta), \varepsilon\right)$, defined over $\mathbb{Q}_{p}$.

Proof. The distributional property is obvious from the construction or can be seen from the $q$-expansion given above, from which boundedness is also clear. See also [Hida and Tilouine 1993, Theorem 6.2], where a slightly different theta measure is constructed.

Lemma 2.4.3. Assume that $\left(D_{E}, D_{F} p\right)=1$. The theta series admits a functional equation

$$
W_{\Delta(\mathcal{W})} \Theta(\mathcal{W})=(-i)^{[F: \mathbb{Q}]} \mathcal{W}\left(d_{F}^{(p)}\right) \bar{W}\left(\mathfrak{D}_{E}\right) \tau(\bar{W}) \Theta(\bar{W})
$$


where $\mathfrak{D}_{E}$ is the relative different, $d_{F}^{(p)}$ is the prime-to- $p$ factor of the different and $\tau(\bar{W})=\prod_{v \mid p} \tau\left(\bar{W}_{v}\right)$ with

$$
\tau\left(\bar{W}_{v}\right)=\left|\pi_{v}\right|^{-c / 2} \int_{E_{v}^{\times}} \bar{W}_{v}\left(h_{v}\right) \boldsymbol{e}_{v}\left(-\operatorname{Tr}_{E_{v} / F_{v}}\left(h_{v}\right)\right) d h_{v}
$$

if the relative norm of the conductor of $\overline{\mathcal{W}}_{v}$ is $\pi_{v}^{c} \mathcal{O}_{F, v}$.

Proof. Let

$$
\begin{aligned}
& \phi_{W}(g, t, u)=\int_{H(F) \backslash H(A)} \mathcal{W}\left(h^{-1}\right) r(g, h) \phi(t, u) d h, \\
& \phi_{W}^{\prime}(g, t, u)=\varepsilon^{\mathcal{W}}\left(\pi_{\Delta(\mathcal{W})}\right) \int_{H(F) \backslash H(\boldsymbol{A})} \mathcal{W}\left(h^{-1}\right) r\left(g W_{\Delta(\mathcal{W})}, h\right) \phi(t, u) d h
\end{aligned}
$$

for $(t, u) \in E_{A} \times F_{A}^{\times}$. The behaviour in $g$ is through the Weil representation.

Then we have

$$
\begin{aligned}
W_{\Delta(\mathcal{W})} \Theta(\mathcal{W})(g) & =\varepsilon^{\mathscr{W}}(\operatorname{det} g) \sum_{(t, u) \in E \times F^{\times}} \phi_{\mathscr{W}}^{\prime}(g, t, u), \\
\Theta(\bar{W})(g) & =\sum_{(t, u) \in E \times F^{\times}} \phi_{\bar{W}}(g, t, u)
\end{aligned}
$$

so that the lemma follows if we show that for all $(t, u) \in E_{\boldsymbol{A}} \times F_{\boldsymbol{A}}^{\times}$

$$
\varepsilon^{\mathscr{W}}(\operatorname{det} g) \phi_{\mathcal{W}}^{\prime}(g, t, u)=(-i)^{[F: \mathbb{Q}]} \overline{\mathcal{W}}\left(\mathfrak{D}_{E}\right) \tau(\overline{\mathcal{W}}) \varepsilon^{\mathcal{W}}(u) \phi_{\bar{W}}(g, \bar{t}, u)
$$

where $\bar{t}$ is the conjugate of $t$ under the nontrivial automorphism of $E$ over $F$. We write

$$
\tilde{\tau}(\bar{W})=(-i)^{[F: \mathbb{Q}]} \mathcal{W}\left(d_{F}^{(p)}\right)^{\bar{W}}\left(\mathfrak{D}_{E}\right) \tau(\overline{\mathcal{W}})
$$

for short.

We claim that it suffices to prove (2.4.2) for $g=1$. Indeed it is clear that this implies the same result for all $g \in \boldsymbol{S} \boldsymbol{L}_{2}(\boldsymbol{A})$ by acting via the Weil representation on both sides (viewed as functions of $(t, u)$ ). Then it suffices to verify it for the elements of the form $d(y)=\left(\begin{array}{c}1 \\ y\end{array}\right)$ :

$$
\begin{aligned}
\varepsilon(y) \mathcal{W}(y) r(d(y)) \phi_{\mathscr{W}}^{\prime}(1, t, u) & =\tilde{\tau}(\bar{W}) \varepsilon(y) \mathcal{W}(y) r(d(y))\left[\varepsilon^{\mathscr{W}}(u) \phi_{\bar{W}}(1, \bar{t}, u)\right] \\
& =\tilde{\tau}(\bar{W}) \varepsilon^{\mathscr{W}}(y) \varepsilon_{\mathcal{W}} \mathcal{W}\left(y^{-1} u\right) r(d(y)) \phi_{\bar{W}}(1, \bar{t}, u) \\
& =\tilde{\tau}(\bar{W}) \varepsilon(u)^{\mathscr{W}}(u) \phi_{\bar{W}}(d(y), \bar{t}, u) .
\end{aligned}
$$


We now prove (2.4.2) for $g=1$, thus dropping $g$ from the notation. We can write $\phi_{W}^{\prime}(t, u)=\int_{H(F) \backslash H\left(A^{p \Delta}\right)} \mathcal{W}\left(h_{0}^{-1}\right) r(1, h) \phi^{p \Delta}(t, u) d h_{0}$

$$
\cdot \prod_{v \mid p \Delta} \mathcal{W}\left(\pi_{v}^{c_{v}}\right) \int_{H\left(F_{v}\right)} \mathscr{W}\left(h_{v}^{-1}\right) r\left(W_{\pi_{v}^{c_{v}}}, 1\right) \phi\left(h_{v}^{-1} t, v\left(h_{v}\right) u\right) d h_{v}
$$

where $c_{v}$ is the appropriate exponent. We can rewrite this as

$$
\phi_{\mathscr{W}}^{\prime}(t, u)=\phi_{\mathscr{W}}^{\prime \Delta p}(t, u) \prod_{v \mid \Delta p} \phi_{\mathscr{W}, p}^{\prime}(t, u)
$$

with obvious notation. A similar factorisation holds for $\phi_{W}(t, u)$.

For $v \nmid \Delta p$, we have, by the explicit description of $\phi_{v}$ (dropping the subscripts $v$ ), $r(h) \phi(t, u)=\phi\left(h^{-1} t, v(h) u\right)=\phi\left(\pi_{d_{F}} u \bar{h} t, v(h) u\right)$

$$
=\phi\left(\pi_{d_{F}} u h \bar{t}, v(h)^{-1} u^{-1} \pi_{d_{F}}^{-2}\right)=r\left(\left(\pi_{d_{F}} u h\right)^{-1}\right) \phi(\bar{t}, u) .
$$

A change of variable and integration over $H(F) \backslash H\left(A^{\Delta p}\right)$ then gives

$$
\phi_{\mathscr{W}}^{\prime \Delta p}(t, u)=\varepsilon^{\Delta p}\left(u d_{F}\right)^{\mathscr{W}}{ }^{\Delta p}\left(u d_{F}\right) \phi_{\bar{W}}^{\Delta p}(\bar{t}, u) .
$$

For $v \mid \Delta$, we have by (2.5.1) below and the previous argument

$$
\begin{aligned}
\varepsilon(\pi) r\left(W_{\pi}, h\right)(\pi) \phi(t, u)=\varepsilon(u) \kappa(v) \phi\left(h^{-1} t \pi_{\mathfrak{D}}, \pi^{-1} v(h) u\right) & \\
& =\varepsilon(u) \kappa(v) r\left(u^{-1} \pi_{d_{F}^{-1}} h^{-1} \pi_{\mathfrak{D}}\right) \phi(\bar{t}, u)
\end{aligned}
$$

where $\pi_{\mathfrak{D}} \in \mathbb{O}_{E, v}^{\times}$is a generator of the local relative different of $E_{v} / F_{v}$. After change of variable and integration, we obtain

$$
\phi_{\mathscr{W}, v}^{\prime}(t, u)=\kappa(v) \varepsilon_{v}(u)^{\mathscr{W}} \mathcal{W}_{v}\left(u d_{F}\right)^{c} \bar{W}_{v}(\mathfrak{D}) \phi_{\bar{W}, v}(\bar{t}, u) .
$$

For $v \mid p$, we have

$$
\begin{aligned}
\mathscr{W}\left(\pi^{c}\right) & \int_{H\left(F_{v}\right)} \mathscr{W}\left(h^{-1}\right) r\left(h, w_{\pi^{c}}\right) \phi(t, u) d^{\times} h \\
= & |\pi|^{-c / 2} \int_{E^{\times}} \int_{E} \bar{W}\left(\pi^{-c} h\right) \boldsymbol{e}\left(-\pi^{-c} u v(h) \operatorname{Tr}\left(h^{-1} t \bar{\xi}\right)\right) \phi\left(\xi, \pi^{-c} \nu(h) u\right) d \xi d^{\times} h
\end{aligned}
$$

Using the fact that $\phi(\xi, u) d \xi=\phi(\xi, u) d^{\times} \xi$ and a change of variables $\zeta=\pi^{-c} u h \xi \bar{t}$, this equals

$$
\mathscr{W}(u) \tau(\bar{W}) \int_{E^{\times}} \mathscr{W}(\xi \bar{t}) \phi(\xi, v(t \xi) u) d^{\times} \xi
$$

after integration, where the new second argument in $\phi$ gives the condition for the integral in $d \zeta$ to be nonzero. We observe that $\phi(\xi)=\phi\left(\xi^{-1}\right)$ so that with the new 
variable $h^{\prime}=\xi \bar{t}$, and reintroducing $v$ in the notation, this can be rewritten as

$$
\mathscr{W}_{v}(u) \tau\left(\bar{W}_{v}\right) \int_{E_{v}^{\times}} \bar{W}_{v}\left(h_{v}^{\prime}\right)^{-1} \phi\left(h^{\prime-1} \bar{t}, v\left(h_{v}^{\prime}\right) u\right) d^{\times} h_{v}^{\prime}
$$

so that

$$
\phi_{\mathcal{W}, v}^{\prime}(t, u)=\varepsilon_{v}\left(d_{F}\right) \varepsilon_{v}(u) \mathcal{W}_{v}(u) \tau\left(\bar{W}_{v}\right) \phi_{\bar{W}, v}(\bar{t}, u) .
$$

Putting together (2.4.3), (2.4.4) and (2.4.5) and using the formula $\prod_{v \mid \Delta} \kappa(v)=$ $(-i)^{[F: \mathbb{~}]} \mathcal{E}\left(d_{F}\right)$ from [Zhang 2001a, p. 127], ${ }^{20}$ we obtain (2.4.2) as desired.

2.5. Fourier expansion of the theta measure, II. For later use in computing the trace of the convolution of the theta measure with the Eisenstein measure (defined below), we need to consider the expansion of $\Theta\left({ }^{(W}\right)^{(\delta)}(g)=\Theta\left({ }_{W}\right)\left(g W_{\delta}\right)$ for $g=\left(\begin{array}{rr}y & x \\ 1\end{array}\right)$; for such a $g$, we have

$$
\left(\begin{array}{ll}
y & x \\
& 1
\end{array}\right) W_{\delta}=\left(\begin{array}{ll}
1 & x \\
& 1
\end{array}\right)\left(\begin{array}{ll}
y & \\
& \pi_{\delta}
\end{array}\right) w_{\delta}
$$

where $\pi_{\delta}$ is an idèle with components $\pi_{v}$ at $v \mid \delta$ and 1 everywhere else. Here $\pi_{v}$ is a uniformiser chosen to satisfy $\varepsilon\left(\pi_{v}\right)=1$.

The modular form $\Theta(\mathcal{W})^{\delta}$ can be expanded in the same way as in Section 2.4 except that for $v \mid \delta$ we need to replace $\phi_{v}(t, u)=\mathbb{1}_{\mathbb{O}_{E, v}}(t) \mathbb{1}_{d_{F}^{-1, \times}}(u)$ by

$$
\begin{aligned}
W_{\delta} \phi_{v}(t, u) & =\varepsilon_{v}\left(\pi_{v}\right)\left(\begin{array}{ll}
1 & \\
& \pi_{v}
\end{array}\right) \gamma(u) \widehat{\mathbb{1}_{\mathbb{O}_{E, v}}}(t) \mathbb{1}_{d_{F, v}^{-1, \times}}(u) \\
& =\varepsilon_{v}(u) \kappa(v) \mathbb{1}_{\mathfrak{D}_{v}^{-1}}(t) \mathbb{1}_{d_{F}^{-1, \times}}\left(\pi_{\delta}^{-1} u\right) .
\end{aligned}
$$

Here recall that $\mathfrak{D}$ is the relative different of $E / F$ and that $w$ acts as Fourier transform in $t$ with respect to the quadratic form associated with $u \mathfrak{N}$, with the normalising constant $\gamma(u)=\gamma\left(E_{v}, u \mathfrak{N}\right)$ as described in (2.1.1).

The computation of the expansion can then be performed exactly as in Section 2.4. We omit the details but indicate that the relevant substitution is now $a \rightarrow \pi_{\mathfrak{d}} a y$, where $\mathfrak{d}$ is an ideal of $\widehat{O}_{E}$ of norm $\delta$ and $\pi_{\mathfrak{d}} \in \widehat{\mathrm{O}_{E}}$ is a generator with components equal to 1 away from $\mathfrak{d}$.

Proposition 2.5.1. The Whittaker-Fourier coefficients of the series $\Theta(\mathcal{W})^{(\delta)}$ are given by

$$
\tilde{a}\left(\Theta(\mathcal{W})^{(\delta)}, y\right)=\varepsilon^{\mathscr{W}}(y)|y|^{1 / 2} \kappa(\delta) \mathcal{W}(\mathfrak{d}) \varepsilon_{\delta}(y) r_{W}\left(y d_{F}\right),
$$

where $\kappa(\delta)=\prod_{v \mid \delta} \kappa(v)$.

${ }^{20}$ Recall that our $\kappa(v)$ are the inverses of the $\kappa(v)$ of [loc. cit.]. 


\section{Eisenstein measure}

In this section, we construct a measure (see Section 2.3) valued in Eisenstein series of weight 1 and compute its Fourier expansion.

3.1. Eisenstein series. Let $k$ be a positive integer, $M$ be an ideal of $\mathscr{O}_{F}$, and $\varphi: F_{A}^{\times} / F^{\times} \rightarrow \mathbb{C}^{\times}$be a finite-order character of conductor dividing $M$ satisfying $\varphi_{v}(-1)=(-1)^{k}$ for $v \mid \infty$. Let

$$
L^{M}(s, \varphi)=\sum_{(m, M)=1} \varphi(m) N(m)^{-s},
$$

where the sum runs over all nonzero ideals of $O_{F}$.

Let $B \subset \boldsymbol{G L}_{2}$ be the Borel subgroup of upper-triangular matrices; recall the notation from Section 1.1 and the Iwasawa decomposition (1.1.1); the decomposition is not unique, but the ideal of $\hat{O}_{F}$ generated by the lower-left entry of the $K_{0}(1)$ component is well-defined.

For $s \in \mathbb{C}$, define a function $H_{k, s}(g, \varphi)$ on $\boldsymbol{G} \boldsymbol{L}_{2}\left(\boldsymbol{A}_{F}\right)$ by

$$
H_{k, s}(g=q u r(\theta) ; \varphi)=\left\{\begin{array}{cl}
\left|y_{1} / y_{2}\right|^{s} \varphi\left(y_{1} a\right) \boldsymbol{e}_{\infty}(k \theta) & \text { if } u=\left(\begin{array}{ll}
a & b \\
c & d
\end{array}\right) \in K_{0}(M), \\
0 & \text { if } u \in K_{0}(1) \backslash K_{0}\left(M_{0}\right),
\end{array}\right.
$$

where $g=q u r(\theta)$ with $q=\left(\begin{array}{cc}y_{1} & x \\ & y_{2}\end{array}\right) \in B\left(\boldsymbol{A}_{F}\right), u \in K_{0}(1)$ and $r(\theta) \in K_{\infty}$.

We define two Eisenstein series

$$
\begin{aligned}
& E_{k}^{M}(g, s ; \varphi)=L^{M}(2 s, \varphi) \sum_{\gamma \in B(F) \backslash G L_{2}(F)} H_{k, s}(\gamma g ; \varphi), \\
& \widetilde{E}_{k}^{M}(g, s ; \varphi)=W_{M} E_{k}^{M}(g, s ; \varphi)=\varphi^{-1}\left(\operatorname{det} g \pi_{M}\right) E_{k}^{M}\left(g W_{M}, s ; \varphi\right),
\end{aligned}
$$

which are absolutely convergent for $\operatorname{Re} s>1$ and continue analytically for all $s$ to (nonholomorphic) automorphic forms of level $M$, parallel weight $k$ and character $\varphi$ (for $E$ ) and $\varphi^{-1}$ (for $\widetilde{E}$ ). Here $W_{M}$ is as in (1.4.3). The superscript $M$ will be omitted from the notation when its value is clear from context.

3.2. Fourier expansion of the Eisenstein measure. We specialise to the case where $k$ is odd, $M=\Delta P$ with $(\Delta, P)=1$ and $\varphi=\varepsilon \phi$ with $\varepsilon=\varepsilon_{E / F}$ and $\phi$ a character of conductor dividing $P$, trivial at infinity (in particular, we have $\varphi_{v}(-1)=\varepsilon_{v}(-1) \phi_{v}(-1)=-1$ as required). We assume that $\Delta$ is squarefree. For $\delta \mid \Delta$, we compute ${ }^{21}$ the Whittaker coefficients (see Section 1.2; we suppress $\varphi, M$ and $k$ from the notation) of $\widetilde{E}^{(\delta)}$ :

$$
c_{s}^{\delta}(\alpha, y)=D_{F}^{-1 / 2} \int_{A_{F} / F} \widetilde{E}\left(\left(\begin{array}{rr}
y & x \\
& 1
\end{array}\right) W_{\delta}, s\right) \boldsymbol{e}(-\alpha x) d x
$$

${ }^{21}$ See [Zhang 2001a, §3.5, §6.2]. 
for $\alpha \in F$ and $\delta$ dividing $\Delta$; since $c_{s}(\alpha, y)=c_{s}(1, \alpha y)$ for $\alpha \neq 0$, we can restrict to $\alpha=0$ or 1 . The choice of uniformisers $\pi_{v}$ at $v \mid \delta$ implicit in the above formula is made so that $\varepsilon\left(\pi_{v}\right)=1$ to save some notation.

Proposition 3.2.1. In the case just described, the Whittaker coefficients $c_{s}^{\delta}(\alpha, y)$ of the Eisenstein series $\widetilde{E}_{k}^{(\delta)}(g, s ; \varphi)$ are given by

$$
\begin{aligned}
& c_{s}^{\delta}(0, y)=\left\{\begin{array}{cc}
\frac{1}{D_{F}^{1 / 2} N(\Delta P)^{s}} \varepsilon \phi(y)|y|^{1-s} V_{k, s}(0)^{[F: \mathbb{Q}]} L^{(P)}(2 s-1, \varepsilon \phi) & \text { if } \delta=1, \\
0 & \text { if } \delta \neq 1,
\end{array}\right. \\
& c_{s}^{\delta}(1, y)=\left\{\begin{array}{cl}
\frac{N(\delta)^{s-1 / 2}}{D_{F}^{1 / 2} N(\Delta P)^{s}} \cdot \phi(y)|y|^{1-s} \kappa(\delta) \phi(\delta) \varepsilon_{\delta}(y) & \text { otherwise, } \\
\cdot \phi_{\delta}\left(y^{\infty} d_{F}\right)\left|y \pi_{\delta} d_{F}\right|_{\delta}^{2 s-1} \sigma_{k, s, \varepsilon \phi}(y) & \text { if } y d_{F} \text { is integral } \\
0 & \text { other }
\end{array}\right.
\end{aligned}
$$

where $\kappa(\delta)=\prod_{v \mid \delta} \kappa(v)$ with $\kappa(v)$ as in (2.1.1) and

$$
\sigma_{k, s, \varphi}(y)=\prod_{v \nmid \Delta M \infty} \sum_{n=0}^{v\left(y d_{F}\right)} \varphi_{v}\left(\pi_{v}\right)^{n}\left|\pi_{v}\right|^{n(2 s-1)} \prod_{v \mid \infty} V_{k, s}\left(y_{v}\right)
$$

with

$$
V_{k, s}(y)=\int_{\mathbb{R}} \frac{e^{-2 \pi i y x}}{\left(x^{2}+1\right)^{s-k / 2}(x+i)^{k}} d x .
$$

Proof. We use the Bruhat decomposition

$$
\boldsymbol{G L}_{2}(F)=B(F) \bigsqcup B(F) w N(F)
$$

with $w=\left({ }_{1}{ }^{-1}\right)$ and the unipotent subgroup $N(F) \cong F$ via $N(F) \ni\left(\begin{array}{rr}1 & x \\ 1\end{array}\right) \leftrightarrow x \in F$ to get

$$
\begin{aligned}
\varepsilon \phi(y) \phi\left(\pi_{M / \delta}\right) c_{s}^{\delta}(\alpha, y)= & L(2 s, \varphi) D_{F}^{-1 / 2} \int_{\boldsymbol{A}_{F} / F} H_{s}\left(\left(\begin{array}{rr}
y & x \\
& 1
\end{array}\right) W_{M / \delta}\right) \boldsymbol{e}(-\alpha x) d x \\
& +L(2 s, \varphi) D_{F}^{-1 / 2} \int_{\boldsymbol{A}_{F}} H_{s}\left(w\left(\begin{array}{rr}
y & x \\
& 1
\end{array}\right) W_{M / \delta}\right) \boldsymbol{e}(-\alpha x) d x .
\end{aligned}
$$

At any place $v \mid M / \delta$, we have the decomposition

$$
\left(\begin{array}{cc}
y_{v} & x_{v} \\
& 1
\end{array}\right) W_{M / \delta, v}=\left(\begin{array}{cc}
y_{v} & \pi_{v} x_{v} \\
& \pi_{v}
\end{array}\right)\left(\begin{array}{cc} 
& 1 \\
-1 &
\end{array}\right)
$$

so that the first summand is always zero.

For the second integral, we use the identity

$$
w\left(\begin{array}{ll}
y & x \\
& 1
\end{array}\right)=\left(\begin{array}{ll}
1 & \\
& y
\end{array}\right)\left(\begin{array}{cc}
-1 \\
1 & x y^{-1}
\end{array}\right)
$$


and the substitution $x \rightarrow x y$ to get

$$
\int_{A_{F}} H_{s}\left(w\left(\begin{array}{ll}
y & x \\
& 1
\end{array}\right) W_{M / \delta}\right) \boldsymbol{e}(-\alpha x) d x=|y|^{1-s} \prod_{v} V_{s}^{M / \delta}\left(\alpha_{v} y_{v}\right),
$$

where for $y \in F_{v}$

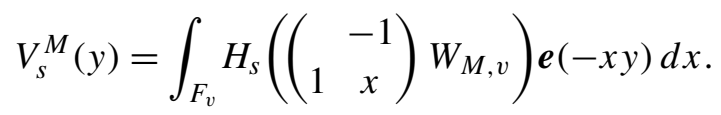

Archimedean places. See [Zhang 2001a, Proposition 3.5.2].

Nonarchimedean places $v \nmid M / \delta$. If $v$ is a finite place, we have $\left(\begin{array}{cc}-1 \\ 1 & x\end{array}\right) \in \boldsymbol{G L}_{2}\left(\boldsymbol{O}_{F, v}\right)$ if $x \in O_{F, v}$, and otherwise we have the decomposition

$$
\left(\begin{array}{cc}
-1 \\
1 & x
\end{array}\right)=\left(\begin{array}{cc}
x^{-1} & -1 \\
& x
\end{array}\right)\left(\begin{array}{cc}
1 & \\
x^{-1} & 1
\end{array}\right)
$$

Therefore,

$$
H_{s, v}\left(\begin{array}{c}
-1 \\
1 \quad x
\end{array}\right)=\left\{\begin{array}{cl}
\bar{\varphi}_{v}(x)|x|^{-2 s} & \text { if } v(x) \leq-1 \\
1 & \text { if } v \nmid M \text { and } v(x) \geq 0 \\
0 & \text { if } v \mid \delta \text { and } v(x) \geq 0 .
\end{array}\right.
$$

The case $v \nmid M$. We deduce that

$$
\begin{aligned}
V_{s}^{M / \delta}(y) & =\int_{\mathscr{O}_{F, v}} \boldsymbol{e}(-x y) d x+\sum_{n \geq 1} \int_{\mathscr{O}_{F, v}^{\times}} \bar{\varphi}_{v}\left(x \pi_{v}^{-n}\right)\left|x \pi_{v}^{-n}\right|^{-2 s} \boldsymbol{e}\left(-x y \pi_{v}^{-n}\right) d\left(\pi_{v}^{-n} x\right) \\
& =\mathbb{1}\left[y \in d_{F}^{-1}\right]+\sum_{n \geq 1} \varphi_{v}\left(\pi_{v}\right)^{n}\left|\pi_{v}\right|^{n(2 s-1)} \int_{\mathscr{O}_{F, v}^{\times}} \boldsymbol{e}\left(-x y \pi_{v}^{-n}\right) d x .
\end{aligned}
$$

The integral evaluates to $1-\left|\pi_{v}\right|$ if $v\left(y d_{F}\right) \geq n$, to $-\left|\pi_{v}\right|$ if $v\left(y d_{F}\right)=n-1$ and to 0 otherwise. Therefore, we have $V_{s}^{M}(y)=0$ unless $v\left(y d_{F}\right) \geq 0$, in which case if $y \neq 0$

$$
\begin{aligned}
V_{s}^{M / \delta}(y) & =1+\left(1-\left|\pi_{v}\right|\right) \sum_{n=1}^{v\left(y d_{F}\right)}\left(\varphi_{v}\left(\pi_{v}\right)\left|\pi_{v}\right|^{2 s-1}\right)^{n}-\left|\pi_{v}\right|\left(\varphi_{v}\left(\pi_{v}\right)\left|\pi_{v}\right|^{2 s-1}\right)^{v\left(y d_{f}\right)+1} \\
& =\left(1-\varphi_{v}\left(\pi_{v}\right)\left|\pi_{v}\right|^{2 s}\right) \sum_{n=0}^{v\left(y d_{F}\right)} \varphi_{v}\left(\pi_{v}\right)^{n}\left|\pi_{v}\right|^{n(2 s-1)} \\
& =L_{v}(2 s, \varphi)^{-1} \sum_{n=0}^{v\left(y d_{F}\right)} \varphi_{v}\left(\pi_{v}\right)^{n}\left|\pi_{v}\right|^{n(2 s-1)}
\end{aligned}
$$


whereas for $y=0$ we have

$$
\begin{aligned}
V_{s}^{M / \delta}(0) & =1+\left(1-\left|\pi_{v}\right|\right) \sum_{n=1}^{\infty}\left(\varphi_{v}\left(\pi_{v}\right)\left|\pi_{v}\right|^{2 s-1}\right)^{n} \\
& =1+\left(1-\left|\pi_{v}\right|\right)\left(1-\varphi_{v}\left(\pi_{v}\right)\left|\pi_{v}\right|^{2 s-1}\right)^{-1}\left(1-\varphi_{v}\left(\pi_{v}\right)\left|\pi_{v}\right|^{2 s}\right) \\
& =L_{v}(2 s, \varphi)^{-1} L_{v}(2 s-1, \varphi) .
\end{aligned}
$$

The case $v \mid \delta$. Again by (3.2.2), we find

$$
V_{s}^{M / \delta}(y)=\sum_{n \geq 1} \int_{\mathscr{O}_{F, v}^{\times}} \bar{\varphi}_{v}\left(x \pi_{v}^{-n}\right)\left|x \pi_{v}^{-n}\right|^{-(2 s-1)} \boldsymbol{e}\left(-x y \pi_{v}^{-n}\right) d x .
$$

All the integrals vanish except the one with $n=v\left(y d_{F}\right)+1$, which gives

$$
\varepsilon_{v}\left(y \pi_{v}^{n}\right) \phi_{v}\left(y \pi_{d_{F}, v} \pi_{v}\right)\left|y \pi_{d_{F}, v} \pi_{v}\right|^{2 s-1}\left|\pi_{v}\right|^{1 / 2} \kappa(v) ;
$$

therefore, we have ${ }^{22}$

$$
V_{s}^{M / \delta}(y)=\varepsilon_{v}(y) \phi_{v}\left(y \pi_{d_{F}, v} \pi_{v}\right)\left|y \pi_{d_{F}, v} \pi_{v}\right|^{2 s-1}\left|\pi_{v}\right|^{1 / 2} \kappa(v)
$$

if $y \neq 0$ and $v\left(y d_{F}\right) \geq 0$ and $V_{s}(y)=0$ otherwise. In particular, we see that if $\delta \neq 1$ then $V_{s}(0)=c_{S}(0, y)=0$.

Places $v \mid M / \delta$. For

$$
w\left(\begin{array}{ll}
1 & x \\
& 1
\end{array}\right)\left(\begin{array}{cc} 
& 1 \\
-\pi_{v}^{v(M)} &
\end{array}\right)=\left(\begin{array}{cc}
\pi_{v}^{v(M)} & \\
-x \pi_{v}^{v(M)} & 1
\end{array}\right)
$$

we have the decompositions

$$
\begin{aligned}
\left(\begin{array}{cc}
\pi_{v}^{v(M)} & \\
-x \pi_{v}^{v(M)} & 1
\end{array}\right) & =\left(\begin{array}{ll}
-\pi_{v}^{v(M)} & \\
& 1
\end{array}\right)\left(\begin{array}{cc}
-1 & \\
-x \pi_{v}^{v(M)} & 1
\end{array}\right) \\
& =\left(\begin{array}{cc}
x^{-1} & -\pi_{v}^{v(M)} \\
x \pi_{v}^{v(M)}
\end{array}\right)\left(\begin{array}{cc}
1 \\
-1 & x^{-1} \pi_{v}^{-v(M)}
\end{array}\right) ;
\end{aligned}
$$

for $v(x) \geq 0$, we use the first one to find

$$
H_{s}\left(\begin{array}{ll}
-\pi_{v}^{v(M)} & \\
x \pi_{v}^{v(M)} & -1
\end{array}\right)=\varphi_{v}\left(\pi_{v}\right)^{v(M)}\left|\pi_{v}^{v(M)}\right|^{s}
$$

for $v(x)<0$, the second decomposition shows that the integrand vanishes. We conclude that

$$
V_{s}^{M / \delta}(y)=\left\{\begin{array}{cl}
\varphi_{v}\left(\pi_{v}\right)^{v(M)}\left|\pi_{v}^{v(M)}\right|^{s} & \text { if } v\left(y d_{F}\right) \geq 0, \\
0 & \text { otherwise. }
\end{array}\right.
$$

The final formula follows from these computations.

\footnotetext{
${ }^{22}$ Recall that we always choose $\pi_{v}$ so that $\varepsilon_{v}\left(\pi_{v}\right)=1$.
} 
We specialise to the case $s=\frac{1}{2}$ and consider the rescaled holomorphic Eisenstein series: ${ }^{23}$

$$
\begin{aligned}
& \boldsymbol{E}_{k, \varepsilon \phi}^{\Delta P}(g)=\frac{D_{F}^{1 / 2} N(\Delta P)^{1 / 2}}{(-2 \pi i)^{[F: \mathbb{Q}]}} E_{k}^{\Delta P}\left(g, \frac{1}{2} ; \varepsilon \phi\right), \\
& \widetilde{\boldsymbol{E}}_{k, \varepsilon \phi}^{\Delta P}(g)=\frac{D_{F}^{1 / 2} N(\Delta P)^{1 / 2}}{(-2 \pi i)^{[F: \mathbb{Q}]}} \widetilde{E}_{k}^{\Delta P}\left(g, \frac{1}{2} ; \varepsilon \phi\right) .
\end{aligned}
$$

We further specialise to the case $k=1$.

Corollary 3.2.2. The Eisenstein series $\widetilde{\boldsymbol{E}}_{1, \varepsilon \phi}^{\Delta P}$ belongs to $M_{1}\left(K_{1}(\Delta P), \varepsilon \phi^{-1}\right)$. The Whittaker-Fourier coefficients of $\widetilde{\boldsymbol{E}}_{\varepsilon \phi}^{(\delta)}=\widetilde{\widetilde{E}}_{1, \varepsilon \phi}^{\Delta P,(\delta)}$ for $\delta \mid \Delta$ are given by

$$
\tilde{a}^{0}\left(\widetilde{\boldsymbol{E}}_{\varepsilon \phi}^{(\delta)}, y\right)=\varepsilon \phi(y)|y|^{1 / 2} \frac{L^{(p)}(0, \varepsilon \phi)}{2^{g}}
$$

if $\delta=1$ and $\tilde{a}^{0}\left(\widetilde{\boldsymbol{E}}_{\varepsilon \phi}^{(\delta)}, y\right)=0$ otherwise, and

$$
c^{\delta}(y)=\tilde{a}\left(\widetilde{\boldsymbol{E}}_{\varepsilon \phi}^{(\delta)}, y\right)=\varepsilon \phi(y)|y|^{1 / 2} \kappa(\delta) \phi(\delta) \varepsilon_{\delta} \phi_{\delta}\left(y^{\infty} d_{F}\right) \sigma_{\varepsilon \phi}\left(y^{\infty} d_{F}\right),
$$

where for any integral ideal $m$ of $O_{F}\left[\Delta^{-1} P^{-1}\right]$

$$
\sigma_{\varepsilon \phi}(m)=\sum_{d \mid m} \varepsilon \phi(d)
$$

the sum likewise running over integral ideals of $\mathrm{O}_{F}\left[\Delta^{-1} P^{-1}\right]$.

(If $m$ is an integral ideal of $\mathscr{O}_{F}$ prime to $P$, then $\sigma_{\varepsilon \mathbb{1}}(m)=r(m)$.)

Proof. This follows from Proposition 3.2.1 together with the evaluation

$$
V_{1,1 / 2}(t)=\left\{\begin{array}{cc}
0 & \text { if } t<0 \\
-\pi i & \text { if } t=0 \\
-2 \pi i e^{-2 \pi t} & \text { if } t>0
\end{array}\right.
$$

which can be found in [Gross and Zagier 1986, Proposition IV.3.3 (a) and (d)] (for the case $t=0$, this is deduced from (a) of [loc. cit.] using $\lim _{s \rightarrow 0} \Gamma(2 s) / \Gamma(s)=\frac{1}{2}$ ).

Definition 3.2.3. Let $F_{\infty}^{\prime}$ be the maximal abelian extension of $F$ unramified outside $p$, and let $\mathscr{G}_{F}^{\prime}=\operatorname{Gal}\left(F_{\infty}^{\prime} / F\right)$. We define the Eisenstein (pseudo)measure ${ }^{24} \widetilde{\boldsymbol{E}}_{\varepsilon}$ on $\mathscr{G}_{F}^{\prime}$ by

$$
\widetilde{\boldsymbol{E}}_{\varepsilon}(\phi)=\widetilde{\boldsymbol{E}}_{\varepsilon \phi}^{\Delta P}=\frac{D_{F}^{1 / 2} N(\Delta P)^{1 / 2}}{(-2 \pi i)^{g}} \widetilde{E}_{\varepsilon \phi}^{\Delta P}
$$

${ }^{23}$ Notice that these series do not depend on the ideal $P$ but only on its support.

${ }^{24} \mathrm{We}$ do not need to assume that $\Delta$ is squarefree when making the definition. See after the definition for the meaning of the term pseudomeasure. 
for any character $\phi$ of $G_{F}^{\prime}$ of conductor dividing $P$ (it does not depend on the choice of $P$ once we require $P$ to satisfy $v|P \leftrightarrow v| p$ ). We denote with the same name the distribution induced on the group $\mathscr{G}^{\prime}$ of Section 2.3 by

$$
\widetilde{\boldsymbol{E}}_{\varepsilon}(\mathcal{W})=\widetilde{\boldsymbol{E}}_{\varepsilon}\left(\left.\mathcal{W}\right|_{F_{A}^{\times}}\right) .
$$

It has values in $\boldsymbol{M}_{1}\left(K_{1}(N \Delta), \varepsilon\right)$ and is defined over $\mathbb{Q}_{p}$.

To prove the soundness of the definition, it is easy to see that the nonzero Fourier coefficients interpolate to a measure on $\varphi_{F}^{\prime}$, that is, an element of $\mathbb{Z}_{p} \llbracket \varphi_{F}^{\prime} \rrbracket$. The $L$ values giving the constant term interpolate to the Deligne-Ribet $p$-adic $L$-function [1980]; it is a pseudomeasure in the sense of Serre [1978], that is, an element of the total quotient ring of $\mathbb{Z}_{p} \llbracket G_{F}^{\prime} \rrbracket$ with denominators of a particularly simple form.

\section{The $p$-adic $L$-function}

4.1. Rankin-Selberg convolution. Let $f$ and $g$ be modular forms of common level $M$, weights $k_{f}$ and $k_{g}$ and characters $\psi_{f}$ and $\psi_{g}$, respectively. We define a normalised Dirichlet series

$$
D^{M}(f, g, s)=L^{M}\left(2 s-1, \psi_{f} \psi_{g}\right) \sum_{m} a(f, m) a(g, m) N m^{-s},
$$

where the imprimitive $L$-function $L^{M}(s, \varphi)$ of a Hecke character $\varphi$ of conductor dividing $M$ is as in (3.1.1).

When $f$ and $g$ are primitive forms of level $N_{f}$ and $N_{g}$ (that is, normalised new eigenforms at those levels), for a prime $\wp \nmid N_{f}$, denote by $\gamma_{\wp}^{(1)}(f)$ and $\gamma_{\wp}^{(2)}(f)$ the two roots of the $\wp$-th Hecke polynomial of $f$

$$
P_{\wp, f}(X)=X^{2}-a(f, \wp) X+\psi_{f}(\wp) N \wp^{k_{f}-1}
$$

and by $\gamma_{\wp}^{(1)}(g)$ and $\gamma_{\wp}^{(2)}(g)$ the analogous quantities for $g$. Then the degree-4 Rankin-Selberg $L$-function $L(f \times g, s)$ with unramified Euler factors at $\wp$ given by

$$
\prod_{i, j=1}^{2}\left(1-\gamma_{\wp}^{(i)}(f) \gamma_{\wp}^{(j)}(g) N_{\wp} \wp^{-s}\right)^{-1}
$$

equals the above Dirichlet series

$$
L(f \times g, s)=D^{N_{f} N_{g}}(f, g, s)
$$

if $N_{f}$ and $N_{g}$ are coprime. 
Suppose now for simplicity that $k_{f}=2, k_{g}=1$ and $f$ is a cusp form (not necessarily primitive). The Rankin-Selberg convolution method ${ }^{25}$ gives

$$
\left\langle f^{\rho}, g E_{1}^{M}\left(s ; \psi_{f} \psi_{g}\right)\right\rangle_{M}=D_{F}^{s+1}\left[\frac{\Gamma\left(s+\frac{1}{2}\right)}{(4 \pi)^{s+1 / 2}}\right]^{[F: \mathbb{Q}]} D^{M}\left(f \times g, s+\frac{1}{2}\right),
$$

where $\langle\cdot, \cdot\rangle_{M}$ is the Petersson inner product (1.1.2).

4.2. Convoluted measure and the p-adic L-function in the ordinary case. Consider the convolution pseudomeasure $\Theta * \widetilde{\boldsymbol{E}}_{\varepsilon, N}$ on $\boldsymbol{G}^{\prime}$ defined by $\Theta * \widetilde{\boldsymbol{E}}_{\varepsilon, N}\left({ }^{(W}\right)=$ $\Theta(\mathcal{W}) \widetilde{\boldsymbol{E}}_{\varepsilon, N}(\bar{W})$ for any character $\mathscr{W}: \mathscr{G}^{\prime} \rightarrow \mathbb{Z}_{p}^{\times}$, where $\widetilde{\boldsymbol{E}}_{\varepsilon, N}=[N] \widetilde{\boldsymbol{E}}_{\varepsilon}$. We deduce from it the (pseudo)measure

$$
\Phi(\mathcal{W})=\operatorname{Tr}_{\Delta}\left[\Theta * \widetilde{\boldsymbol{E}}_{\varepsilon, N}(\bar{W})\right]=\operatorname{Tr}_{\Delta}\left[\Theta(\mathcal{W}) \cdot[N] \widetilde{\boldsymbol{E}}_{\varepsilon}(\bar{W})\right]
$$

on $\varphi^{\prime}$, which is a kind of $p$-adic kernel of the Rankin-Selberg $L$-function as will be made precise below. It is valued in $\boldsymbol{M}_{2}\left(K_{0}(N), \mathbb{C}_{p}\right)$. Notice that, while $\Phi(\mathcal{W})$, like $\widetilde{\boldsymbol{E}}_{\varepsilon, N}$, is not a measure, we can see that, for any $\wp \mid p$,

$$
U_{\wp} \Phi(\mathcal{W})
$$

is. Indeed its Fourier coefficients are the Fourier coefficients of $\Phi(\mathcal{W})$ at ideals $m$ divisible by $\wp$ and hence sums of coefficients of the theta and Eisenstein series at pairs of ideals $\left(m_{1}=n m, m_{2}=(1-n) m\right)$ for some $n \in F$; since the coefficients of the theta series are zero at ideals $m_{1}$ divisible by $\wp$, only those pairs $\left(m_{1}, m_{2}\right)$ with $m_{1}$ and $m_{2}$ both prime to $\wp$ contribute. In particular, the constant term of the Eisenstein series does not contribute to the Fourier expansion of $U_{\wp} \Phi$, which therefore belongs to $\mathbb{Z}_{p} \llbracket \mathscr{G}^{\prime} \rrbracket \otimes S_{2}\left(K_{0}(N), \mathbb{C}_{p}\right)$.

Thanks to this discussion and the identity $l_{f_{\alpha}}=\alpha_{\wp}^{-1} l_{f_{\alpha}} \circ U_{\wp}$, the following definition makes sense:

Definition 4.2.1. The $p$-adic Rankin-Selberg $L$-function is an element of $O_{L} \llbracket \varphi \mathscr{\llbracket} \rrbracket \otimes L$ that is defined by

$$
L_{p}\left(f_{E}, \mathcal{W}\right)=D_{F}^{-2} H_{p}(f) l_{f_{\alpha}}(\Phi(\mathcal{W}))
$$

for any character $\mathcal{W}: \mathscr{G} \rightarrow \mathrm{O}_{L}^{\times}$, where

$$
H_{p}(f)=\prod_{\wp \mid p}\left(1-\frac{1}{\alpha_{\wp}(f)^{2}}\right)\left(1-\frac{N_{\wp}}{\alpha_{\wp}(f)^{2}}\right) .
$$

${ }^{25}$ See [Shimura 1978] or [Jacquet 1972, Chapter V] for general treatments; our setting and normalisations are the same as in [Zhang 2001a, Lemma 6.1.3] (where $g$ is a specific form, but the same calculation works in general to prove (4.1.1)). 
Functional equation. The $p$-adic $L$-function admits a functional equation; we prove it in the case of anticyclotomic characters, which is the only one we shall need.

Proposition 4.2.2. Suppose $\mathcal{W}$ is an anticyclotomic character of $\mathscr{G}$, i.e., $\left.\mathscr{W}\right|_{F_{A^{\times}}}=1$. Then there are functional equations for the p-adic L-function

$$
L_{p}\left(f_{E}\right)(\mathcal{W})=(-1)^{g} \varepsilon(N) L_{p}(\mathcal{W})
$$

and for the analytic kernel

$$
\Phi(\mathcal{W})=(-1)^{g} \varepsilon(N) \Phi(\mathcal{W}) .
$$

In particular, if $\varepsilon(N)=(-1)^{g-1}$, we have

$$
\Phi(\mathscr{W})=L_{p}\left(f_{E}\right)(\mathscr{W})=0 .
$$

Proof. The functional equation for $L_{p}$ is implied by the functional equation for $\Phi$. We prove the latter by comparing the coefficients on both sides. From (4.5.1) below, ${ }^{26}$ the coefficients of $\Phi(\mathcal{W})$ are given by

$$
b(m)=\sum_{\delta \mid \Delta} \sum_{\substack{n \in F \\ 0<n<1}} \varepsilon_{\delta}((n-1) n) r_{W^{-}}((1-n) m \delta) \sigma_{\varepsilon \mathbb{1}}(n m / N) .
$$

(We use the notation $\mathbb{1}$ for the character of ideals defined by $\mathbb{1}(m)=1$ if $(m, p)=1$ and $\mathbb{1}(m)=0$ otherwise.) We rewrite this as $b(m)=\sum_{\delta, n} b_{\delta, n}(m)$ with, using $\varepsilon_{\delta}(x)=\varepsilon^{\delta}(x)$ for $x \in F^{\times}$and writing in columns to highlight the factors,

$$
\begin{aligned}
& b_{\delta, n}(m)=\varepsilon_{\delta}(-1) \\
& =(-1)^{g} \varepsilon_{\Delta / \delta}(-1) \\
& \cdot \varepsilon_{\Delta / \delta}((1-n) m) \varepsilon_{\Delta / \delta}(n m) \quad \cdot \varepsilon_{\Delta / \delta}((1-n) m) \varepsilon_{\Delta / \delta}(n m) \\
& \cdot \varepsilon^{\Delta}((1-n) m) r_{W}((1-n) m \delta) \cdot r_{W}((1-n) m \Delta / \delta) \\
& \cdot \varepsilon(N) \\
& \cdot \varepsilon(N) \\
& \cdot \varepsilon^{\Delta}(n m / N) \sigma_{\varepsilon \mathbb{1}}(n m / N) \\
& \cdot \sigma_{\varepsilon \mathbb{1}}(n m / N)=(-1)^{g} \varepsilon(N) b_{\Delta / \delta, n} .
\end{aligned}
$$

Here we have used the following facts. In the first line, $\varepsilon_{\Delta}(-1)=\varepsilon_{\infty}(-1)=$ $(-1)^{g}$. In the third line, we have that $r_{W}(m)=1$ if $m$ is divisible only by ramified primes in $E$ since in that case $m=\mathfrak{m}^{2}$ is a square and $\mathscr{W}(\mathfrak{m})^{2}=\mathscr{W}(m)=1-$ this implies $\mathscr{W}(\mathfrak{m})= \pm 1$ and hence $\mathscr{W}(\mathfrak{m})=1$ since $\mathscr{W}$, which is a character of $\mathscr{G} \cong \mathbb{Z}_{p}^{1+g+\delta}$, has values in $1+p \mathbb{Z}_{p}$. Finally, in the third and fifth lines, one can observe that, if $q=\sigma_{\varepsilon \mathbb{1}}$ or $q=r$, then $\varepsilon^{\Delta}(m) q(m)=q(m)$; indeed this is trivial if $\varepsilon^{\Delta}(m)=1$ while both sides are zero if $\varepsilon^{\Delta}(m)=-1$.

${ }^{26}$ Which does not use the present result. The formula (4.5.1) is stated in the case when the anticyclotomic part $\mathcal{W}^{-}=\mathbb{1}$, but the very same calculation gives the result in general. 
4.3. Interpolation property. We manipulate the definition to show that the $p$-adic $L$-function $L_{p}\left(f_{E}\right)(\mathcal{W})$ of Definition 4.2.1 interpolates the special values of the complex Rankin-Selberg $L$-function $L\left(f_{E}, \mathscr{W}, s\right)=L(f \times \Theta(\mathscr{W}), s)$ defined in the introduction.

We will need a few technical lemmas.

Lemma 4.3.1. Let $P$ be an ideal of $O_{F}$ such that $v \mid P$ if and only if $v \mid p$. We have

$$
\left\langle W_{N P} f_{\alpha}^{\rho}, f_{\alpha}\right\rangle_{N P}=\alpha_{P}(f)(-1)^{g} \tau(f) H_{p}(f)\langle f, f\rangle_{N}
$$

with $H_{p}(f)$ as in (4.2.2) and

$$
\alpha_{P}(f)=\prod_{\wp \mid p} \alpha_{\wp}(f)^{v_{\wp}(P)} .
$$

Proof. When $P=P_{0}:=\prod_{\wp \mid p} \wp$, this is the direct generalisation of [Perrin-Riou 1988 , Lemme 27], and it is proved in the same way. In general, we can write $P=P_{0} P_{1}$, and then

$$
W_{N P} f_{\alpha}^{\rho}=N\left(P_{1}\right)\left[P_{1}\right] W_{N P_{0}} f_{\alpha}^{\rho} .
$$

Observing that $[\wp]$ is the adjoint of $U_{\wp}$ for the Petersson inner product and that $\boldsymbol{N}\left(P_{1}\right)=\left[K_{0}(N P): K_{0}\left(N P_{0}\right)\right]$, we deduce

$$
\begin{aligned}
\left\langle W_{N P} f_{\alpha}^{\rho}\right\rangle_{N P}=\left[K_{0}(N P): K_{0}\left(N P_{0}\right)\right]\left\langle W_{N P_{0}} f_{\alpha}^{\rho}, U\left(P_{1}\right)\right. & \left.f_{\alpha}\right\rangle_{N P} \\
& =\alpha_{P_{1}}\left\langle W_{N P_{0}} f_{\alpha}^{\rho}, f_{\alpha}\right\rangle_{N P_{0}} .
\end{aligned}
$$

The lemma then follows from this and the special case $P=P_{0}$.

For the next lemma, let $M$ and $N$ be coprime; then we define the space of weakly $N$-old forms of level $N M$ to be the subspace of $M_{k}\left(K_{1}(M N)\right)$ spanned by forms $f=[d] f^{\prime}$ for some $d \mid N$ and some modular form $f^{\prime}$ of level $N^{\prime} M$ with $N^{\prime} \mid d^{-1} N$. (This is often simply called the space of $N$-old forms, but we have reserved that name for the span of forms $[d] f^{\prime}$ as above with $d \neq 1$.)

Lemma 4.3.2. For a character $\varphi$ of conductor dividing $M$ and an ideal $N$ prime to $M$, let $E_{\varphi}^{M}=E_{1}^{M}\left(g, \frac{1}{2} ; \varphi\right)$ and $\widetilde{E}_{\varphi}^{M}=W_{M} E_{\varphi}^{M}$. We have

$$
W_{M}[N] \widetilde{E}_{\varphi}^{M}=E_{\varphi}^{M N}+E^{\text {old }}
$$

where the form $E^{\mathrm{old}}$ is weakly old at $N$ (in particular, $E^{\mathrm{old}}$ is orthogonal to newforms of exact level $N$ and so is its product with any other form of level prime to $N$ ).

Proof. It is easy to see that $W_{M}[N] \widetilde{E}_{\varphi}^{M}=[N] E_{\varphi}^{M}$. Then we are reduced to showing

$$
[N] E_{\varphi}^{M}=E_{\varphi}^{M N}+E^{\text {old }} .
$$


In fact, we have more generally and more precisely that

$$
N(N)^{s-} E_{\varphi}^{M}\left(g\left(\begin{array}{cc}
1 & \\
& \pi_{M}
\end{array}\right), s\right)=\sum_{d \mid N} \frac{\varphi(d)}{N(d)^{2 s}} E_{\varphi}^{M N / d}(g, s) ;
$$

this is [Zhang 2001a, Lemma 6.1.4] with $\varepsilon$ replaced by $\varphi$. The lemma then holds with

$$
E^{\mathrm{old}}=\sum_{d \mid N, d \neq 1} \frac{\varphi(d)}{N(d)} E_{\varphi}^{M N / d} .
$$

Lemma 4.3.3. With notation as in Section 4.1, we have

$$
D([\Delta] f, \Theta(\mathfrak{W}), 1)=\mathscr{W}(\mathfrak{D}) D(f, \Theta(\mathbb{W}), 1) .
$$

The proof is as in [Nekovár 1995, §I.5.9].

Theorem 4.3.4. Let $\mathscr{W}: \mathscr{G}^{\prime} \rightarrow \overline{\mathbb{Q}}^{\times}$be a finite-order character of conductor $\mathfrak{f}$ divisible only by primes above $p$. Then we have

$$
L_{p}\left(f_{E}\right)(\mathcal{W})=\frac{\mathscr{W}\left(d_{F}^{(p)}\right) \tau(\bar{W}) N(\Delta(\mathcal{W}))^{1 / 2} V_{p}(f, \mathscr{W})^{\bar{W}}(\Delta)}{\alpha_{\mathfrak{N}(\mathfrak{f}(\mathcal{W}))}(f) \Omega_{f}} L\left(f_{E}, \bar{W}, 1\right),
$$

where $\Omega_{f}=\left(8 \pi^{2}\right)^{g}\langle f, f\rangle_{N}, \tau(\bar{W})$ is as in Lemma 2.4.3 and

$$
V_{p}(f, \bar{W})=\prod_{\wp \mid p} \prod_{\mathfrak{p} \mid \wp}\left(1-\frac{\bar{W}(\mathfrak{p})}{\alpha_{\wp}(f)}\right) .
$$

Proof. Denote $P=\mathfrak{N}(\mathfrak{f}(\mathcal{W})), \Delta(\mathcal{W})=\Delta P$ and $\phi=\left.\mathscr{W}\right|_{F_{A}^{\times}}$. We suppose that $\mathcal{W}$ is ramified at all places $v \mid p$ (in this case, we have $V_{p}(f, \mathscr{W})=1$ ). Then the result follows from the definition and the following calculation:

$$
l_{f_{\alpha}}(\Phi(\mathcal{W}))=\frac{\left\langle W_{N P} f_{\alpha}^{\rho}, \operatorname{Tr}_{\Delta}\left[\Theta(\mathcal{W}) \widetilde{\boldsymbol{E}}_{\varepsilon, N}(\bar{W})\right]\right\rangle_{N P}}{\left\langle W_{N P} f_{\alpha}^{\rho}, f_{\alpha}\right\rangle_{N P}}
$$

Lemma 4.3.1 $=\frac{\left\langle W_{N \Delta} f_{\alpha}^{\rho}, W_{\Delta(\mathcal{W})} \Theta(\mathcal{W}) W_{\Delta(\mathscr{W})} \widetilde{\boldsymbol{E}}_{\varepsilon \phi^{-1}, N}^{\Delta(\mathscr{W})}\right\rangle_{N \Delta(\mathcal{W})}}{\alpha_{P}(f)(-1)^{g} \tau(f) H_{p}(f) \Omega_{f}}$

${ }_{\text {Lemma 2.4.3 }}^{\text {Lemma 4.2 }}=\frac{(-i)^{g \mathscr{W}}\left(d_{F}^{(p)}\right) \tau(\bar{W}) \bar{W}(\mathfrak{D}) D_{E}}{\alpha_{P}(f)(-1)^{g} \tau(f) H_{p}(f) \Omega_{f}}\left\langle W_{N}[\Delta] f_{\alpha}^{\rho}, \Theta(\bar{W}) \boldsymbol{E}_{\varepsilon \phi^{-1}}^{N \Delta(\mathscr{W})}\right\rangle_{N \Delta(\mathbb{W})}$

$$
=\frac{(-i)^{g \mathscr{W}}\left(d_{F}^{(p)}\right) \tau(\bar{W})^{c} \bar{W}(\mathfrak{D})}{\alpha_{P}(f) H_{p}(f) \Omega_{f}}\left\langle[\Delta] f_{\alpha}^{\rho}, \Theta(\bar{W}) \boldsymbol{E}_{\varepsilon \phi^{-1}}^{N \Delta(\mathcal{W}) \bar{W}(\mathfrak{D})}\right\rangle_{N \Delta(\mathcal{W})}
$$

(4.1.1) $=\frac{\mathscr{W}\left(d_{F}^{(p)}\right) \tau(\bar{W}) D_{F}^{2} N(\Delta(\mathcal{W}))^{1 / 2}}{\alpha_{P}(f) H_{p}(f) \Omega_{f}} D^{N \Delta(\mathcal{W})}\left([\Delta] f_{\alpha}, \Theta(\bar{W}), 1\right)$

Lemma 4.3.3 $=\frac{\mathscr{W}\left(d_{F}^{(p)}\right) \tau(\bar{W}) D_{F}^{2} N(\Delta(\mathcal{W}))^{1 / 2} \bar{W}(\Delta)}{\alpha_{P}(f) H_{p}(f) \Omega_{f}} L\left(f_{E}, \bar{W}, 1\right)$, 
where we have used various results from Section 1.4 and the fact that in our case $f^{\rho}=f$ as $f$ has trivial character.

The previous calculation goes through in general with $\Delta(\mathcal{W})$ replaced by $\Delta(\mathcal{W})^{\prime}=$ $\operatorname{lcm}\left(\Delta(\mathcal{W}), \prod_{\wp \mid p} \wp\right)$; then one further needs to compare the imprimitive Dirichlet series $D^{\Delta\left({ }^{(\mathscr{W})^{\prime}}\right.}\left(f_{\alpha}, \Theta(\bar{W}, 1)\right)$ with the $L$-value $L\left(f_{E}, \bar{W}, 1\right)$. This is done in the same way as in the case of elliptic modular forms [Perrin-Riou 1988, Lemme 2.3 (i), $\$ 4.4$ (III)]. ${ }^{27}$ We omit the details since no new phenomena appear in our context and, strictly speaking, we do not need to use the precise form of the interpolation result except in the ramified case (which already determines $L_{p}\left(f_{E}\right)$ uniquely).

4.4. Factorisation. The $p$-adic analogue of the standard $L$-function of $f$ has been studied by several authors (Manin, Dabrowski, Dimitrov, etc.). Let $\mathscr{G}_{F}=$ $\operatorname{Gal}\left(F_{\infty} / F\right)$ where $F_{\infty}$ is the maximal $\mathbb{Z}_{p}$-extension of $F$ unramified outside $p$.

Theorem 4.4.1. There is a p-adic L-function $L_{p}(f) \in \mathfrak{O}_{L} \llbracket \mathscr{G}_{F}^{\prime} \rrbracket \otimes_{\mathcal{O}_{L}} L$ uniquely determined by the following property: for each finite-order character $\chi: \varphi_{F} \rightarrow \overline{\mathbb{Q}}^{\times}$ of conductor $\mathfrak{f}(\chi)$ divisible by all the primes $\wp \mid p$, we have

$$
L_{p}(f, \chi)=\chi\left(d_{F}^{(p)}\right) \frac{\tau(\bar{\chi}) N(\mathfrak{f}(\chi))^{1 / 2}}{\alpha_{\mathfrak{f}(\chi)}} \frac{L(f, \bar{\chi}, 1)}{\Omega_{f}^{+}}
$$

where $\Omega_{f}^{+} \in \mathbb{C}^{\times}$is a suitable period and $\tau(\bar{\chi})=\prod_{v \mid p} \tau\left(\bar{\chi}_{v}\right)$ with

$$
\tau\left(\bar{\chi}_{v}\right)=\left|\pi_{v}\right|^{-c / 2} \int_{F_{v}^{\times}} \bar{W}_{v}\left(x_{v}\right) \boldsymbol{e}_{v}\left(-x_{v}\right) d x_{v}
$$

if $c=v(\mathfrak{f}(\chi))$.

Similarly, we have $L_{p, \varepsilon \alpha}\left(f_{\varepsilon}\right)$ and a period $\Omega_{f_{\varepsilon}}^{+}$satisfying

$$
L_{p, \varepsilon \alpha}\left(f_{\varepsilon}, \chi\right)=\chi\left(d_{F}^{(p)}\right) \frac{\tau(\bar{\chi}) N(\mathfrak{f}(\chi))^{1 / 2}}{\varepsilon(\mathfrak{f}(\chi)) \alpha_{\mathfrak{f}(\chi)}} \frac{L\left(f_{\varepsilon}, \bar{\chi}, 1\right)}{\Omega_{f_{\varepsilon}}^{+}}
$$

for ramified finite-order characters $\chi$. (In fact, $\varepsilon(\mathfrak{f}(\chi)=1$ under our assumptions).)

For the proof of the existence of $L_{p}(f)$, we refer to [Dimitrov 2013]: notice that our $L_{p}(f, \chi)$ equals $\chi\left(d_{F}^{(p)}\right) L_{p}\left(\pi_{f}, \chi^{-1}\right)$ in [op. cit.], where moreover the notation $\tau\left(\chi_{v}\right)$ refers to unnormalised Gauss sums. The definition and properties

${ }^{27}$ Notice that, as in [op. cit.], our $\Theta(\mathcal{W})$ is not the primitive theta series when $\mathcal{W}$ is unramified at some $\wp \mid p$; in general, we have

$$
\Theta(\mathcal{W})=\left(\prod_{\mathfrak{p}|\wp| p}\left(1-N(\wp)^{1 / 2} \mathcal{W}(\mathfrak{p})[\wp]\right)\right) \Theta(W)^{\text {prim }}
$$

if $\Theta(W)^{\text {prim }}$ is the primitive theta series (i.e., the normalised newform in its representation). This replaces the second-to-last equation of [Perrin-Riou 1988, p. 21], whose $\Theta\left({ }^{\prime} W\right)$ and $\Theta\left({ }^{\prime \prime}{ }^{\prime \prime}\right)$ are our $\Theta\left({ }^{\mathcal{W}}\right)^{\text {prim }}$ and $\Theta(\mathcal{W})$, respectively. The factor $V_{p}(f, \bar{W})$ comes from the analogue of [Perrin-Riou 1988, Lemme 23]. 
of the period $\Omega_{f}^{+}$and of a related period $\Omega_{f}^{-}$(both of which are a priori defined up to an $M_{f}^{\times}$-ambiguity) are given in Section 9 below; here we need $\Omega_{f}^{+} \Omega_{f}^{-} \sim \Omega_{f}$ and $\Omega_{f_{\varepsilon}}^{+} \sim D_{E}^{-1 / 2} \Omega_{f}^{-}$, where $\sim$ denotes equality in $\mathbb{C}^{\times} / M_{f}^{\times}$. Then from the complex factorisation $L\left(f_{E}, \chi \circ \mathfrak{N}, s\right)=L(f, \chi, s) L\left(f_{\varepsilon}, \chi, s\right)$ and the interpolation properties satisfied by each factor, we obtain

$$
L_{p}\left(f_{E}, \chi \circ \mathfrak{N}\right)=\chi(\Delta)^{2} \frac{\Omega_{f}^{+} \Omega_{f_{\varepsilon}}^{+}}{D_{E}^{-1 / 2} \Omega_{f}} L_{p}(f, \chi) L_{p}\left(f_{\varepsilon}, \chi\right),
$$

where the period factor is in $M_{f}^{\times}$(in particular, it is algebraic).

4.5. Fourier expansion of the analytic kernel. Consider the restriction of $\Phi$ to $\mathscr{G}$, the Galois group of the maximal $\mathbb{Z}_{p}$-extension of $E$ unramified outside $p$. Any character $\mathscr{W}$ of $\mathscr{G}$ decomposes uniquely as $\mathscr{W}=\mathscr{W}^{+} \mathcal{W}^{-}$with $\left(\mathcal{W}^{+}\right)^{c}=\mathscr{W}$ and $\left(W^{-}\right)^{c}=W^{-1}$ (we say that $\mathscr{W}^{+}$is cyclotomic and $\mathscr{W}^{-}$is anticyclotomic or dihedral). Since we are interested in the case $\varepsilon(N)=(-1)^{g-1}$ in which $\Phi$ is zero on the anticyclotomic characters, we study the restriction of $\Phi$ to the cyclotomic characters. We can write $\mathcal{W}^{+}=\chi \circ \mathfrak{N}$ for a Hecke character $\chi: F^{\times} \backslash F_{\boldsymbol{A}}^{\times} \rightarrow 1+p \mathbb{Z}_{p}$, and we denote

$$
\Theta_{\chi}=\Theta(\chi \circ \mathfrak{N}), \quad \Phi_{\chi}=\Phi(\chi \circ \mathfrak{N}) .
$$

From now on, we assume that $(\Delta, 2)=1$ and all primes $\wp \mid p$ are split in $E$.

Proposition 4.5.1. The Fourier coefficients $b(m)=a_{p}\left(\Phi_{\chi}, m\right)$ of the p-adic modular form $\Phi_{\chi}$ are given by

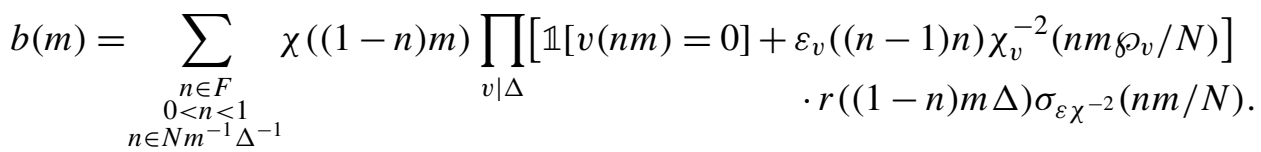

Proof. By (1.4.6), the Fourier coefficient $b(m)$ of $\Phi_{\chi}=\operatorname{Tr}_{\Delta}\left[\Theta_{\chi} \widetilde{\boldsymbol{E}}_{\varepsilon \chi^{2}, N}\right]$ is given by

$$
b(m)=\sum_{\delta \mid \Delta} b^{\delta}(m \delta)
$$

with

$$
\begin{aligned}
b^{\delta}(m)=a\left(\Phi_{\chi}^{(\delta)}, m\right) & =|y|^{-1} \tilde{a}\left(\Phi_{\chi}^{(\delta)}, y\right) \\
& =|y|^{-1} \sum_{n \in F} \tilde{a}\left(\Theta_{\chi}^{(\delta)},(1-n) y\right) \tilde{a}\left(\widetilde{\boldsymbol{E}}_{\varepsilon \chi^{-2}, N}^{(\delta)}, n y\right) \\
& =|y|^{-1} \sum_{n \in F} \tilde{a}\left(\Theta_{\chi}^{(\delta)},(1-n) y\right) \tilde{a}\left(\widetilde{\boldsymbol{E}}_{\varepsilon \chi^{-2}}^{(\delta)}, n y / \pi_{N}\right)
\end{aligned}
$$

if $y \in F_{\boldsymbol{A}}^{\times}$satisfies $y_{\infty}>0$ and $y^{\infty} d_{F}=m$. 
Then by Proposition 2.5.1 and Corollary 3.2.2, we have ${ }^{28}$

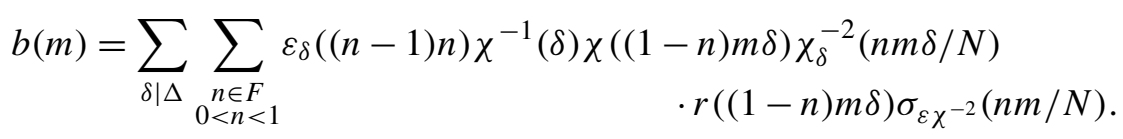

We interchange the two sums and notice that the term corresponding to $\delta$ and $n$ is nonzero only if $n \in N m^{-1} \Delta^{-1}$ and $\delta_{0} \mid \delta$, where

$$
\delta_{0}=\delta_{0}(n)=\prod_{\substack{v \mid \Delta \\ v(n m)=-1}} \wp v
$$

$\left(\wp_{v}\right.$ being the prime corresponding to $v$ ). Now for each $n$, we can rewrite the sum over $\delta$ (omitting the factor $\chi((1-n) m$ ) and those on the second line of (4.5.1), which do not actually depend on $\delta$ ) as

$$
\begin{aligned}
\varepsilon_{\delta_{0}}((n-1) n) \chi_{\delta_{0}}^{-2}\left(n m \delta_{0} / N\right) \sum_{\delta^{\prime} \mid \Delta / \delta_{0}} \varepsilon_{\delta^{\prime}}((n-1) n) \chi_{\delta^{\prime}}^{-2}\left(n m \delta^{\prime} / N\right) \\
=\prod_{v \mid \delta_{0}} \varepsilon_{\delta^{\prime}}((n-1) n) \chi_{v}^{-2}\left(n m \wp_{v}\right) \prod_{v \mid \Delta / \delta_{0}}\left[1+\varepsilon_{v}((n-1) n) \chi_{v}^{-2}\left(n m \wp_{v}\right)\right] .
\end{aligned}
$$

The asserted formula follows.

Remark 4.5.2. If $v(n m)=-1$, then $(n-1) \pi_{m} \pi_{v} \equiv n \pi_{m} \pi_{v}$ in $\left(\mathscr{O}_{F, v} / \pi_{v} \mathcal{O}_{F, v}\right)^{\times}$so that we actually have

$$
\varepsilon_{v}((n-1) n)=\varepsilon_{v}\left((n-1) \pi_{m} \pi_{v}\right) \varepsilon_{v}\left(n \pi_{m} \pi_{v}\right)=1 .
$$

We can now compute the Fourier coefficients of the analytic kernel giving the central derivative of the $p$-adic $L$-function in the cyclotomic direction. To this end, let

$$
v: \operatorname{Gal}(\overline{\mathbb{Q}} / F) \rightarrow 1+p \mathbb{Z}_{p} \subset \mathbb{Q}_{p}^{\times} .
$$

Since $l_{f_{\alpha}}$ is continuous, we have

$$
\frac{d}{d s} L_{p}\left(f_{E}, v^{s} \circ \mathfrak{N}\right)=\frac{d}{d s} l_{f_{\alpha}}(\Phi(s))=l_{f_{\alpha}}\left(\frac{d}{d s} \Phi(s)\right) .
$$

In particular, $L_{p ; \nu \circ \mathfrak{N}}^{\prime}\left(f_{E}, \mathbb{1}\right)=l_{f_{\alpha}}\left(\Phi^{\prime}(0)\right)$.

Let $\ell_{F}=\left.\frac{d}{d s}\right|_{s=0} ^{p ; \nu} \nu^{s}: F^{\times} \backslash F_{A^{\infty}}^{\times} \rightarrow \mathbb{Q}_{p}$ be the $p$-adic logarithm associated with $v$. Proposition 4.5.3. Assume that $\varepsilon(N)=(-1)^{g-1}$. Then $\Phi(0)=0$ and the Fourier coefficients $b^{\prime}(m)$ of

$$
\Phi_{\nu}^{\prime}=\Phi^{\prime}(0)=\left.\frac{d}{d s}\right|_{s=0} \Phi_{v^{s}}
$$

${ }^{28}$ Recall that $\kappa(v)^{2}=\varepsilon_{v}(-1)$. 
are nonzero only for $m$ integral and nonzero, in which case

$$
b^{\prime}(m)=\sum_{v} b_{v}^{\prime}(m)
$$

with the sum running over all finite places $v$ of $F$ and $b_{v}^{\prime}(m)$ given for $\left(\prod_{\wp \mid p} \wp\right) \mid m$ by the following:

(1) If $v=\wp$ is inert in $E$, then

$$
b_{v}^{\prime}(m)=\sum_{\substack{n \in N m^{-1} \Delta^{-1} \\(p, n m)=1 \\ \varepsilon_{v}((n-1) n)=1 \forall v \mid \Delta \\ 0<n<1}} 2^{\omega_{\Delta}(n)} r((1-n) m \Delta) r(n m \Delta / N \wp)(v(n m / N)+1) \ell_{F, v}\left(\pi_{v}\right),
$$

where

$$
\omega_{\Delta}(n)=\#\{v \mid(\Delta, n m \Delta)\} .
$$

(2) If $v=\wp \mid \Delta$ is ramified in $E$, then

$$
b_{v}^{\prime}(m)=\sum_{\substack{\left.\left.n \in N m^{-1} \Delta^{-1} \\(p, n m)=1 \\ \varepsilon_{v}(n-1) n\right)=-1 \\ \varepsilon_{w}(n-1) n\right)=1 \forall v \neq w \mid \Delta \\ 0<n<1}} 2^{\omega_{\Delta}(n)} r((1-n) m \Delta) r(n m \Delta / N)(v(n m)+1) \ell_{F, v}\left(\pi_{v}\right) .
$$

(3) If $v$ is split in $E$, then

$$
b_{v}^{\prime}(m)=0
$$

Proof. The vanishing of $\Phi(0)=\Phi_{\mathbb{1}}$ follows from the functional equation (4.2.4) and the sign assumption.

By Proposition 4.5.1, the Fourier coefficient $b_{s}(m)$ of $\Phi(s)=\Phi_{v^{s}}$ can be expressed as $b_{s}(m)=\sum_{n \in F} b_{n, s}(m)$ with

$$
b_{n, s}(m)=v^{s}((1-n) m) r((1-n) m \Delta) \prod_{v \nmid p \infty} \sigma_{s, v}^{n}(m / N)
$$

where, using Remark 4.5.2,

$$
\sigma_{s, v}^{n}(m)=\left\{\begin{array}{cl}
\frac{1-\varepsilon(n m \wp) v(n m \wp)^{-2 s}}{1-\varepsilon(\wp) v(\wp)^{-2 s}} & \text { if } v=\wp \nmid \Delta, \\
1+\varepsilon_{v}(n(n-1)) v(n m \wp)^{-2 s} & \text { if } v=\wp \mid \Delta \text { and } v(n m)=0, \\
v(n m \wp)^{-2 s} & \text { if } v=\wp \mid \Delta \text { and } v(n m)=-1 .
\end{array}\right.
$$

Then $b^{\prime}(m)=\sum_{n} b_{n}^{\prime}(m)=\sum_{n} \sum_{v} b_{n, v}^{\prime}(m)$ with $\sum_{n} b_{n, v}^{\prime}(m)=b_{v}(m)$, and $b_{n}^{\prime}(m)$ can be nonzero only if exactly one of the factors $\sigma_{s, v}^{n}$ vanishes at $s=0$. If this happens for the place $v_{0}$, then the set over which $n$ ranges accounts for the positivity and integrality conditions and the nonvanishing conditions at other places, whereas the condition $(p, n m)=1$ results from observing that $\lim _{s \rightarrow 0} v^{s}(a)=\mathbb{1}[(p, a)=1]$. 
The values of $b_{n, v}^{\prime}$ can then be determined in each case from the above expressions. For $v$ ramified, this is straightforward. For $v=\wp$ inert, notice that if $v(n m / N)$ is odd then $r(n m \Delta / N \wp)=r\left((n m \Delta / N)^{(\wp)}\right)$, where the superscript denotes primeto- $\wp$ part, whereas if $v(n m / N)$ is even then $\sigma_{0, v}^{n}(m / N)$ does not vanish so $(n, v)$ does not contribute to $b^{\prime}(m)$ and indeed $r(n m / N \wp)=0$.

\section{Part II. Heights}

\section{5. $p$-adic heights and Arakelov theory}

By the work of many authors (Schneider, Perrin-Riou, Mazur and Tate, Coleman and Gross, Zarhin, Nekováŕ, etc.), there are $p$-adic height pairings on the Mordell-Weil group of an abelian variety defined over a number field. In this section, we first recall (Sections 5.1-5.2) a definition of the height pairing as a sum of local symbols following [Zarhin 1990; Nekovár 1993] and explain how it induces a pairing on degree-0 divisors on curves. In Sections 5.3-5.4, we explain how $p$-adic Arakelov theory allows us to extend the height pairing for curves to a pairing on divisors of any degree.

5.1. Localsymbols. Let $A$ be an abelian variety of dimension $g$ over a local field $E_{v}$ and $A^{\vee}$ its dual abelian variety, and let $V=V_{p} A=T_{p} A \otimes_{\mathbb{Z}_{p}} \mathbb{Q}_{p}$ be the rational Tate module of $A$, a continuous $\operatorname{Gal}(\bar{E} / E)$-representation. ${ }^{29}$ Let $\ell_{v}: E_{v}^{\times} \rightarrow \mathbb{Q}_{p}$ be a homomorphism; we call $\ell$ a local $p$-adic logarithm and assume that it is ramified, that is, $\ell_{v}: E_{v}^{\times} \rightarrow \mathbb{Q}_{p}$ does not vanish identically on $\mathcal{O}_{E, v}^{\times}$. Let $D_{\mathrm{dR}}\left(V_{v}\right)$ be the filtered $\mathbb{Q}_{p}$-vector spaces attached to $V_{v}$ by the theory of Fontaine. The comparison theorem identifies $D_{\mathrm{dR}}\left(V_{v}\right)$ with $H_{\mathrm{dR}}^{1}\left(A^{\vee} / E_{v}\right)$, equipped with the Hodge filtration; it is also identified with the filtered Dieudonné module of the special fibre of the $p$-divisible group of $A$ (after an extension of scalars if $E_{v}$ is ramified over $\mathbb{Q}_{p}$ [Fontaine 1982]). Let $L$ be a finite extension of the coefficient field $\mathbb{Q}_{p}$, and if $v \mid p$, let $W_{v} \subset D_{\mathrm{dR}}\left(V_{v}\right) \otimes L$ be a splitting of the Hodge filtration, that is, a complementary subspace to $\Omega^{1}\left(A^{\vee} / E_{v}\right) \otimes L \subset D_{\mathrm{dR}}\left(V_{v}\right) \otimes L$, which is isotropic ${ }^{30}$ for the cup product. When $V_{v}$ is ordinary, there is a canonical choice for $W_{v}$, the "unit root" subspace (see, e.g., [Iovita 2000] for a nice discussion).

We proceed to define pairings, called local Néron symbols, ${ }^{31}$

$$
\langle\cdot, \cdot\rangle_{v, W}:\left(\mathscr{D}_{0}(A)\left(E_{v}\right) \times Z_{0}(A)^{0}\left(E_{v}\right)\right)_{e} \rightarrow L
$$

${ }^{29}$ Nekovář [1993] defines height pairings for Galois representations in much greater generality than described here.

${ }^{30}$ The isotropy condition ensures that the resulting height pairing is symmetric [Nekovár 1993 , Theorem 4.1.1 (4)]

${ }^{31}$ The notation is a bit abusive: the subscript $W$ is meant to recall that the local pairing depends on the choice of $W_{v}$ when $v \mid p$; when $v \nmid p$, it has no meaning. Although the symbol also depends on $\ell$, we will usually omit it from the notation. 
on the subset of pairs with disjoint supports in the product of the group $\mathscr{D}_{0}(A)\left(E_{v}\right)$ of divisors algebraically equivalent to 0 defined over $E_{v}$ and the group $Z_{0}(A)^{0}\left(E_{v}\right)$ of 0 -cycles of degree 0 defined over $E_{v}$.

Let $\mathscr{A} / \mathscr{O}_{E, v}$ and $\mathscr{A}^{\vee} / \mathscr{O}_{E, v}$ be the Néron models of $A$ and $A^{\vee}$, and let $\mathscr{A}^{0}$ be the identity component of $\mathscr{A}$. The rational equivalence class $[D]$ of $D \in \mathscr{D}_{0}(A)\left(E_{v}\right)$ defines a point in $A^{\vee}\left(E_{v}\right)=\mathscr{A}^{\vee}\left(\mathscr{O}_{E, v}\right)=\operatorname{Ext}_{\mathrm{fppf}}^{1}\left(\mathscr{A}^{0}, \boldsymbol{G}_{m}\right)$ and hence an extension

$$
1 \rightarrow \boldsymbol{G}_{m} \rightarrow \mathscr{Y}_{[D]} \rightarrow \mathscr{A}^{0} \rightarrow 1
$$

of abelian fppf sheaves on $\mathscr{O}_{E, v}$, and $\mathscr{Y}_{[D]}$ is represented by a smooth commutative group scheme. On the generic fibre, $\mathscr{Y}_{[D]} \otimes E_{v}$ can be identified with the complement $Y_{D}$ of the zero section in the total space of the line bundle $O(D)$ on $A$, and thus, the extension admits a section

$$
s_{D}: A \backslash|D| \rightarrow Y_{D}
$$

which is canonical up to scaling.

Suppose we are given a morphism $\ell_{v, D, W}$ that makes the following diagram commute:

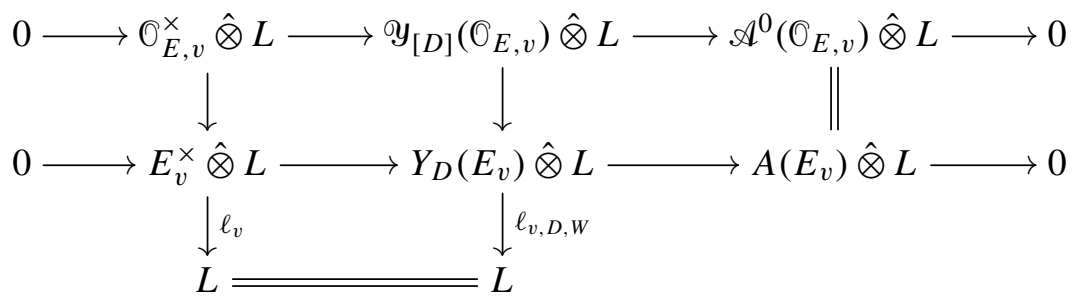

Then we can define the local pairing by

$$
\langle D, z\rangle_{v, W}=\ell_{v, D, W}\left(s_{D}(z)\right),
$$

where $s_{D}$ is extended to the divisor $z$ in the obvious way. Notice that, since $z$ has degree 0 , this is well-defined independently of the scaling ambiguity in $s_{D}$.

When $v \nmid p$, the logarithm $\ell_{v}$ vanishes on $\mathbb{O}_{E, v}^{\times}$for topological reasons and we can uniquely extend it to an $\ell_{v, D}$ as in the above diagram by requiring its restriction to $\mathscr{Y}_{[D]}\left(\mathcal{O}_{E, v}\right)$ to be trivial. When $v \mid p$, given the splitting $W_{v}$, one can construct a section

$$
s_{v, D, W}: A\left(E_{v}\right) \hat{\otimes} L \rightarrow Y_{D}\left(E_{v}\right) \hat{\otimes} L
$$

and define the extension $\ell_{v, D, W}$ by requiring it to be trivial on the image of $s_{v, D, W}$. The standard construction is explained, e.g., in [Kobayashi 2014, §3.2]. In the ordinary case, when $W_{v}$ is chosen to be the unit root subspace, the crucial properties of the (canonical) local symbol are the last two in Proposition 5.1.2 below; in this case, the construction rests on the following result (see [Schneider 1985] or [Nekováŕ 1993, §6.9]): 
Lemma 5.1.1. Let $E_{v, \infty}$ be a totally ramified $\mathbb{Z}_{p}$-extension of $E_{v}$, and denote by $E_{v, n}$ its $n$-th layer. Let $e \in \operatorname{End}(A) \otimes \overline{\mathbb{Q}}$ be an idempotent. Assume that $e V$ is ordinary as a Galois representation. Then the module of universal norms

$$
U\left(e A\left(E_{v}\right)\right)=\bigcap_{n} \operatorname{Im}\left[\operatorname{Tr}_{E_{v, n} / E_{v}}: e A\left(E_{v, n}\right) \rightarrow e A\left(E_{v}\right)\right]
$$

has finite index in eA( $\left.E_{v}\right)$.

Proposition 5.1.2. The p-adic local symbol

$$
\langle\cdot, \cdot\rangle_{v}=\langle\cdot, \cdot\rangle_{v, W}:\left(\mathscr{D}_{0}(A)\left(E_{v}\right) \times Z_{0}(A)^{0}\left(E_{v}\right)\right)_{e} \rightarrow L
$$

defined by (5.1.1) has the following properties (valid whenever they make sense):

(1) It is bilinear.

(2) If $h \in E_{v}(A)$ is a rational function, we have

$$
\langle(h), z\rangle_{v}=\ell_{v}(h(z))
$$

where, if $z=\sum n_{P} P, h(z)=\prod h(P)^{n_{P}}$.

(3) If $\phi: A \rightarrow A$ is a finite endomorphism, we have

$$
\left\langle\phi^{*} D, z\right\rangle_{v}=\left\langle D, \phi_{*} z\right\rangle_{v} .
$$

(4) For any $D \in \mathscr{D}_{0}(A)\left(E_{v}\right)$ and $x_{0} \in A\left(E_{v}\right) \backslash|D|$ the map from $A\left(E_{v}\right) \backslash|D| \rightarrow L$ defined by

$$
x \mapsto\left\langle D, x-x_{0}\right\rangle_{v}
$$

is continuous.

(5) (compatibility) Let $E_{w}^{\prime} / E_{v}$ be a finite extension. If $D \in \mathscr{D}_{0}(A)\left(E_{w}^{\prime}\right)$ and $z \in Z_{0}(A)^{0}\left(E_{v}\right)$, we have

$$
\left\langle\operatorname{Tr}_{E_{w}^{\prime} / E_{v}}\left(D^{\prime}\right), z\right\rangle_{v}=\left\langle D^{\prime}, z\right\rangle_{w}
$$

where $\langle\cdot, \cdot\rangle_{w}$ is the local pairing associated with $\ell_{w}=\ell_{v} \circ N_{E_{w} / E_{v}}$ and (if $\left.v \mid p\right)$ the splitting $W_{w}$ is induced from $W_{v}$.

(6) (boundedness) If $v \mid p$, let $E_{v, \infty}^{\ell}=\bigcup_{n} E_{v, n}^{\ell}$ be the ramified ${ }^{32} \mathbb{Z}_{p}$-extension of $E_{v}$ determined by the isomorphism

$$
E_{v}^{\times} \supset \operatorname{Ker}\left(\ell_{v}\right) \cong \operatorname{Gal}\left(E_{v, \infty}^{\ell} / E\right) \subset \operatorname{Gal}\left(E_{v}^{\mathrm{ab}} / E\right)
$$

induced from class field theory. In the ordinary situation of Lemma 5.1.1, if $e W_{v}$ is the unit root subspace of $e V$, there is a nonzero constant $c \in \mathbb{Z}_{p}$ such that

$$
\langle D, z\rangle_{v, n} \in c^{-1} \ell_{w}\left(E_{v, n}^{\ell, \times}\right)
$$

if $D \in e \mathscr{D}_{0}(A)\left(E_{v, n}\right), z \in e Z_{0}(A)^{0}\left(E_{v, n}\right)$ and $\langle\cdot, \cdot\rangle_{v, n}$ is the local pairing associated with the extension $E_{v, n}^{\ell} / E_{v}$ as in (5).

If $v \nmid p$, the local symbol is characterised by properties (1)-(4).

\footnotetext{
${ }^{32}$ Recall that we choose $\ell_{w}$ to be ramified.
} 
We refer to [Nekovár 1995, §II.1; Kobayashi 2013, §4.2] and references therein for the proof and more details on the construction. See also Proposition 5.4.1 below.

5.2. The p-adic height pairing. Let $A$ be an abelian variety over a number field $E$. Let $\ell: E^{\times} \backslash E_{\boldsymbol{A}}^{\times} \rightarrow \mathbb{Q}_{p}$ be a homomorphism (which we call a global $p$-adic logarithm) whose restrictions $\ell_{v}=\left.\ell\right|_{E_{v}^{\times}}$are ramified for all $v \mid p$. Let $W_{v}$ be Hodge splittings at the places $v \mid p$ as in Section 5.1. Then we can define a height pairing

$$
\langle\cdot, \cdot\rangle: A^{\vee}(E) \times A(E) \rightarrow L
$$

as the sum of local height pairings

$$
\langle x, y\rangle=\sum_{v}\langle\tilde{x}, \tilde{y}\rangle_{v}
$$

where $\tilde{x}$ is a divisor on $A$ whose class in $A^{\vee}(E) \cong \operatorname{Pic}^{0}(A)$ is $x$ and $\tilde{y}=\sum n_{P}[P]$ is a 0 -cycle of degree 0 on $A$ with support disjoint from the support of $\tilde{x}$, which satisfies $\sum n_{P} P=y$. The result is independent of the choices of $\tilde{x}$ and $\tilde{y}$.

Let $X$ be a (proper, smooth) curve over $E$ of genus $g \geq 1$, together with a degree-1 divisor class defined over $E$ inducing an embedding

$$
\iota: X \hookrightarrow J(X)
$$

into its Albanese variety $J(X){ }^{33}$ Let $\operatorname{Div}(X)$ be the group of divisors on $X$, $\operatorname{Div}^{0}(X)$ the subgroup of degree-0 divisors and similarly $\mathrm{CH}(X)=\operatorname{Div}(X) / \sim$ and $\mathrm{CH}(X)_{0}=\operatorname{Div}^{0}(X) / \sim$, the Chow group of 0 -cycles modulo rational equivalence and its subgroup of degree- 0 elements. Then, given a $p$-adic logarithm and Hodge splittings for $V_{p} J(X)$, we can define local and global pairings on degree- 0 divisors on $X$ (denoted with a subscript $X$ ) from the above pairings on $J(X)$ (here denoted with a subscript $J(X))$. Let $D_{1}$ and $D_{2}$ be divisors of degree 0 on $X$ defined over $E$ and with disjoint support. The morphism $\iota$ induces an isomorphism $\iota^{*}: \operatorname{Pic}^{0} J(X) \cong$ $\operatorname{Pic}^{0}(X)$; hence, we can pick an algebraically trivial divisor $D_{1}^{\prime}$ on $J(X)$ satisfying $D_{1}=\iota^{*} D_{1}^{\prime}+(h)$ for some rational function $h \in E(X)$. If $D_{1}^{\prime}$ is chosen so that its support is disjoint from the support of $\iota_{*} D_{2}$ and the support of $(h)$ is disjoint from the support of $D_{2}$, we can define

$$
\left\langle D_{1}, D_{2}\right\rangle_{v, X}=-\left\langle D_{1}^{\prime}, \iota_{*} D_{2}\right\rangle_{v, J(X)}-\ell_{v}\left(h\left(D_{2}\right)\right)
$$

and

$$
\left\langle D_{1}, D_{2}\right\rangle_{X}=\sum_{v}\left\langle D_{1}, D_{2}\right\rangle_{v, X}
$$

${ }^{33}$ In our applications, we only have a rational divisor class, inducing a compatible system of maps $\iota_{E^{\prime}}: X\left(E^{\prime}\right) \otimes \mathbb{Q} \rightarrow J(X)\left(E^{\prime}\right) \otimes \mathbb{Q}$ for $E^{\prime}$ a finite extension of $E$ such that, for some integer $n$, $\left(n \iota^{\prime}\right)_{E^{\prime}}$ is induced from an $E$-morphism. This causes no extra difficulties. 
The latter pairing descends to a height pairing on divisor classes

$$
\langle\cdot, \cdot\rangle: \mathrm{CH}(X)_{0} \times \mathrm{CH}(X)_{0} \rightarrow L .
$$

There are various conventions in the literature for the normalisation of the signs of height pairings. Our choices are the same as those of [Kobayashi 2013, §4.3], whose discussion we have followed and to which we refer for a comparison with other authors' choices.

5.3. p-adic Arakelov theory: local aspects. Here and in Section 5.4, we summarise the main results of Besser [2005], who develops the $p$-adic analogue of classical Arakelov theory.

Metrised line bundles. Let $X_{v}$ be a proper smooth variety over the finite extension $E_{v}$ of $\mathbb{Q}_{p}$, and fix a ramified local $p$-adic logarithm $\ell_{v}: E_{v}^{\times} \rightarrow \mathbb{Q}_{p}$, which we extend to $\overline{\mathbb{Q}}_{p}^{\times}$by $\left.\ell_{v}\right|_{E_{v}^{\prime \times}}=\ell_{v} \circ N_{E_{v}^{\prime} / E_{v}}$ for any finite extension $E_{v}^{\prime} / E_{v}$.

A metrised line bundle $\widehat{\mathscr{L}}=\left(\mathscr{L}, \log _{\mathscr{L}}\right)$ on $X_{v}$ is a line bundle on $X_{v}$ together with a choice of a $\log$ function $\log _{\mathscr{L}}$ on the total space of $\mathscr{L}$ minus the zero section (which will also be viewed as a function on the nonzero sections of $\mathscr{L}$ ). A $\log$ function is the analogue in the $p$-adic theory of the logarithm of a metric on the sections of a line bundle on a Riemann surface. It is a Coleman function having a certain analytic property ${ }^{34}$ and the following algebraic property. If the $p$-adic $\operatorname{logarithm} \ell_{v}$ factors as

$$
\ell_{v}=t_{v} \circ \log _{v}
$$

for some $\log _{v}: E_{v}^{\times} \rightarrow E_{v}$ and some $\mathbb{Q}_{p}$-linear $t_{v}: E_{v} \rightarrow \mathbb{Q}_{p}$, then for any nonzero section $s$ of $\mathscr{L}_{v}$ and rational function $f \in E\left(X_{v}\right)$, we have

$$
\log _{\mathscr{L}, v}(f s)=\log _{v}(f)+\log _{\mathscr{L}, v}(s) .
$$

Adding a constant to a log function produces a new log function; this operation is called scaling.

One can define a notion of $\bar{\partial} \partial$-operator on Coleman functions and attach to any $\log$ function $\log _{\mathscr{L}}$ on $\mathscr{L}$ its curvature $\bar{\partial} \partial \log _{\mathscr{L}} \in H_{\mathrm{dR}}^{1}\left(X_{v}\right) \otimes \Omega^{1}\left(X_{v}\right)$; its cup product is the first Chern class of $\mathscr{L}$.

Log functions on a pair of line bundles induce in the obvious way a log function on their tensor product and similarly for the dual of a line bundle. If $\pi: X_{v} \rightarrow Y_{v}$ is a morphism, then a $\log$ function on a line bundle on $Y_{v}$ induces in the obvious way a $\log$ function on the pullback line bundle on $X_{v}$. If moreover $\pi$ is a finite Galois cover with Galois group $G$ and $\mathscr{L}$ is a line bundle on $X_{v}$ with $\log$ function $\log _{\mathscr{L}}$

${ }^{34}$ For which we refer to [Besser 2005, Definition 4.1]. 
and associated curvature $\beta$, then the norm line bundle $N_{\pi} \mathscr{L}$ on $Y_{v}$ with stalks

$$
\left(N_{\pi} \mathscr{L}\right)_{y}=\bigotimes_{x \mapsto y} \mathscr{L}_{x}^{\otimes e(x \mid y)}
$$

has an obvious candidate $\log$ function $N_{\pi} \log _{\mathscr{L}}$ obtained by tensor product. A delicate point is that it is not automatic that the latter is a genuine $\log$ function (i.e., it satisfies the analytic property alluded to above); see [Besser 2005, Proposition 4.8] for a sufficient condition.

The canonical Green function. Now let $X_{v} / E_{v}$ be a curve of genus $g \geq 1$ with good reduction above $p$. Choose a splitting $W_{v} \subset H_{\mathrm{dR}}^{1}\left(X_{v}\right) \otimes L$ of the Hodge filtration as in Section 5.1, which we use to identify $W_{v} \cong \Omega^{1}\left(X_{v}\right)^{\vee}$; we then define a canonical element

$$
\mu_{X_{v}}=\frac{1}{g} \mathrm{id} \in \text { End } \Omega^{1}\left(X_{v}\right) \cong W_{v} \otimes \Omega^{1}\left(X_{v}\right)
$$

and similarly for the self-product $X_{v} \times X_{v}$ (denoting by $\pi_{1}$ and $\pi_{2}$ the projections)

$$
\Phi=\left(\begin{array}{cc}
1 / g & -1 \\
-1 & 1 / g
\end{array}\right) \in \operatorname{End}\left(\pi_{1}^{*} \Omega^{1}\left(X_{v}\right) \oplus \pi_{2}^{*} \Omega^{1}\left(X_{v}\right)\right) \hookrightarrow H_{\mathrm{dR}}^{1}\left(X_{v} \times X_{v}\right) \otimes \Omega^{1}\left(X_{v} \otimes X_{v}\right) .
$$

The first Chern class of $\Phi$ is the class of the diagonal $\Delta \subset X_{v} \times X_{v}$.

Let $s_{\Delta}$ denote the canonical section of the line bundle $O(\Delta)$ on $X_{v} \times X_{v}$. Given any $\log$ function $\log _{\mathcal{O}(\Delta)}$ on $\mathcal{O}(\Delta)$ with curvature $\Phi$, we can consider the function $G$ on $X_{v} \times X_{v}$ given by

$$
G(P, Q)=\log _{\mathcal{O}(\Delta)}\left(s_{\Delta}\right)(P, Q) .
$$

It is a Coleman function with singularities along $\Delta$; we call $G$ a Green function for $X_{v}$.

A Green function $G$ induces a $\log$ function on any line bundle $\mathcal{O}(D)$ on $X_{v}$ by

$$
\log _{\mathscr{O}(D)}\left(s_{D}\right)(Q)=\sum n_{i} G\left(P_{i}, Q\right)
$$

if $D=\sum n_{i} P_{i}$ and $s_{D}$ is the canonical section of $\mathcal{O}(D)$. A log function $\log _{\mathscr{L}}$ on the line bundle $\mathscr{L}$ and the resulting metrised line bundle $\left(\mathscr{L}, \log _{\mathscr{L}}\right)$ are called admissible with respect to $G$ if, for one (equivalently, any) nonzero rational section $s$ of $\mathscr{L}$, the difference $\log _{\mathscr{L}}(s)-\log _{\operatorname{div}(s)}$ is a constant. Such a constant is denoted by $\iota_{\log }(s)$ or $\iota_{\log _{v}}(s)$ in the case of the trivial line bundle with the log function $\log _{v}$. It is the analogue of the integral of the norm of $s$. It follows easily from the definitions that any isomorphism of admissible metrised line bundles is an isometry up to scaling.

Let $\omega_{X_{v}}$ be the canonical sheaf on $X_{v}$. The canonical isomorphism $\omega_{X_{v}} \cong$ $\Delta^{*} \mathcal{O}(-\Delta)$ gives another way to induce from $G$ a $\log$ function $\log _{\omega_{X_{v}}}^{G}$ on $\omega_{X_{v}}$, namely by pullback (and the resulting metrised line bundle has curvature $(2 g-2) \mu_{X_{v}}$ ). 
The requirement that this log function be admissible, together with a symmetry condition, leads to an almost unique choice of $G$.

Proposition 5.3.1 [Besser 2005, Theorem 5.10]. There exists a unique-up-toconstant symmetric Green function $G$ with associated curvature $\Phi$ such that $\left(\omega_{X_{v}}, \log _{\omega_{X_{v}}}^{G}\right)$ is an admissible metrised line bundle with respect to $G$.

In the following, we will arbitrarily fix the constant implied by the proposition. In our context, the canonical Green function thus determined is, in a suitable sense, defined over $E_{v}$ [Besser 2005, Proposition 8.1].

5.4. p-adic Arakelov theory: global aspects. Let $E$ be a number field with ring of integers $\mathscr{O}_{E}$. Let $\mathscr{X} / \mathcal{O}_{E}$ be an arithmetic surface with generic fibre $X$; that is, $\mathscr{X} \rightarrow \mathcal{O}_{E}$ is a proper regular relative curve and $\mathscr{X} \otimes_{\mathcal{O}_{E}} E=X$. We assume that $\mathscr{X}$ has good reduction at all places $v \mid p$, and denote $X_{v}=\mathscr{Q} \otimes E_{v}$. Fix choices of a ramified $p$-adic logarithm $\ell$ and Hodge splittings $W_{v}$ as in Section 5.3.

Arakelov line bundles and divisors. An Arakelov line bundle on $\mathscr{X}$ is a pair

$$
\widehat{\mathscr{L}}=\left(\mathscr{L},\left(\log _{\mathscr{L}_{v}}\right)_{v \mid p}\right)
$$

consisting of a line bundle $\mathscr{L}$ on $\mathscr{L}$ together with admissible (with respect to the Green functions of Proposition 5.3.1) $\log$ functions $\log _{\mathscr{L}_{v}}$ on $\mathscr{L}_{v}=\left.\mathscr{L}\right|_{X_{v}}$. We denote by $\operatorname{Pic}^{\mathrm{Ar}}(\mathscr{C})$ the group of isometry classes of Arakelov line bundles on $\mathscr{X}$.

The group $\operatorname{Div}^{\mathrm{Ar}}(\mathscr{X})$ of Arakelov divisors on $\mathscr{X}$ is the group of formal combinations

$$
D=D_{\text {fin }}+D_{\infty}
$$

where $D_{\text {fin }}$ is a divisor on $\mathscr{L}$ and $D_{\infty}=\sum_{v \mid p} \lambda_{v} X_{v}$ is a sum with coefficients $\lambda_{v} \in E_{v}$ of formal symbols $X_{v}$ for each place $v \mid p$ of $E$. To an Arakelov line bundle $\widehat{\mathscr{L}}$ and a nonzero rational section $s$ of $\mathscr{L}$, we associate the Arakelov divisor

$$
\widehat{\operatorname{div}}(s)=(s)_{\text {fin }}+(s)_{\infty}
$$

where $(s)_{\text {fin }}$ is the usual divisor of $s$ and $(s)_{\infty}=\sum_{v \mid p} \iota_{\log _{\mathcal{L}_{v}}}\left(s_{v}\right) X_{v}$. The group $\operatorname{Prin}^{\mathrm{Ar}}(\mathscr{X})$ of principal Arakelov divisors on $\mathscr{X}$ is the group generated by the $\widehat{\operatorname{div}}(h)$ for $h \in E(\mathscr{X})^{\times}$. The Arakelov Chow group of $\mathscr{X}$ is

$$
\mathrm{CH}^{\operatorname{Ar}}(\mathscr{X})=\operatorname{Div}^{\operatorname{Ar}}(\mathscr{X}) / \operatorname{Prin}^{\operatorname{Ar}}(\mathscr{X}),
$$

and we have an isomorphism

$$
\operatorname{Pic}^{\mathrm{Ar}}(\mathscr{X}) \cong \mathrm{CH}^{\mathrm{Ar}}(\mathscr{X})
$$

given by $\widehat{\mathscr{L}} \rightarrow[\widehat{\operatorname{div}}(s)]$ for any rational section $s$ of $\mathscr{L}$. 
The p-adic Arakelov pairing. Most important for us is the existence of a pairing on $\mathrm{CH}^{\mathrm{Ar}}(\mathscr{X})$, extending the $p$-adic height pairing of divisors of Section 5.2. Let $(\cdot, \cdot)_{v}$

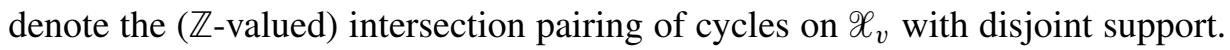

Proposition 5.4.1 [Besser 2005]. Let $\mathscr{X} / \mathscr{O}_{E}$ be an arithmetic surface with good reduction above $p$. For any choice of ramified p-adic logarithm $\ell: E_{A}^{\times} / E^{\times} \rightarrow \mathbb{Q}_{p}$ and Hodge splittings $\left(W_{v}\right)_{v \mid p}$ as above, there is a symmetric bilinear paring ${ }^{35}$

$$
\langle\cdot, \cdot\rangle^{\mathrm{Ar}}: \mathrm{CH}^{\mathrm{Ar}}(\mathscr{X}) \times \mathrm{CH}^{\mathrm{Ar}}(\mathscr{X}) \rightarrow L
$$

satisfying:

(1) If $D_{1}$ and $D_{2}$ are finite and of degree 0 on the generic fibre and one of them has degree 0 on each special fibre of $\mathscr{X}$, then

$$
\left\langle D_{1}, D_{2}\right\rangle^{\mathrm{Ar}}=\left\langle D_{1, E}, D_{2, E}\right\rangle,
$$

where $D_{i, E} \in \operatorname{Div}^{0}(X)$ is the generic fibre of $D_{i}$ and $\langle\cdot, \cdot\rangle$ denotes the height pairing of Proposition 5.1.2 associated with the same choices of $\ell$ and $W_{v}$.

(2) If $D_{1, \text { fin }}$ and $D_{2, \text { fin }}$ have disjoint supports on the generic fibre, then

$$
\left\langle D_{1}, D_{2}\right\rangle^{\mathrm{Ar}}=\sum_{v}\left\langle D_{1}, D_{2}\right\rangle_{v}^{\mathrm{Ar}},
$$

where the sum runs over all finite places of $E$, and the local Arakelov pairings are defined by

$$
\left\langle D_{1}, D_{2}\right\rangle_{v}^{\mathrm{Ar}}=\left(D_{1}, D_{2}\right)_{v} \ell_{v}\left(\pi_{v}\right)
$$

for $v \nmid p$ and below for $v \mid p$.

If moreover we are in the situation of (1), then for each place $v$, we have

$$
\left\langle D_{1}, D_{2}\right\rangle_{v}^{\mathrm{Ar}}=\left\langle D_{1, E}, D_{2, E}\right\rangle_{v} .
$$

(3) In the situation of (2), if moreover $D_{1}=\widehat{\operatorname{div}}(h)$ is the Arakelov divisor of a rational function $h$, then

$$
\left\langle D_{1}, D_{2}\right\rangle_{v}^{\mathrm{Ar}}=\ell_{v}\left(h\left(D_{2, \mathrm{fin}}\right)\right)
$$

for all places $v$.

For completeness, we give the description of the local pairing at $v \mid p$ of divisors with disjoint supports. If $\ell_{v}=t_{v} \circ \log _{v}$ as in (5.3.1) and $G_{v}$ is the Green function on $X_{v} \times X_{v}$, we have $\left\langle D, X_{w}\right\rangle_{v}^{\mathrm{Ar}}=0$ if $v \neq w,\left\langle X_{v}, X_{v}\right\rangle_{v}^{\mathrm{Ar}}=0,\left\langle D, \lambda_{v} X_{v}\right\rangle_{v}^{\mathrm{Ar}}=$ $\left(\operatorname{deg} D_{E}\right) t_{v}\left(\lambda_{v}\right)$ and, if $D_{1}$ and $D_{2}$ are finite divisors with images $D_{1, v}=\sum n_{i} P_{i}$ and $D_{2, v}=\sum m_{j} Q_{j}$ in $X_{v}$,

$$
\left\langle D_{1}, D_{2}\right\rangle_{v}^{\mathrm{Ar}}=\sum_{i, j} n_{i} m_{j} t_{v}\left(G_{v}\left(P_{i}, Q_{j}\right)\right) .
$$

${ }^{35}$ The notation of [Besser 2005] is $D_{1} \cdot D_{2}$ for $\left\langle D_{1}, D_{2}\right\rangle$ Ar. 
In fact, in [Besser 2005], it is proved directly that the global Arakelov pairing and its local components at $p$ coincide with the global and local height pairings of [Coleman and Gross 1989]. The latter coincide with the Zarhin-Nekovár pairings by [Besser 2004].

\section{Heegner points on Shimura curves}

In this section, we describe our Shimura curve and construct Heegner points on it, following [Zhang 2001a, §1-\$2], to which we refer for the details (see also [Zhang 2001b, §5] and [Carayol 1986] for the original source of many results on Shimura curves). We go back to our usual notation, so $F$ is a totally real number field of degree $g, N$ is an ideal of $\mathrm{O}_{F}, E$ is a $\mathrm{CM}$ extension of $F$ of discriminant $\Delta$ coprime to $2 N p$ and $\varepsilon$ is its associated Hecke character.

6.1. Shimura curves. Let $B$ be a quaternion algebra over $F$ that is ramified at all but one infinite place. Then we can choose an isomorphism $B \otimes \mathbb{R} \cong M_{2}(\mathbb{R}) \oplus \boldsymbol{H}^{g-1}$, where $\boldsymbol{H}$ is the division algebra of Hamilton quaternions. There is an action of $B^{\times}$ on $\mathfrak{H}^{ \pm}=\mathbb{C} \backslash \mathbb{R}$ by Möbius transformations via the map $B^{\times} \rightarrow \boldsymbol{G L}_{2}(\mathbb{R})$ induced from the above isomorphism. For each open subgroup $K$ of $\widehat{B}^{\times}=\left(B \otimes_{F} \widehat{F}\right)^{\times}$that is compact modulo $\widehat{F}^{\times}$, we then have a Shimura curve

$$
M_{K}(\mathbb{C})=B^{\times} \backslash \mathfrak{H}^{ \pm} \times \widehat{B}^{\times} / K,
$$

where $\mathfrak{H}^{ \pm}=\mathbb{C} \backslash \mathbb{R}$. Unlike modular curves, the curves $M_{K}$ do not have a natural moduli interpretation. However, by [Carayol 1986], $M_{K}(\mathbb{C})$ has a finite map ${ }^{36}$ to another (unitary) Shimura curve $M_{K^{\prime}}^{\prime}(\mathbb{C})$ that, if the level $K^{\prime}$ is small enough, has an interpretation as the moduli space of certain quaternionic abelian varieties. Namely, $M_{K^{\prime}}^{\prime}$ parametrises isomorphism classes of abelian varieties of dimension $4[F: \mathbb{Q}]$ with multiplication by the ring of integers ${O_{B}}_{B^{\prime}}$ of $B \otimes_{F} F^{\prime}$ and some extra structure (a polarisation and a $K^{\prime}$-level structure, compatible with the quaternionic multiplication) [Zhang 2001a, Proposition 1.1.5].

We will usually denote a point of $M_{K^{\prime}}^{\prime}$ simply by [A], where $A$ is the underlying abelian variety. If $K^{\prime}$ has maximal components at places dividing $m$, one can define a notion of an admissible submodule $D$ of level $m$ [Zhang 2001a, §1.4.3]: it

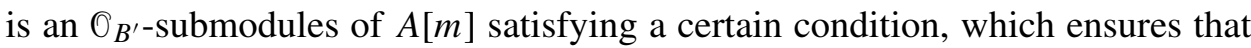
the quotient $A / D$ can be naturally endowed with the extra structure required by the functor $M_{K^{\prime}}^{\prime}$. We denote by $\left[A_{D}\right]$ the object whose underlying abelian variety is $A / D$, with the induced extra structure.

As a consequence of the moduli interpretation, the curve $M_{K}(\mathbb{C})$ has a canonical model $M_{K}$ defined over $F$ (it is connected but not, in general, geometrically

${ }^{36}$ That is an embedding if $K \supset \widehat{F}^{\times}$. 
connected) and a proper regular integral model ${ }^{37} \mu_{K}$ over $\mathscr{O}_{F}$; if $v$ is a finite place where $B$ is split, then $\mu_{K}$ is smooth over $\mathscr{O}_{F, v}$ if $K_{v}$ is a maximal compact subgroup of $B_{v}$ and $K^{v}$ is sufficiently small. We denote $\mu_{K, v}=M_{K} \otimes \mathscr{O}_{F, v}$.

Universal formal group and ordinary points. Assume that the level structure $K$ is maximal at $\wp$. The curve $\mathcal{M}_{K, \wp}$ carries a universal $\wp$-divisible $\mathscr{O}_{B, \wp}$-module $\varphi$ obtained from the $\wp$-divisible group $\mathscr{A}\left[\wp^{\infty}\right]$ of the universal abelian scheme $\mathscr{A}$ over $M_{K^{\prime}, \wp}^{\prime}$. More precisely, choosing an auxiliary quadratic field $F^{\prime}$ that is split at $\wp$ and an isomorphism $j: \mathscr{O}_{F^{\prime}, \wp} \cong \mathscr{O}_{F, \wp} \oplus \mathcal{O}_{F, \wp}$, we have

$$
\varphi=\mathscr{A}\left[\wp^{\infty}\right]^{(2)}=e_{2} \mathscr{A}\left[\wp^{\infty}\right],
$$

where $e_{2}$ is the idempotent in $\mathscr{O}_{F^{\prime}, \wp}$ corresponding to $(0,1)$ under $j$.

Assume that $B$ is split at $\wp$. Then we denote by $\varphi^{1}$ and $\varphi^{2}$ the images under the projectors corresponding to $\left(\begin{array}{cc}1 & \\ & 0\end{array}\right)$ and $\left(\begin{array}{c}0 \\ { }_{1}\end{array}\right)$ under a fixed isomorphism $B \cong M_{2}\left(F_{\wp}\right)$; they are isomorphic via the element $\left({ }_{-1}^{1}\right)$.

Let $x$ be a geometric point of the special fibre $\mu_{K, \wp}$. Then the $\varphi_{x}^{i}$ are divisible $\mathrm{O}_{\wp}$-modules of dimension 1 and height 2 and hence isomorphic to either

- the direct sum $\Sigma_{1} \oplus F_{\wp} / \mathcal{O}_{F, \wp}$, where $\Sigma_{1}$ is the unique formal $\mathscr{O}_{F, \wp}$-module of height 1 - in this case, $x$ is called ordinary - or

- the unique formal $O_{\wp}$-module of dimension 1 and height 2 - in this case, $x$ is called supersingular.

Let $M_{K}\left(\overline{\mathbb{Q}}_{\wp}\right)^{\text {ord }} \subset M_{K}\left(\overline{\mathbb{Q}}_{\wp}\right)$ be the set of points with ordinary reduction. Then the Frobenius map Frob

$$
\varphi: M_{K}\left(\bar{F}_{\wp}\right)^{\text {ord }} \rightarrow M_{K}\left(\bar{F}_{\wp}\right)^{\text {ord }}
$$

given in the moduli interpretation by $[A] \mapsto\left[A_{\operatorname{can}(A)}\right]$, where can $(A)$ is the canonical submodule of $A$, that is, the sub- $O_{F, \wp}$-module of $A[\wp]$ in the kernel of multiplication by $\wp$ in the formal group of $A$.

The order $R$ and the curve $X$. Assume that $\varepsilon(N)=(-1)^{g-1}$. Then the quaternion algebra $\boldsymbol{B}$ over $\boldsymbol{A}_{F}$ ramified exactly at all the infinite places and the finite places $v \mid N$ such that $\varepsilon(v)=-1$ is incoherent; that is, it does not arise via extension of scalars from a quaternion algebra over $F$. On the other hand, for any embedding $\tau: F \hookrightarrow \mathbb{R}$, there is a nearby quaternion algebra $B(\tau)$ defined over $F$ and ramified at $\tau$ and the places where $\boldsymbol{B}$ is ramified. Fix any embedding $\rho: E \rightarrow B(\tau)$, and let $R$ be an order of $\widehat{B}=\widehat{B}(\tau)$ that contains $\rho\left(\mathcal{O}_{E}\right)$ and has discriminant $N$ (this is constructed in [Zhang 2001a, §1.5.1]). Then the curve $X$ over $F$ of interest to us is the

${ }^{37}$ In the modular curve case $F=\mathbb{Q}, \varepsilon(v)=1$ for all $v \mid N, M_{K}$ and $M_{K}$ are proper only after the addition of finitely many cusps. (We caution the reader that [Carayol 1986] uses the notation $\mu_{K}$ to denote instead the set of geometrically connected components of $M_{K}$.) 
(compactification of) the curve $M_{K}$ defined above for the subgroup $K=\widehat{F}^{\times} \widehat{R}^{\times} \subset \widehat{B}$; that is, for each embedding $\tau: F \rightarrow \mathbb{C}$, we have

$$
X(\mathbb{C})=B(\tau)^{\times} \backslash \mathfrak{H}^{ \pm} \times \widehat{B}^{\times} / \widehat{F}^{\times} \widehat{R}^{\times} \cup\{\text { cusps }\} .
$$

The finite set of cusps is nonempty only in the classical case where $F=\mathbb{Q}$ and $\varepsilon(v)=1$ for all $v \mid N$ so that $X=X_{0}(N)$. In what follows, we will not burden the notation with the details of this particular case, which poses no additional difficulties and is already treated in the original work of Perrin-Riou [1987].

We denote by $\mathscr{X}$ the canonical model of $X$ over $\mathscr{O}_{F}$ and by $\mathscr{L}_{v}$ its base change to $\mathscr{O}_{F, v}$. We also denote by $J(X)$ the Albanese variety of $X$ and by $\mathscr{g}_{v}$ its Néron model over $\mathfrak{O}_{F, v}$.

Hecke correspondences. Let $m$ be an ideal of $\mathbb{O}_{F}$ that is coprime to the ramification set of $B$. Let $\gamma_{m} \in \hat{O}_{B}$ be an element with components 1 away from $m$ and such that det $\gamma_{m}$ generates $m$ at the places dividing $m$. Then the Hecke operator $T(m)$ on $X$ is defined by

$$
T(m)[(z, g)]=\sum_{\gamma \in K \gamma_{m} K / K}[(z, g \gamma)]
$$

under the complex description (6.1.2). When $m$ divides $N$, we often denote the operator $T(m)$ by $U(m)$ or $U_{m}$.

Let $\boldsymbol{T}_{N}^{\prime}$ be the algebra generated by the $T(m)$ for $m$ prime to $N$. Then by [Zhang 2001a, Theorem 3.2.1], the algebra $\boldsymbol{T}_{N}^{\prime}$ is a quotient of the Hecke algebra on Hilbert modular forms $\boldsymbol{T}_{N}$ (hence, the names $T(m)$ are justified). It acts by correspondences on $X \times X$, and taking Zariski closures of cycles on $\mathscr{X} \times \mathscr{X}$ extends the action to $\mathscr{X}$.

As in the classical case, the Hecke operators $T(m)$ admit a moduli interpretation, after base change to a suitable quadratic extension $F^{\prime}$ and passing to the curve $X^{\prime}$. Namely we have

$$
T(m)[A]=\sum_{D}\left[A_{D}\right]
$$

where the sum runs over the admissible submodules of $A$ of level $m$.

6.2. Heegner points. The curve $X$ defined above has a distinguished collection of points defined over abelian extensions of $E$ : we briefly describe it, referring the reader to [Zhang 2001a, §2] for more details.

A point $y$ of $X$ is called a $C M$ point with multiplication by $E$ if it can be represented by $\left(x_{0}, g\right) \in \mathfrak{H}^{+} \times \widehat{B}^{\times}$via (6.1.2), where $x_{0} \in \mathfrak{H}^{+}$is the unique point fixed by $E^{\times}$. The order

$$
\operatorname{End}(y)=g \widehat{R} g^{-1} \cap \rho(E)
$$


in $E=\rho(E)$ is defined independently of the choice of $g$, and

$$
\operatorname{End}(y)=\mathrm{O}_{E}[c]=\mathscr{O}_{F}+c \mathcal{O}_{E}
$$

for a unique ideal $c$ of $\mathfrak{O}_{F}$ called the conductor of $y$. We say that the point $y=\left[\left(x_{0}, g\right)\right]$ has the positive orientation if for every finite place $v$ the morphism $t \mapsto g^{-1} \rho(t) g$ is $R_{v}^{\times}$-conjugate to $\rho$ in $\operatorname{Hom}\left(\mathcal{O}_{E, v}, R_{v}\right) / R_{v}^{\times} .{ }^{38}$ Let $Y_{c}$ be the set of positively oriented CM points of conductor $c$. By the work of Shimura and Taniyama, it is a finite subscheme of $X$ defined over $E$, and the action of $\operatorname{Gal}(\overline{\mathbb{Q}} / E)$ is given by

$$
\sigma\left[\left(x_{0}, g\right)\right]=\left[\left(x_{0}, \operatorname{rec}_{E}(\sigma) g\right)\right],
$$

where $\operatorname{rec}_{E}: \operatorname{Gal}(\bar{E} / E) \rightarrow \operatorname{Gal}(\bar{E} / E)^{\text {ab }} \stackrel{\sim}{\rightarrow} \overline{E^{\times}} \backslash \widehat{E}^{\times}$is the reciprocity map of class field theory. If $y=\left[\left(x_{0}, g\right)\right]$ has conductor $c$, then the action factors through

$$
\operatorname{Gal}(H[c] / E) \cong E^{\times} \backslash \widehat{E}^{\times} / \widehat{F}^{\times} \hat{\mathrm{O}}_{E}[c]^{\times},
$$

where $H[c]$ is the ring class field of $E$ of conductor $c$; the action of this group on $Y_{c}$ is simply transitive.

For each nonzero ideal $c$ of $\mathscr{O}_{F}$, let $u(c)=\left[\mathscr{O}_{E}[c]^{\times}: \mathscr{O}_{F}^{\times}\right]$and define the divisor

$$
\eta_{c}=u(c)^{-1} \sum_{y \in Y_{c}} y .
$$

Let $\eta=\eta_{1}$. By the above description of the Galois action on CM points, each divisor $\eta_{c}$ is defined over $E$.

A Heegner point $y \in X(H)$ is a positively oriented CM point with conductor 1 . We can use the embedding $\iota: X \rightarrow J(X) \otimes \mathbb{Q}$ to define the point

$$
[z]=\iota(\eta)=[\eta]-h[\xi] \in J(X)(E) \otimes \mathbb{Q},
$$

where $h$ is a number such that $[z]$ has degree 0 in each geometrically connected component of $X$ and $[\xi]$ is the Hodge class of the introduction (see below for more on the Hodge class).

Arakelov Heegner divisors. The Heegner divisor on $X$ can be refined to an Arakelov divisor $\hat{z}$ having degree 0 on each irreducible component of each special fibre. On a suitable Shimura curve $\widetilde{X} \stackrel{\pi}{\rightarrow} X$ of deeper level away from $N \Delta_{E / F}$, we can give an explicit description of the pullback $\hat{\tilde{z}}$ of $\hat{z}$ and of the Hodge class as follows.

As outlined in Section 6.1, after base change to a suitable quadratic extension $F^{\prime}$ of $F$, we have an embedding $\widetilde{X} \hookrightarrow \widetilde{X}^{\prime}$ of $\widetilde{X}=M_{\widetilde{K}}$ into the unitary Shimura curve $\tilde{X}^{\prime}=M_{\widetilde{K}^{\prime}}^{\prime}$ parametrising abelian varieties of dimension $4 g$ with multiplication

${ }^{38}$ This set has two elements only if $v \mid N$ (the other element is called the negative orientation at $v$ ); otherwise, it has one element and the condition at $v$ is empty. There is a group of Atkin-Lehner involutions acting transitively on orientation classes. 
by $\hat{O}_{B^{\prime}}$ and some extra structure. Then by the Kodaira-Spencer map, we have an isomorphism $\omega_{\widetilde{X}^{\prime}} \cong \operatorname{det}$ Lie $\left.\mathscr{A}^{\vee}\right|_{\widetilde{X}^{\prime}}$, where $\mathscr{A} \rightarrow \widetilde{X}^{\prime}$ is the universal abelian scheme and the determinant is that of an $O_{F^{\prime}}$-module of rank 4 (the structure of $O_{F^{\prime}}$-module coming from the multiplication by $\widehat{O}_{B^{\prime}}$ on $\mathscr{A}$ ). This gives a way ${ }^{39}$ of extending the line bundle $\omega_{\widetilde{X}^{\prime}}$ to the integral model $\widetilde{\mathscr{X}}^{\prime}$ and to a line bundle $\mathscr{L}$ on $\widetilde{\mathscr{L}}$. For each finite place $v \mid p$, we endow $\left.\mathscr{L}\right|_{\widetilde{X}_{v}}$ with the canonical $\log$ functions $\log _{\mathscr{L}, v}$ coming from the description $\left.\mathscr{L}\right|_{\widetilde{X}_{v}}=\omega_{\widetilde{X}_{v}}$ and a fixed choice of Hodge splittings on $\widetilde{X}$. We define $[\hat{\xi}] \in \mathrm{CH}^{\mathrm{Ar}}(\widetilde{\mathscr{X}}) \otimes \mathbb{Q}$ to be the class of $\left(\mathscr{L},\left(\log _{\mathscr{L}}\right)_{v \mid p}\right)$ divided by its degree, $[\tilde{\xi}]$ to be its finite part and $\hat{\xi}$ to be any Arakelov divisor in its class.

Then the Arakelov Heegner divisor $\hat{\tilde{z}} \in \operatorname{Div}^{\operatorname{Ar}}\left(\mathscr{X} \otimes \mathcal{O}_{E}\right)$ is described by

$$
\hat{\tilde{z}}=\hat{\tilde{\eta}}-h \hat{\tilde{\xi}}+Z
$$

where $\hat{\tilde{\eta}}$ is the Zariski closure in $\mathscr{X} \otimes \mathcal{O}_{E}$ of the pullback of $\eta$ to $\tilde{X}$ and $Z$ is a finite vertical divisor uniquely determined by the requirement that $\hat{\tilde{z}}$ should have degree 0 on each irreducible component of each special fibre.

6.3. Hecke action on Heegner points. Recall from Section 1.5 the spaces of Fourier coefficients $\mathscr{D}_{N} \subset \mathscr{Y}$, the arithmetic functions $\sigma_{1}, r \in \mathscr{D}_{N}$ and the space $\overline{\mathscr{Y}}=\mathscr{Y} / \mathscr{D}_{N}$. The action of Hecke operators on the Arakelov Heegner divisor is described as follows.

Proposition 6.3.1. Let $m$ be an ideal of $O_{F}$ coprime to $N$. We have:

(1) $T(m) \eta=\sum_{c \mid m} r(m / c) \eta_{c}$.

(2) Let $\eta_{c}^{0}=\sum_{\mathscr{O}_{F} \neq d \mid c} \eta_{d}$, and let $T^{0}(m) \eta=\sum_{c \mid m} \varepsilon(c) \eta_{m / c^{0}}^{0}$. Then $\eta$ and $T^{0}(m) \eta$ have disjoint support, and if $m$ is prime to $N \Delta$, then $T(m) \eta=T^{0}(m) \eta+r(m) \eta$.

(3) $T(m)[\xi]=\sigma_{1}(m)[\xi]$, and $m \mapsto T(m) \hat{\tilde{\xi}}$ is zero in $\overline{\mathscr{S}} \otimes \operatorname{Div}^{\operatorname{Ar}}(\widetilde{\mathscr{X}})$.

(4) The arithmetic function $m \mapsto T(m) Z$ is zero in $\overline{\mathscr{S}} \otimes \operatorname{Div}^{\mathrm{Ar}}(\mathscr{X})$.

Proof. Parts (1), (2) and (4) are proved in [Zhang 2001a, §4]. For part (3), we switch to the curve $\widetilde{X}$. By definition, $[\hat{\tilde{\xi}}]$ is a multiple of the class of the Arekelov line bundle $\mathscr{L}=\operatorname{det} \operatorname{Lie} \mathscr{A}^{\vee}$ on $\widetilde{\mathscr{L}}$ with the canonical log functions on $\mathscr{L}_{v} \cong \omega_{\widetilde{X}_{v}}$, where $\mathscr{A} \rightarrow \mathscr{X}$ is the universal abelian scheme. We view $T(m)$ as a finite algebraic correspondence of degree $\sigma_{1}(m)$ induced by the subscheme $\widetilde{\mathscr{X}}_{m} \subset \widetilde{\mathscr{X}} \times \widetilde{\mathscr{X}}$ of pairs $(A, A / D)$ where $D$ is an admissible submodule of $A$ of level $m$. If $p_{1}, p_{2}: \widetilde{\mathscr{x}}_{m} \rightarrow \widetilde{\mathscr{X}}$ are the two projections, then we have

$$
T(m) \mathscr{L}=N_{p_{1}} p_{2}^{*} \mathscr{L},
$$

${ }^{39}$ See [Zhang 2001a, §4.1.3, §1] for more details on this construction. 
and the $\log$ functions $\log _{T(m) \mathscr{L}}$ on $\left.T(m) \mathscr{L}\right|_{\widetilde{X}_{v}}$ are the ones induced by this description. (That these are genuine log functions - see the caveat in Section 5.3 - will be shown in the course of proving Proposition 6.3.1(3) below.)

Let $\pi: \mathscr{A}_{1} \rightarrow \mathscr{A}_{2}$ be the universal isogeny over $\widetilde{\mathscr{X}}_{m}$. As $p_{i}^{*} \mathscr{L}=\operatorname{det} \operatorname{Lie} \mathscr{A}_{i}^{\vee}$, we have an induced map

$$
\psi_{m}=N_{p_{1}} \pi^{*}: T(m) \mathscr{L} \rightarrow N_{p_{1}} p_{1}^{*} \mathscr{L}=\mathscr{L}^{\sigma_{1}(m)},
$$

and [Zhang 2001a, §4.3] shows that $\psi_{m}(T(m) \mathscr{L})=c_{m} \mathscr{L}^{\sigma_{1}(m)}$ where $c_{m} \subset \mathscr{O}_{F}$ is an ideal with divisor $\left[c_{m}\right]$ on Spec $O_{F}$ such that $m \rightarrow\left[c_{m}\right]$ is a $\sigma_{1}$-derivative (Section 1.5) and hence zero in $\overline{\mathscr{S}} \otimes \operatorname{Div}\left(\operatorname{Spec} \mathscr{O}_{F}\right) \subset \overline{\mathscr{S}} \otimes \operatorname{Div}^{\operatorname{Ar}}(\mathscr{X})$. In fact if the finite divisor $\hat{\tilde{\xi}}_{\text {fin }}=\operatorname{div}(s)$ for a rational section $s$ of $\mathscr{L}$, the same argument shows that $T(m) \hat{\tilde{\xi}}_{\text {fin }}=\operatorname{div}(T(m) s)=\sigma_{1}(m) \operatorname{div}(s)+\operatorname{div}\left(c_{m}\right)$; hence, $m \mapsto \hat{\tilde{\xi}}_{\text {fin }}$ is zero in $\overline{\mathscr{S}} \otimes \operatorname{Div}^{\mathrm{Ar}}(\mathscr{X})$.

We complete the proof by showing that, for each $v \mid p$, the difference of log functions

$$
\psi_{m}^{*} \log _{\mathscr{L}_{v}^{\sigma_{1}(m)}}-\log _{T(m) \mathscr{L}_{v}}
$$

on the line bundle $T(m) \mathscr{L}_{v}$ on $\widetilde{X}_{v}$ is a constant on the total space of $\mathscr{L}_{v}$, and it is a $\sigma_{1}$-derivative when viewed as a function of $m$. (In particular, this shows that $\log _{T(m) \mathscr{L}_{v}}=\sigma_{1}(m) \psi_{m}^{*} \log _{\mathscr{L}_{v}}+$ constant is a genuine $\log$ function.)

It is enough to show this after pullback via $p_{1}$ on $\widetilde{X}_{m}$, where (denoting pulledback objects with a prime) the map $\psi_{m}^{\prime}$ decomposes as

$$
\psi_{m}^{\prime}=\bigotimes_{D} \pi_{D}^{*}: \bigotimes_{D} \operatorname{det} \operatorname{Lie}\left(\mathscr{A}^{\prime} / D\right)^{\vee} \rightarrow\left(\operatorname{det} \operatorname{Lie} \mathscr{A}^{\vee \vee}\right)^{\otimes \sigma_{1}(m)},
$$

where the tensor product runs over admissible submodule schemes of level $m$ of $\mathscr{A l}^{\prime}$ (since base change via $p_{1}$ splits the cover $p_{1}$, there are exactly $\sigma_{1}(m)$ of those). Now the difference (6.3.1) is the sum of the $\sigma_{1}(m)$ differences

$$
\left(\pi_{D}^{*}\right)^{*} \log _{\mathscr{L}}-\log _{\mathscr{L}},
$$

which are all the same since they are permuted by the Galois group of $p_{1}$. As $\pi_{D}^{*}$ acts by multiplication by $\left(\operatorname{deg} \pi_{D}\right)^{1 / 2}=N(m)^{2}$, by (5.3.2), each of these differences is $2 \log _{v} \boldsymbol{N}(m)$ so that (6.3.1) equals

$$
2 \sigma_{1}(m) \log _{v} N(m),
$$

which is indeed a $\sigma_{1}$-derivative.

\section{Heights of Heegner points}

Let $\Psi$ be the modular form of level $N$ with Fourier coefficients given by the $p$-adic height pairing $\langle z, T(m) z\rangle$ (it is a modular form because of Lemma 1.4.1 and the 
fact that the quaternionic Hecke algebra $\boldsymbol{T}_{N}^{\prime}$ is a quotient of $\boldsymbol{T}_{N}$, as explained at the end of Section 6.1). We will compute the heights of Heegner points, with the goal of showing (in Section 8) that $l_{f_{\alpha}}\left(\Phi^{\prime}\right)$ and $l_{f_{\alpha}}(\Psi)$ are equal up to the action of some Hecke operators. The main theorem will follow.

The strategy is close to that of Perrin-Riou. Namely, we separate the local contributions to $\Psi$ from primes above $p$, writing $\Psi \sim \Psi_{\text {fin }}+\Psi_{p}$; using the computations of [Zhang 2001a; 2001b], we find an explicit expression for $\Psi_{\text {fin }}$, which in Section 8 we will show to be "almost" equal to the expression for $\Phi^{\prime}$, while the contribution of $\Psi_{p}$ is shown to vanish. We circumvent the difficulties posed by the absence of cusps through the use of $p$-adic Arakelov theory.

It will be crucial to work in the quotient spaces $\overline{\mathscr{S}}$ and $\overline{\mathscr{S}}^{\text {ord }}$ introduced in Section 1.6; for the convenience of the reader, we copy here the diagram (1.6.1) that summarises the relations among them.

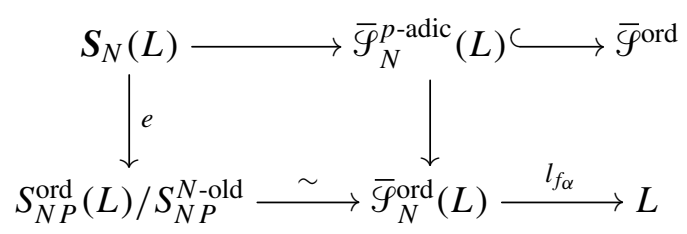

We will abuse notation by using the same name for a modular form and its image in $\overline{\mathscr{S}_{N}}$ ord .

The height pairings $\langle\cdot, \cdot\rangle$ (and the accompanying Arakelov pairings) on the base change of $X$ to $E$ that will be considered are the ones associated with a "cyclotomic" $p$-adic logarithm given by $\ell=\ell_{F} \circ \mathfrak{N}: E^{\times} \backslash E_{A^{\infty}}^{\times} \rightarrow \mathbb{Q}_{p}$ for some ${ }^{40}$

$$
\ell_{F}: F^{\times} \backslash F_{A^{\infty}}^{\times} \rightarrow \mathbb{Q}_{p}
$$

and with choices of Hodge splittings on $V_{v, L}=H_{\mathrm{dR}}^{1}\left(X_{v} / E_{v}\right) \otimes L(v \mid p)$ such that, on $e_{f} V_{v, L} \cong e_{f} M_{\mathscr{F}, L}$, the induced Hodge splitting is the unit root splitting.

As mentioned before, the Shimura curve $X$ and its integral model $\mathscr{L}$ may not be fine enough for the needs of Arakelov and intersection theory, so we may need to pass to a Shimura curve $\widetilde{\mathscr{X}} \stackrel{\pi}{\rightarrow} \mathscr{\mathscr { C }}$ of deeper level away from $p$ and consider the pullbacks $\tilde{\eta}$ of the divisors $\eta$, etc. Then notation such as $\left\langle\hat{\eta}, T^{0}(m) \hat{\eta}\right\rangle^{\mathrm{Ar}}$ is to be properly understood as $\left\langle\hat{\tilde{\eta}}, T^{0}(m) \hat{\tilde{\eta}}\right\rangle^{\mathrm{Ar}} / \operatorname{deg} \pi$.

7.1. Local heights at places not dividing p. The next two results will be used to show the main identity.

Lemma 7.1.1. In the space $\bar{Y}$ we have

$$
\langle z, T(m) z\rangle=\langle\hat{z}, T(m) \hat{z}\rangle^{\mathrm{Ar}} \sim\left\langle\hat{\eta}, T^{0}(m) \hat{\eta}\right\rangle^{\mathrm{Ar}} .
$$

${ }^{40}$ In our application, we will take $\ell_{F}=\left.\frac{d}{d s}\right|_{s=0} \nu^{s}$ for a character $v: \varphi_{F} \rightarrow 1+p \mathbb{Z}_{p}$. 
Proof. First observe that, by Lemma 1.4.1, the first member is a modular form of level $N$, so it does indeed belong to $\overline{\mathscr{S}}_{N}$. The first equality is a consequence of Proposition 5.4.1(1) and the construction of $\hat{z}$. The second part follows from expanding the second term for $m$ prime to $N \Delta$ according to (6.2.2) and observing that the omitted terms are zero in $\overline{\mathscr{Y}}$ by Proposition 6.3.1.

We can therefore write

$$
\Psi \sim \sum_{w} \Psi_{w}=\sum_{v} \Psi_{v}=\Psi_{\text {fin }}+\Psi_{p}
$$

in $\overline{\mathscr{Y}}$, with the first sum running over the finite places $w$ of $E$, the second sum running over the finite places $v$ of $F$ and

$$
\Psi_{w}(m)=\left\langle\hat{\eta}, T^{0}(m) \hat{\eta}\right\rangle_{w}^{\mathrm{Ar}}, \quad \Psi_{v}=\sum_{w \mid v} \Psi_{w}, \quad \Psi_{\mathrm{fin}}=\sum_{v \nmid p} \Psi_{v}, \quad \Psi_{p}=\sum_{v \mid p} \Psi_{v} .
$$

(We are exploiting the fact that for $m$ prime to $N \Delta$ the divisors $\hat{\eta}$ and $T^{0}(m) \hat{\eta}$ have disjoint supports so that we can apply Proposition 5.4.1(2).)

For each prime $\wp$ of $F$ above $p$, we define an operator ${ }^{41}$ on $\mathscr{S}$

$$
\mathscr{R}_{\wp}=U_{\wp}-1, \quad \mathscr{R}_{p}=\prod_{\wp \mid p} \mathscr{R}_{\wp} .
$$

We also define, for integers $\mu_{\wp} \geq 1$, operators

$$
\mathscr{R}_{\wp}^{\left(\mu_{\wp}\right)}=U_{\wp}^{\mu_{\wp}}-1, \quad \mathscr{R}_{p}^{(\mu)}=\prod_{\wp \mid p} \mathscr{R}_{\wp}^{\left(\mu_{\wp}\right)} .
$$

Proposition 7.1.2. In the space $\overline{\mathscr{Y}}$, we have

$$
\Psi_{\text {fin }} \sim \sum_{v \nmid p} \Psi_{v}+h
$$

where $h$ is a modular form that is killed by $l_{f_{\alpha}}$; the sum runs over the finite places of $F$, and the summands are given by:

(1) If $v=\wp$ is inert in $E$, then

$$
\Psi_{v}(m)=\sum_{\substack{n \in N m^{-1} \Delta^{-1} \\ \varepsilon_{v}((n-1) n)=1 \forall v \mid \Delta \\ 0<n<1}} 2^{\omega_{\Delta}(n)} r((1-n) m \Delta) r(n m \Delta / N \wp)(v(n m / N)+1) \ell_{F, v}\left(\pi_{v}\right) .
$$

${ }^{41}$ This is different from the operator bearing the same name in [Perrin-Riou 1987]. 
(2) If $v=\wp \mid \Delta$ is ramified in $E$, then

$$
\Psi_{v}(m)=\sum_{\substack{n \in N m^{-1} \Delta^{-1} \\ \varepsilon_{v}((n-1) n)=-1 \\ \varepsilon_{w}((n-1) n)=1 \forall v \neq w \mid \Delta \\ 0<n<1}} 2^{\omega_{\Delta}(n)} r((1-n) m \Delta) r(n m \Delta / N)(v(n m)+1) \ell_{v}\left(\pi_{v}\right) .
$$

(3) If $v$ is split in $E$, then

$$
\Psi_{v}(m)=0
$$

Proof. For $m$ prime to $N \Delta$, we have $\Psi_{\text {fin }}(m)=\sum_{w \nmid p}\left\langle\hat{\eta}, T^{0}(m) \hat{\eta}\right\rangle_{w}^{\mathrm{Ar}}$ (the sum running over all finite places $w$ of $E$ ). By Proposition 5.4.1(2), up to the factor $\ell_{F, v}\left(\pi_{v}\right)$ (which equals $\ell_{w}\left(\pi_{w}\right)$ or its half for each place $w$ of $E$ above $v$ ), each term is given by an intersection multiplicity $(\hat{\eta}, T(m) \hat{\eta})_{w}$, which is computed by Zhang.

When $v(N) \leq 1$ for all $v$ that are not split in $E$, the result is summarised in [Zhang 2001a, Proposition 5.4.8]; in this case, the values obtained there are equivalent to the asserted ones by [Zhang 2001a, Propositions 7.1.1 and 6.4.5], and there is no extra term $h$. In fact (and with no restriction on $N$ ), these values also appear as the local components ${ }^{\mathbb{C}} \Phi_{v}^{\prime}$ at finite places of a form ${ }^{\mathbb{C}} \Phi^{\prime}$ of level $N$, which is a kernel of the Rankin-Selberg convolution for the central derivative $L^{\prime}\left(f_{E}, 1\right)$ of the complex $L$-function.

In general, [Zhang 2001b, Lemma 6.4.3] proves that ${ }^{42}$

$$
\frac{\Psi_{v}}{\ell_{F, v}\left(\pi_{v}\right)} \sim \frac{\mathbb{C}_{\Phi_{v}^{\prime \sharp}}}{\log N\left(\wp_{v}\right)}+{ }_{v} h
$$

where ${ }_{v} h$ is a modular form with zero projection onto the $f$-eigenspace (see the discussion at the very end of [Zhang 2001b]; the forms ${ }_{v} h$ come from intersections at bad places) and ${ }^{\mathbb{C}} \Phi^{\prime \sharp}$ is a form of level $N \Delta$ that is a kernel for the complex RankinSelberg convolution in level $N \Delta$ (in particular, it is modular and $\operatorname{Tr}_{\Delta}\left({ }^{\mathbb{C}} \Phi^{\prime \sharp}\right)=$ $\mathbb{C}_{\Phi^{\prime}}+h^{\prime}$, where $h^{\prime}$ is a modular form of level $N$ that is orthogonal to $f$ ). Applying the operator $\operatorname{Tr}_{\Delta}$ in (7.1.2), we recover the asserted formula.

7.2. Local heights at $\boldsymbol{p}, \boldsymbol{I}$. The following is the key result concerning the local heights at places dividing $p$. We assume that all primes $\wp$ of $F$ dividing $p$ are split in $E$.

Proposition 7.2.1. The arithmetic function $\mathscr{R}_{p}^{4} \Psi_{p}$ belongs to $\overline{\mathscr{Y}}_{N}^{\text {ord }} \subset \overline{\mathscr{Y}}$ ord , and

$$
l_{f_{\alpha}}\left(\mathscr{R}_{p}^{4} \Psi_{p}\right)=0 \text {. }
$$

${ }^{42}$ We are adapting the notation to our case. In [Zhang 2001b], the form $f$ is denoted by $\phi$ and the functions $v h$ are denoted by $v f$. 
The modularity assertion follows, as in [Nekovár 1995], by difference from the modularity of $\Psi$ (hence of $\mathscr{R}_{p}^{4} \Psi$ ) and the modularity of $\mathscr{R}_{p}^{4} \Psi_{\text {fin }}$ proved in Proposition 8.1.1 below.

The proof of the vanishing of the $f_{\alpha}$-component will be completed in Section 7.3 using the results of the rest of this subsection.

We start by fixing for the rest of this section a prime $\wp$ of $F$ dividing $p$. Fix an isomorphism $B_{\wp}=B \otimes_{F} F_{\wp} \cong M_{2}\left(F_{\wp}\right)$ identifying the local order $R_{\wp}$ with $M_{2}\left(\mathcal{O}_{F, \wp}\right)$ and the field $E \subset B$ with the diagonal matrices in $M_{2}\left(F_{\wp}\right)$. Let the divisors $\eta_{c}$ be as in (6.2.1), and denote

$$
H_{s}=H\left[\wp^{s}\right], \quad u_{s}=u\left(\wp^{s}\right) .
$$

Let $y_{s} \in X\left(H_{s}\right)$ be the CM point of conductor $\wp^{s}$ defined by

$$
y_{s}=\left[\left(x_{0}, \iota_{\wp}\left(\begin{array}{ll}
\pi^{s} & 1 \\
& 1
\end{array}\right)\right)\right],
$$

where $\iota_{\wp}: \boldsymbol{G L}_{2}\left(F_{\wp}\right) \rightarrow \widehat{B}^{\times}$is the natural inclusion and $\pi$ is a uniformiser at $\wp$.

Fix a place $w$ of $H$ above $\wp$; we still denote by $w$ the induced place on each $H_{s}$ and by $\mathfrak{p}$ the prime of $E$ lying below $w$. Since $\wp$ splits in $E$, by [Zhang 2001a, $\S 2.2]$, the CM points $y_{s}=\left[A_{s}\right]$ are ordinary, and their canonical submodules with respect to the reduction modulo $w$ are given by $A_{s}[\mathfrak{p}]$.

Proposition 7.2.2 (norm relations). Let $y_{s}$ be the system of CM points defined above.

(1) Let $m=m_{0} \wp^{n}$ be an ideal of $F$ with $m_{0}$ prime to $\wp N$. We have

$$
\left[T\left(m \wp^{r+2}\right)-2 T\left(m \wp^{r+1}\right)+T\left(m \wp^{r}\right)\right](\eta)=u_{n+r+2}^{-1} T\left(m_{0}\right) \operatorname{Tr}_{H_{n+r+2} / E}\left(y_{n+r+2}\right)
$$

as divisors on $X$.

(2) For all $s \geq 1$, we have

$$
T(\wp) y_{s}=\operatorname{Tr}_{H_{s+1, w} / H_{s, w}}\left(y_{s+1}\right)+y_{s-1} .
$$

(3) For all $s \geq 1$, we have

$$
\varphi\left(y_{s}\right)=y_{s-1},
$$

where $\varphi$ is the lift (6.1.1) of Frobenius with respect to the reduction modulo $w$.

Proof. By the multiplicativity of Hecke operators, it is enough to prove the statement of part (1) for $m_{0}=1$. A simple computation based on Proposition 6.3.1 shows that the left-hand side is equal to $\eta_{\wp^{n+r+2}}$. Since the Galois action of $\operatorname{Gal}\left(H_{n+r+2} / E\right)$ is simply transitive on $Y_{\wp^{n+r+2}}$, the right-hand side is also equal to $\eta_{\wp^{n+r+2}}$. 
For part (2), use the notation $[g]$ to denote $\left[\left(x_{0}, \iota_{\wp}(g)\right)\right]$. Then we have

$$
T(\wp) y_{s}=\sum_{j \in \mathscr{O}_{F, \wp} / \wp}\left[\left(\begin{array}{ll}
\pi^{s} & 1 \\
& 1
\end{array}\right)\left(\begin{array}{ll}
\pi & j \\
& 1
\end{array}\right)\right]+\left[\left(\begin{array}{ll}
\pi^{s} & 1 \\
& 1
\end{array}\right)\left(\begin{array}{ll}
1 & \\
& \pi
\end{array}\right)\right] .
$$

The last term is identified as $y_{s-1}$ after acting by the diagonal matrix $\pi^{-1}$ id (whose action is trivial on $X$ ). On the other hand, by local class field theory and the description of the Galois action on CM points of Section 6.2, we have

$$
\operatorname{Tr}_{H_{s+1, w} / H_{s, w}}\left(y_{s+1}\right)=\sum_{j \in \mathcal{O}_{F, \wp} / \wp}\left[\left(\begin{array}{ll}
1+j \pi^{s} & \\
& 1
\end{array}\right)\left(\begin{array}{ll}
\pi^{s+1} & 1 \\
& 1
\end{array}\right)\right],
$$

which is the same as the above sum in $j$.

For part (3), which in fact is not needed in what follows, we switch to the moduli description, ${ }^{43}$ so $y_{s}=\left[A_{s}\right]=\left[A_{D_{s}}\right]$ for an increasing sequence of admissible submodules $D_{s}$ of level $\wp^{s}$ (this follows from part (2), together with a variant for $s=0$ that we omit, and the moduli description of Hecke correspondences). Now $D_{1}$ is different from $\operatorname{can}(A)=A[\mathfrak{p}]$ since $\left[A_{A[\mathfrak{p}]}\right]$ has conductor 1 , and in fact each $D_{s}$ does not contain $A[\mathfrak{p}]$ since if it did then $\left[A_{s}\right]$ would be in the support of $T(\wp)^{s-1}\left[A_{A[p]}\right]$, which is easily seen ${ }^{44}$ to consist of CM points of conductor dividing $\wp^{s-1}$. It follows that the point $\varphi\left(\left[A_{s}\right]\right)=\left[A_{D_{s}+\operatorname{can}\left(A_{s}\right)}\right]=\left[A_{D_{s}+A[\mathfrak{p}]}\right]$ is in the support of $T(\wp)\left[A_{s}\right]$, but it is not one of the Galois conjugates of $y_{s+1}$ since as just seen it has lower conductor; by part (2), it must then be $y_{s-1}$.

Lemma 7.2.3. Let $w$ a place of $E$ dividing $\wp$, and let $h \in E_{w}(X)$ be a rational function whose reduction at $w$ is defined and nonzero. Let $\mu=\mu_{\wp}$ be the order of the ideal $\mathfrak{p}_{w}$ in the relative class group of $E / F$. Then the arithmetic functions

$$
\mathscr{R}_{\wp}^{2} \mathscr{R}_{\wp}^{(\mu)}\left\langle\widehat{\operatorname{div}}(h), T^{0}(m) \hat{\eta}\right\rangle_{w}^{\mathrm{Ar}}, \quad \mathscr{R}_{\wp}^{3}\langle\operatorname{div}(h), T(m) z\rangle_{w}
$$

belong to the kernel of the $\wp-p a r t i a l$ ordinary projection $e_{\wp}$.

Proof. We show more precisely that

$$
v\left(U_{\wp}^{s} \mathscr{R}_{\wp}^{3}\left\langle\widehat{\operatorname{div}}(h), T^{0}(m) \hat{\eta}\right\rangle_{w}^{\operatorname{Ar}}\right) \geq v\left(N \wp^{s}\right)-C
$$

for a uniform constant $C$, where $v$ is the $p$-adic valuation. We may assume $m$ prime to $\wp N \Delta$.

For the second expression, under our assumptions, $T\left(m \wp^{s}\right) \eta=T^{0}\left(m \wp^{s}\right) \eta+$ $r\left(m \wp^{s}\right) \eta-h \sigma_{1}\left(m \wp^{s}\right) \xi$, so the analogue of (7.2.1) holds with the same proof together

${ }^{43}$ As usual, after base change to a suitable quadratic extension $F^{\prime}$.

${ }^{44} \mathrm{By}$ the following observation: if $y$ is a CM point of conductor $c$, then the support of $T(m) y$ consists of $\mathrm{CM}$ points of conductors dividing $\mathrm{cm}$. 
with the observation that $\mathscr{R}_{\wp}^{2} r(m)=0$ and $v\left(\sigma_{1}\left(m \wp^{s}\right)\right)=v\left(N \wp^{s}\right)$ [Perrin-Riou 1987, Lemme 5.4].

As $\mathscr{R}_{\wp}^{2} r(m)=0$, Proposition 7.2.2(1) gives

$$
U_{\wp}^{s} \mathscr{R}_{\wp}^{2} \eta=u_{s+2}^{-1} \operatorname{Tr}_{H_{s+2} / E} y_{s+2}
$$

where $y_{s+2} \in Y_{\wp} s^{s+2}$; we make a compatible choice of $y_{s}$ such as the one described above Proposition 7.2.2.

For $s$ large enough, the divisor of $h$ is supported away from $y_{s}$ and its conjugates. Then by Proposition 5.4.1(3), we have

$$
\begin{aligned}
U_{\wp}^{s} \mathscr{R}_{\wp}^{2}\left\langle\widehat{\operatorname{div}}(h), T^{0}(m) \hat{\eta}\right\rangle_{w}^{\mathrm{Ar}} & =u_{s+2}^{-1} \ell_{w}\left(h\left(T^{0}(m) y_{s+2}\right)\right) \\
& =u_{s+2}^{-1} \sum_{w^{\prime} \mid w} \ell_{w}\left(N_{H_{s+2, w^{\prime}}} / E_{w} h\left(y_{s+2}\right)\right),
\end{aligned}
$$

where $w^{\prime}$ runs over the places of $H$ above $w$ (which are identified with the places of $H_{s+2}$ above $w$ since $H_{s+2} / H$ is totally ramified above $\left.\wp\right)$.

For any $w^{\prime} \mid w$, we have

$$
\begin{aligned}
\mathscr{R}_{\wp}^{(\mu)} \ell_{w}\left(N_{H_{s+2, w^{\prime}} / E_{w}} h\left(y_{s+2}\right)\right) \\
=\ell_{w} \circ N_{H_{w^{\prime}} / E_{w}}\left(N_{H_{s+2+\mu, w^{\prime}} / H_{w^{\prime}}} h\left(y_{s+2+\mu}\right) / N_{H_{s+2, w^{\prime}} / H_{w^{\prime}}} h\left(y_{s+2}\right)\right) .
\end{aligned}
$$

Suppose that (for $s$ large enough)

$$
\begin{aligned}
& \text { the } w^{\prime} \text {-adic valuation of } N_{H_{s, w^{\prime}} / H_{w^{\prime}}}\left(h\left(y_{s}\right)\right) \text { only } \\
& \text { depends on the residue class of } s(\bmod \mu) \text {. }
\end{aligned}
$$

Then each $w^{\prime}$-summand in the expression of interest is the product of $u_{s+2}^{-1}$ (which is eventually constant in $s$ ) and the $p$-adic logarithm of a unit that is a norm from an extension of $E_{w}$ whose ramification degree is a constant multiple of $N \wp^{s}$; hence, its $p$-adic valuation is also at least a constant multiple of the valuation of $N \wp^{s}$, which proves the lemma.

It remains to prove (7.2.2). We have

$$
w^{\prime}\left(N_{H_{s, w^{\prime}} / H_{w^{\prime}}}\left(h\left(y_{s}\right)\right)\right)=\left[H_{s, w^{\prime}}: H_{w^{\prime}}\right]\left(\underline{(h)}, \underline{y_{s}}\right),
$$

where the pairing in the right-hand side denotes the intersection multiplicity of the Zariski closures in the integral model. Now as in [Perrin-Riou 1987, Lemme 5.5], if $\pi_{s}$ denotes a uniformiser of $H_{s, w^{\prime}}$, we can show that we have

$$
\underline{y_{s}} \equiv \underline{y_{s-\mu}} \bmod \pi_{s}, \quad \underline{y_{s}} \not \equiv \underline{y_{s-\mu}} \bmod \pi_{s}^{2} .
$$

In fact, we first check that the two points have the same reduction. By [Zhang 2001b, Lemma 5.4.2], the set of points in the special fibre $\mathscr{X} \times_{\mathbb{O}_{F, \wp}} \overline{\boldsymbol{F}}_{\wp}$ having CM 
by $E$ (and thus being ordinary as $\wp$ splits in $E$ ) is identified with

$$
E^{\times} \backslash\left(N\left(F_{v}\right) \backslash \boldsymbol{G} \boldsymbol{L}_{2}\left(F_{\wp}\right)\right) \times \boldsymbol{B}^{\wp \infty \times} / \widehat{F}^{\times} \widehat{\boldsymbol{R}}^{\times}
$$

(where $N$ is the group of upper-triangular unipotent matrices) in such a way that the reduction map sends the $\mathrm{CM}$ point $\left[\left(x_{0}, g\right)\right] \in X(\mathbb{C})$ to the class of $g$. Then if $\sim$ denotes the equivalence relation in (7.2.5) and $t \in E^{\times}$is a generator of the ideal $\mathfrak{p}_{w}^{\mu} a$ for some ideal $a$ of $\hat{O}_{F}$ with adelic generator $\pi_{a}$, the reduction of $y_{s}$ is the class of

$$
\begin{aligned}
& \iota_{\wp}\left(\begin{array}{ll}
\pi^{s} & 1 \\
& 1
\end{array}\right) \sim \iota_{\wp}\left(\begin{array}{ll}
\pi^{s} & \\
& 1
\end{array}\right) \sim \iota_{\wp}\left(\left(\begin{array}{ll}
\pi^{\mu} & \\
& 1
\end{array}\right)\left(\begin{array}{ll}
\pi^{s-\mu} & \\
& 1
\end{array}\right)\right) \\
& \sim t \iota_{\wp}\left(\begin{array}{ll}
\pi^{s-\mu} & \\
& 1
\end{array}\right)\left(t^{\wp \infty}\right)^{-1} \pi_{a}^{-1} \sim \iota_{\wp}\left(\begin{array}{ll}
\pi^{s-\mu} & \\
& 1
\end{array}\right) \sim \iota_{\wp}\left(\begin{array}{ll}
\pi^{s-\mu} & \\
& 1
\end{array}\right),
\end{aligned}
$$

which is the same as the reduction of $y_{s-\mu}$.

We can then verify the congruence relation (7.2.4) on the completed local ring $\hat{\mathrm{O}}_{\mathscr{X} / W\left(\overline{\boldsymbol{F}}_{v}\right), \bar{y}}$ of the common reduction $\bar{y}$; here $W$ is the ring of integers in the completion of the maximal unramified extension of $F_{v}$. By [Carayol 1986, §5.5, Proposition], this is the universal deformation ring of the $p$-divisible module $\varphi_{\bar{y}} \frac{1}{1}$ (with the notation of Section 6.1). As the point $\bar{y}$ is ordinary, such module is isomorphic to the product $F_{\wp} / \mathscr{O}_{F, \wp} \times \Sigma_{1}$, where $\Sigma_{1}$ is the Lubin-Tate formal $\mathscr{O}_{F, \wp}$ module of height 1 . Now its lifting $\mathscr{G}_{s}^{1}=\mathscr{G}_{y_{s}}^{1}$ is defined precisely over the ring of integers of $H_{s, w^{\prime}}$, and so it is a quasicanonical lifting of level $s$ of its reduction $\mathscr{G}_{\bar{y}}$, in the sense of [Gross 1986]. Then by [Gross 1986, §6] (see also [Meusers 2007] for a detailed account), $\varphi_{s}^{1}$ is congruent to the canonical lifting modulo $\pi_{s}$ but not modulo $\pi_{s}^{2}$, whereas $\mathscr{G}_{s-\mu}^{1}$ is congruent to the canonical lifting modulo $\pi_{s-\mu}=\pi_{s}^{N \wp^{\mu}}$; this implies (7.2.4). Then for each irreducible component $\underline{a}$ in the support of $(h)$, the sequence $\left[H_{s, w^{\prime}}: H_{w^{\prime}}\right]\left(\underline{a}, \underline{y_{s}}\right)$ stabilises to either 0 or 1 so that the expression (7.2.3) is indeed eventually constant along the arithmetic progression.

Lemma 7.2.4. For each divisor $D \in \operatorname{Div}^{0}(X)\left(E_{v}\right)$ or $\widehat{D} \in \operatorname{Div}^{\operatorname{Ar}}(X)$, the element of $\bar{\varphi}$ ord given by

$$
m \mapsto \mathscr{R}_{\wp}^{2} \mathscr{R}_{\wp}^{(\mu)}\langle D, T(m) z\rangle_{w}, \quad m \mapsto \mathscr{R}_{\wp}^{2} \mathscr{R}_{\wp}^{(\mu)}\left\langle\widehat{D}, T^{0}(m) \hat{\eta}\right\rangle_{w}^{\mathrm{Ar}}
$$

is well-defined independently of the choice of $D$ in its class $[D]$ or $\widehat{D}$ in its class $[\widehat{D}]$, respectively; it will be denoted by

$$
\mathscr{R}_{\wp}^{2} \mathscr{R}_{\wp}^{(\mu)}\langle[D], T(m) z\rangle_{w}, \quad \mathscr{R}_{\wp}^{2} \mathscr{R}_{\wp}^{(\mu)}\left\langle[\widehat{D}], T^{0}(m) \hat{\eta}\right\rangle_{w}^{\mathrm{Ar}} .
$$

If $\widehat{D}=D$, then the two elements coincide as elements of $\overline{\mathcal{S}}^{\mathrm{ord}}$; moreover, for the arithmetic function $\Psi_{w} \in \overline{\mathscr{Y}}$ with $\Psi_{w}(m)=\left\langle\hat{\eta}, T^{0}(m) \hat{\eta}\right\rangle_{w}^{\mathrm{Ar}}$, we have

$$
\mathscr{R}_{\wp}^{2} \mathscr{R}_{\wp}^{(\mu)} \Psi_{w} \sim \mathscr{R}_{\wp}^{2} \mathscr{R}_{\wp}^{(\mu)}\langle[z], T(m) z\rangle_{w}
$$

in $\bar{\varphi}$ ord. 
Proof. The first part follows from Lemma 7.2.3. For the second part, we may argue as in the proof of Lemma 7.1.1: for example, in $\overline{\mathscr{Y}}$, we have

$$
\begin{aligned}
\mathscr{R}_{\wp}^{2} \mathscr{R}_{\wp}^{(\mu)}\left\langle\hat{\eta}, T^{0}(m) \hat{\eta}\right\rangle_{w}^{\mathrm{Ar}} & \sim \mathscr{R}_{\wp}^{2} \mathscr{R}_{\wp}^{(\mu)}\left\langle\hat{\eta}+\widehat{\operatorname{div}}(h), T^{0}(m) \hat{\eta}\right\rangle_{w}^{\mathrm{Ar}} \\
& \sim \mathscr{R}_{\wp}^{2} \mathscr{R}_{\wp}^{(\mu)}\langle\hat{z}+\widehat{\operatorname{div}}(h), T(m) \hat{z}\rangle_{w}^{\mathrm{Ar}} \\
& =\mathscr{R}_{\wp}^{2} \mathscr{R}_{\wp}^{(\mu)}\langle z+\operatorname{div}(h), T(m) z\rangle_{w} .
\end{aligned}
$$

7.3. Local heights at $\boldsymbol{p}, \boldsymbol{I I}$. Here we prove the vanishing statement for $p$-adic local symbols asserted in Proposition 7.2.1. In fact, we will show the equivalent statement

$$
l_{f_{\alpha}}\left(\mathscr{R}_{p}^{4} \mathscr{R}_{p}^{(\mu)} \Psi_{p}\right)=\prod_{\wp \mid p}\left(\alpha_{\wp}^{\mu_{\wp}}-1\right) l_{f_{\alpha}}\left(\mathscr{R}_{p}^{4} \Psi_{p}\right)=0,
$$

where the integers $\mu=\left(\mu_{\wp}\right)_{\wp} \mid p$ are as in Lemma 7.2.3.

Let $e_{f} \in \boldsymbol{T}_{N p} \otimes M_{f}$ be the maximal idempotent satisfying $T(m) \circ e_{f}=a(f, m) e_{f}$ for all $m$ prime to $N p ;{ }^{45}$ viewed as an endomorphism of $S_{N \prod_{\wp \mid p} \wp}$, it is the projector onto the subspace generated by $f$ and [ø] $f$ for all the primes $\wp$ of $F$ dividing $p$. With $z_{f}=e_{f}[z]$, we have by (7.2.6)

$$
e_{f} e \mathscr{R}_{p}^{4} \mathscr{R}_{p}^{(\mu)} \Psi_{p}=\mathscr{R}_{p}^{4} \mathscr{R}_{p}^{(\mu)}\left\langle z_{f}, T(m) z\right\rangle_{p}
$$

in $\overline{\mathscr{Y}}_{N}^{\text {ord }}$, where the left-hand side makes sense by the modularity part of Proposition 7.2.1 and the right-hand side makes sense by Lemma 7.2.4. We also denote, for $w$ a place of $E$ above the $F$-prime $\wp \mid p$, and $i \geq 2$,

$$
e_{f} e \mathscr{R}_{\wp}^{i} \mathscr{R}_{\wp}^{\left(\mu_{\wp}\right)} \Psi_{w}:=\mathscr{R}_{\wp}^{i} \mathscr{R}_{\wp}^{\left(\mu_{\wp}\right)}\left\langle z_{f}, T(m) z\right\rangle_{w},
$$

where the right-hand side makes sense as an element of $\overline{\mathscr{S}}^{\text {ord }}$ by Lemma 7.2.4. (As we have not shown that $\mathscr{R}_{\wp}^{3} \Psi_{w}$ is modular, the left-hand side is not otherwise defined.) Then by definition, we have

$$
e_{f} e \mathscr{R}_{p}^{4} \mathscr{R}_{p}^{(\mu)} \Psi_{p}=\sum_{w \mid p} e_{f} e \mathscr{R}_{p}^{4} \mathscr{R}_{p}^{(\mu)} \Psi_{w} .
$$

Now since $l_{f_{\alpha}}=l_{f_{\alpha}} \circ e_{f}=l_{f_{\alpha}} \circ e_{f} \circ e$, by (7.3.2), the desired result is implied by the following lemma for all $\wp \mid p$ :

Lemma 7.3.1. Suppose that $f$ is ordinary at $\wp$. For each place $w$ of $E$ above $\wp \mid p$, the element $e_{f} \mathscr{R}_{\wp}^{2} \mathscr{R}_{\wp}^{\left(\mu_{\wp)}\right)} \Psi_{w}$ is zero in $\overline{\mathcal{S}}$ ord .

Proof. The ordinarity assumption and Lemma 5.1.1 [Perrin-Riou 1987, Exemple 4.12] imply that $z_{f}$ is "almost" a universal norm in the totally ramified $\mathbb{Z}_{p^{-}}$ extension $E_{w, \infty}^{\ell}$ of $E_{w}$ : that is, after perhaps replacing $z_{f}$ by an integer multiple,

${ }^{45}$ Recall that $M_{f}$ is the number field generated by the Fourier coefficients $a(m, f)$. 
for each layer $E_{w, n}^{\ell}$, we have

$$
z_{f}=\operatorname{Tr}_{n}\left(z_{n}\right)
$$

for some $z_{n} \in e_{f} J(X)\left(E_{v, n}^{\ell}\right)$, where $\operatorname{Tr}_{n}=\operatorname{Tr}_{E_{w, n}^{\ell} / E_{w}}$. Then we have

$$
e_{f} \mathscr{R}_{\wp}^{2} \mathscr{R}_{\wp}^{\left(\mu_{\wp}\right)} \Psi_{w}(m)=\mathscr{R}_{\wp}^{2} \mathscr{R}_{\wp}^{\left(\mu_{\wp}\right)}\left\langle\operatorname{Tr}_{n}\left(z_{n}\right), T(m) z\right\rangle_{w}=\mathscr{R}_{\wp}^{2} \mathscr{R}_{\wp}^{\left(\mu_{\wp}\right)}\left\langle z_{n}, T(m) z\right\rangle_{w, n},
$$

where $\langle\cdot, \cdot\rangle_{w, n}$ is the local height pairing on $\operatorname{Div}^{0}(X)\left(E_{w, n}^{\ell}\right)$ associated with the $\operatorname{logarithm} \ell_{n, v}=\ell_{w} \circ N_{E_{w, n}^{\ell} / E_{w}}$. By Proposition 5.1.2(5)-(6), the right-hand side above has image in $c^{-1} \operatorname{Im}\left(\ell_{n}\right) \subset \mathbb{Z}_{p}$ for a uniform nonzero constant $c \in \mathbb{Z}_{p}$. As the extension $E_{w, n}^{\ell} / E_{w}$ has ramification degree $p^{n}$, we have for some nonzero $c^{\prime} \in \mathbb{Z}_{p}$

$$
e_{f} \mathscr{R}_{\wp}^{2} \mathscr{R}_{p}^{\left(\mu_{\wp}\right)} \Psi_{v}(m) \in c^{-1} \operatorname{Im}\left(\ell_{n}\right) \subset c^{\prime-1} p^{n} \mathbb{Z}_{p}
$$

for all $n$; therefore, $e_{f} \mathscr{R}_{\wp}^{2} \mathscr{R}_{p}^{\left(\mu_{\wp}\right)} \Psi_{w}=0$.

\section{Part III. Main theorem and consequences}

\section{Proof of the main theorem}

In this section, we prove Theorem B.

8.1. Basic case. First we prove the formula when $\Delta_{E / F}$ is totally odd and each prime $\wp$ of $F$ dividing $p$ splits in $E$.

Let $\Psi_{\mathscr{W}} \in \overline{\mathscr{Y}}_{N}$ denote the modular form with coefficients $\langle[z], T(m)[z]\rangle_{\mathscr{W}}$, where $\mathscr{W}=v \circ \mathfrak{N}$ and $\langle\cdot, \cdot\rangle_{\mathscr{W}}$ is the height pairing on $J(X)(E)$ associated with the $p$-adic $\operatorname{logarithm} \ell_{F} \circ \mathfrak{N}$, with

$$
\ell_{F}=\left.\frac{d}{d s}\right|_{s=0} \nu^{s}: F^{\times} \backslash F_{A^{\infty}}^{\times} \rightarrow \mathbb{Q}_{p} .
$$

Recall that $l_{f_{\alpha}}$ is a continuous functional so that it commutes with limits and

$$
L_{p, W}^{\prime}\left(f_{E}\right)(\mathbb{1})=l_{f_{\alpha}}\left(\left.\frac{d}{d s}\right|_{s=0} \Phi\left(\mathscr{W}^{s}\right)\right)=l_{f_{\alpha}}\left(\Phi_{\mathscr{W}}^{\prime}\right) .
$$

We compare the Fourier coefficients of $\Phi_{\mathscr{W}}^{\prime}$ and $\Psi_{\mathscr{W}}=\Psi_{\mathscr{W}, \text { fin }}+\Psi_{\mathscr{W}, p}$.

Proposition 8.1.1. Suppose that all of the prime ideals $\wp$ of $F$ dividing $p$ are principal. Then we have

$$
\left(\prod_{\wp \mid p} U_{\wp}^{4}-U_{\wp}^{2}\right) \Phi_{\mathscr{W}}^{\prime} \sim\left(\prod_{\wp \mid p}\left(U_{\wp}-1\right)^{4}\right) \Psi_{\mathscr{W}, \text { fin }}
$$

in the quotient space $\overline{\mathscr{Y}_{N}}$ ord $/ \operatorname{Ker}\left(l_{f_{\alpha}}\right)$. 
Proof. We prove that the identity holds in $\overline{\mathscr{S}} /\left(\mathscr{D}_{N}+\operatorname{Ker}\left(l_{f_{\alpha}}\right)\right)$, where $\operatorname{Ker}\left(l_{f_{\alpha}}\right)$ denotes the image in $\overline{\mathscr{S}}$ of classical modular form killed by $l_{f_{\alpha}}$. Then since the left-hand side belongs to $\overline{\mathscr{S}}_{N}^{p \text {-adic }}$, so does the right-hand side (and after further quotienting by $\operatorname{Ker}(e)$, we descend to $\left.\overline{\mathscr{Y}}_{N}^{\text {ord }} / \operatorname{Ker}\left(l_{f_{\alpha}}\right)\right)$.

The coefficients of $\Psi_{\text {fin }}=\Psi_{\mathscr{W}}$, fin are computed in Proposition 7.1.2. To lighten the notation, we write the explicit expression for $\Psi(m)=\sum_{v \text { nonsplit }} \Psi_{v}(m)$ as

$$
\Psi_{v}(m)=\sum_{\substack{n \in S_{v}([m]) \\ v_{\wp}(n m) \geq 0 \forall \wp \mid p}} c_{v}([n m]) r((1-n) m \Delta) r\left(n m \Delta / N \wp_{v}^{\varepsilon(v)}\right),
$$

where the value $c_{v}([\mathrm{~nm}])$ only depends on the prime-to- $p$ part of the fractional ideal $\mathrm{nm}$ and the set $S_{v}([\mathrm{~m}])$ only depends on $v$ and the prime-to- $p$ part of $m$; here $\varepsilon(v)=1$ if $v$ is inert and $\varepsilon(v)=0$ if $v$ is ramified.

The coefficients of $\Phi^{\prime}$ are computed in Proposition 4.5.3. They look "almost" the same in that, up to the modular form $h$ of Proposition 7.1.2, which is in $\operatorname{Ker}\left(l_{f_{\alpha}}\right)$, we have, when $m$ is divisible by every $\wp \mid p$,

$$
\Phi_{\mathscr{W}}^{\prime}(m)=\sum_{v \text { nonsplit }} \Psi_{v}^{[p]}(m)
$$

where for a product $P$ of some of the primes $\wp \mid p$ we denote

$$
\Psi_{v}^{[P]}(m)=\sum_{\substack{n \in S_{v}([m]) \\ v_{\wp}(n m) \geq 0 \forall \wp\left|p \\ v_{\wp}(n m)=0 \forall \wp\right| P}} c_{v}([n m]) r((1-n) m \Delta) r(n m \Delta / N) .
$$

Then it is enough to show that, for each $v \nmid p$, each $\wp \mid p$ and each $\wp \nmid P$ with $P$ as above, we have

$$
\left(U_{\wp}^{4}-U_{\wp}^{2}\right) \Psi_{v}^{[P \wp]}=\left(U_{\wp}-1\right)^{4} \Psi_{v}^{[P]} .
$$

For the sake of notation, we write the computation when $v$ is ramified in $E$ and

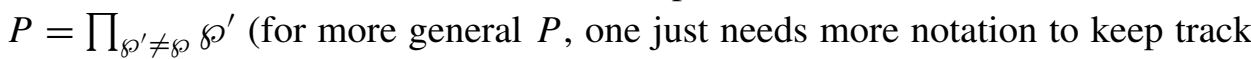
of $v_{\wp^{\prime}}(\mathrm{nm})$ for the primes $\left.\wp^{\prime} \neq \wp\right)$.

The right-hand side equals

$$
\begin{aligned}
& \sum_{i=0}^{4}(-1)^{i}\left(\begin{array}{l}
4 \\
i
\end{array}\right) \quad \sum_{n_{i} \in S_{0}([m])} \quad c_{v}\left(\left[n_{i} m\right]\right) r\left(\left(1-n_{i}\right) m \wp^{i} \Delta\right) r\left(n_{i} m \wp^{i} \Delta / N\right) . \\
& v_{\wp^{\prime}}\left(n_{i} m\right)=0 \forall \wp^{\prime} \neq \wp, \wp^{\prime} \mid p \\
& v_{\wp}\left(n_{i} m \wp^{i}\right) \geq 0
\end{aligned}
$$

From the relation $r\left(m_{0} \wp^{t}\right)=(t+1) r\left(m_{0}\right)$, valid for $\wp \nmid m_{0}$, we deduce the relations

$$
\begin{aligned}
& 2 r(m)=r(m \wp)+r\left(m \wp^{-1}\right), \\
& 2 r(m)=r\left(m \wp^{2}\right)+r\left(m \wp^{-2}\right) \text { if } \wp \mid m \text {, } \\
& 2 r(m)=r\left(m \wp^{2}\right)-r(m) \quad \text { if } \wp \nmid m \text {, }
\end{aligned}
$$


where we recall that $r(m)=0$ if $m$ is not an integral ideal. Then we can pick a totally positive generator in $F$ for the ideal $\wp$, which abusing notation we will still denote by $\wp$, and make the substitution $n_{i}=\wp^{t-i} n_{0}$ with $\wp^{t} \| n_{i} m \wp^{i}$ to write (8.1.1) as

$$
\sum_{t \geq 0} \sum_{\substack{n_{0} \in S_{v}(m) \\ \wp^{\prime}\left(n_{0} m\right)=0 \forall \wp \mid p}} c_{v}\left(\left[n_{0} m\right]\right) r\left(\left(n_{0} m\right)^{(\wp)}\right)(t+1) A_{t},
$$

where we recall that for an ideal $m$ we denote $m^{(\wp)}=m \wp^{-v_{\wp}(m)}$ and

$$
\begin{aligned}
A_{t}=r\left(m \Delta \wp^{4}\right. & \left.\left(1-n_{0} \wp^{t-4}\right)\right)[t+1-2 t+2(t-1)] \\
& +r\left(m \Delta \wp^{2}\left(1-n_{0} \wp^{t-2}\right)\right)\left[-2(t+2)+\left\{\begin{array}{cc}
4(t+1)-2 t & \text { if } t \geq 1, \\
3 & \text { if } t=0
\end{array}\right]\right. \\
& +r\left(m \Delta\left(1-n_{0} \wp^{t}\right)\right)[t+3-2(t+2)+t+1] .
\end{aligned}
$$

The three expressions in square brackets vanish when $t>0$ and yield, respectively, 1 , 1 and 0 when $t=0$. Substituting back $n_{4}=\wp^{t-4} n_{0}$ in the first line and $n_{2}=\wp^{t-2} n_{0}$ in the second line, we deduce that (8.1.1) equals

$$
\left(U_{\wp}^{4}-U_{\wp}^{2}\right) \Psi_{v}^{[P \wp]}
$$

as desired. ${ }^{46}$

Combining this proposition with Proposition 7.2.1, which says

$$
l_{f_{\alpha}}\left(\prod_{\wp \mid p}\left(U_{\wp}-1\right)^{4} \Psi_{W, p}\right)=0
$$

we find for $\mathscr{W}=v \circ \mathfrak{N}$

$$
\begin{aligned}
D_{F}^{2} \prod_{\wp}\left(\alpha_{\wp}^{4}-\alpha_{\wp}^{2}\right) L_{p, W}^{\prime}\left(f_{E}, \mathbb{1}\right) & =\prod_{\wp}\left(\alpha_{\wp}^{4}-\alpha_{\wp}^{2}\right)\left(1-\frac{1}{\alpha_{\wp}^{2}}\right)\left(1-\frac{N \wp}{\alpha_{\wp}^{2}}\right) l_{f_{\alpha}}\left(\Phi_{W}^{\prime}\right) \\
& =\prod_{\wp}\left(\alpha_{\wp}-1\right)^{4}\left(1-\frac{1}{\alpha_{\wp}^{2}}\right)\left(1-\frac{N \wp}{\alpha_{\wp}^{2}}\right) l_{f_{\alpha}}\left(\Psi_{W}\right) \\
& =\prod_{\wp}\left(\alpha_{\wp}-1\right)^{4}\left(1-\frac{1}{\alpha_{\wp}^{2}}\right)\left\langle z_{f}, z_{f}\right\rangle_{W} .
\end{aligned}
$$

Here, besides the definition of $L_{p}\left(f_{E}\right)$ (Definition 4.2.1), we have used various properties of the functional $l_{f_{\alpha}}$ from Lemma 1.6.1 and the observation that the projection onto the $f$-component of the modular form $\Psi_{W} \in S_{2}\left(K_{0}(N), \mathbb{Q}_{p}\right)$ is $\mathbb{1}_{f}\left(\Psi_{W}\right)=\left\langle z_{f}, z_{f}\right\rangle_{W}$.

This completes the proof of Theorem B when $\left(\Delta_{E / F}, 2\right)=1$ and all primes $\wp \mid p$ split in $E$. 
8.2. Reduction to the basic case. The general case, where $E$ is only assumed to satisfy $\left(\Delta_{E / F}, N p\right)=1$, can be reduced to the previous one under the assumption

$$
L_{p, \mathscr{W}}^{\prime}\left(f_{E}, \mathbb{1}\right) \neq 0
$$

by the following argument due to [Kobayashi 2013, proof of Theorem 5.9] using the complex Gross-Zagier formula (which is known with no restrictions on $\Delta$ ) and the factorisation $L_{p}\left(f_{E}, \chi \circ \mathfrak{N}\right) \sim L_{p}(f, \chi) L_{p}\left(f_{\varepsilon}, \chi\right)$.

By the factorisation, the orders of vanishing at the central point of the factors of $L_{p}\left(f_{E}, v^{s} \circ \mathfrak{N}\right.$ ) will be 1 (say for $L_{p}(f)$ ) and 0 (say for $L_{p}\left(f_{\varepsilon}\right)$ ). Then, by the first part of Theorem $\mathrm{C},{ }^{47}$ the orders of vanishing of $L(f, s)$ and $L\left(f_{\varepsilon}, s\right)$ at $s=1$ will also be 1 and 0 . Moreover, the Heegner point $z_{f, E^{\prime}}$ attached to $f$ and any $E^{\prime}$ also satisfying $L\left(f_{\varepsilon_{E^{\prime} / F}}, 1\right) \neq 0$ is nontorsion, and in fact, its trace $z_{f, F}=\operatorname{Tr}_{E^{\prime} / F}\left(z_{f, E^{\prime}}\right)$ is nontorsion and $z_{f, E^{\prime}}$ is up to torsion a multiple of $z_{f, F}$ in $J(X)\left(E^{\prime}\right) \otimes \overline{\mathbb{Q}}$. Therefore, by the complex and $p$-adic Gross-Zagier formulas for a suitable $E^{\prime}$ satisfying the assumptions of Section 8.1 and $L\left(f_{\varepsilon_{E^{\prime} / F}}, 1\right) \neq 0$, we have

$$
L_{p, \nu}^{\prime}(f, \mathbb{1})=\prod_{\wp \mid p}\left(1-\frac{1}{\alpha_{\wp}}\right)^{2} \frac{L^{\prime}(f, 1)}{\Omega_{f}^{+}\left\langle z_{f, F}, z_{f, F}\right\rangle}\left\langle z_{f, F}, z_{f, F}\right\rangle_{\nu},
$$

where $\langle\cdot, \cdot\rangle_{\nu}$ is the $p$-adic height pairing on $J(X)(F)$ attached to $v$ and $\langle\cdot, \cdot\rangle$ is the Néron-Tate height (the ratio appearing above belongs to $M_{f}^{\times}$by the Gross-Zagier formula). This allows us to conclude

$$
\begin{aligned}
L_{p, W}^{\prime}\left(f_{E}, \mathbb{1}\right) & =\frac{\Omega_{f}^{+} \Omega_{f_{\varepsilon}}^{+}}{D_{E}^{-1 / 2} \Omega_{f}} L_{p, v}^{\prime}(f, \mathbb{1}) L_{p}\left(f_{\varepsilon}, \mathbb{1}\right) \\
& =D_{E}^{1 / 2} \prod_{\wp \mid p}\left(1-\frac{1}{\alpha_{\wp}}\right)^{2}\left(1-\frac{\varepsilon(\wp)}{\alpha_{\wp}}\right)^{2} \frac{L^{\prime}(f, 1) L\left(f_{\varepsilon}, 1\right)}{\Omega_{f}\left\langle z_{f, F}, z_{f, F}\right\rangle}\left\langle z_{f, F}, z_{f, F}\right\rangle_{v} \\
& =D_{F}^{-2} \prod_{\wp \mid p}\left(1-\frac{1}{\alpha_{\wp}}\right)^{2}\left(1-\frac{\varepsilon(\wp)}{\alpha_{\wp}}\right)^{2} \frac{\left\langle z_{f, E}, z_{f, E}\right\rangle}{\left\langle z_{f, F}, z_{f, F}\right\rangle}\left\langle z_{f, F}, z_{f, F}\right\rangle_{v} \\
& =D_{F}^{-2} \prod_{\wp \mid p}\left(1-\frac{1}{\alpha_{\wp}}\right)^{2}\left(1-\frac{\varepsilon(\wp)}{\alpha_{\wp}}\right)^{2}\left\langle z_{f, E}, z_{f, E}\right\rangle W .
\end{aligned}
$$

Remark 8.2.1. It is natural to conjecture that when $L_{p, W}^{\prime}\left(f_{E}, \mathbb{1}\right)=0$ we should have $\left\langle z_{f}, z_{f}\right\rangle_{w}=0$. However, in this case, the above argument fails because, without knowledge of the nontriviality of the $p$-adic height pairing, the vanishing of $L_{p}\left(f_{E}, W^{s}\right)$ to order $\geq 2$ does not imply a similar high-order vanishing for $L\left(f_{E}, s\right)$.

${ }^{47}$ Which can be proved by using the $p$-adic Gross-Zagier formula attached to a field $E^{\prime}$ satisfying the assumptions of Section 8.1. 


\section{Periods and the Birch and Swinnerton-Dyer conjecture}

As seen in the introduction, the application of our result to the Birch and SwinnertonDyer formula rests on a conjectural relation among the periods of $f$ and the associated abelian variety $A$. Here we would like to briefly elaborate on this conjecture and its arithmetic consequences. (This section contains no new results or conjectures and is a very brief survey of work of Shimura and [Yoshida 1994].) We retain the notation of the introduction and set $M=M_{f}$ and $\operatorname{dim} A=[M: \mathbb{Q}]=d$.

9.1. Real periods. The conjecture on periods stated in the introduction can be refined to a conjecture on rationality rather than algebraicity. First we need to define the automorphic periods $\Omega_{f^{\sigma}}^{+}$for $\sigma \in \operatorname{Hom}(M, \mathbb{C})$; they are naturally defined as elements of $\mathbb{C}^{\times} / M^{\times}$(see [Raghuram and Tanabe 2011] for a modern exposition): one can choose them "covariantly" in the sense of [Yoshida 1994] in order to have $\prod_{\sigma} \Omega_{f^{\sigma}}^{+}$defined up to $\mathbb{Q}^{\times}$or define directly the product as follows. Let $\mathscr{H}_{N}=Z(\boldsymbol{A}) \backslash \boldsymbol{G} \boldsymbol{L}_{2}(\boldsymbol{A}) / K_{0}(N) K_{\infty}$ be the open Hilbert modular variety of level $N$. Then the perfect pairing of $\mathbb{Q}$-vector spaces

$$
H_{g}\left(\mathscr{H}_{N}, \mathbb{Q}\right)^{+} \times S_{2}\left(K_{0}(N), \mathbb{Q}\right) \rightarrow \mathbb{C}
$$

(where + denotes the intersection of the +1 -eigenspaces for the complex conjugations) decomposes under the diagonal action of $\boldsymbol{T}_{N}$ into $\mathbb{Q}$-rational blocks parametrised by the Galois-conjugacy classes of eigenforms. Then

$$
\prod_{\sigma} \Omega_{f^{\sigma}}^{+} \in \mathbb{C}^{\times} / \mathbb{Q}^{\times}
$$

is $(2 \pi i)^{d g}$ times the discriminant of the pairing on the rational block corresponding to $\left\{f^{\sigma}\right\}_{\sigma}$. (The individual $\Omega_{f^{\sigma}}^{+} \in \mathbb{C}^{\times} / M^{\times}$are defined as the discriminants of (9.1.1) on $\overline{\mathbb{Q}}$-rational $\boldsymbol{T}_{N}$-eigenblocks. One can similarly define periods $\Omega_{f^{\sigma}}^{-}$by paring with $H_{g}\left(\mathscr{H}_{N}, \mathbb{Q}\right)^{-}$, the -1 -eigenspace for the complex conjugations.)

Conjecture 9.1.1. We have

$$
\Omega_{A} \sim \prod_{\sigma} \Omega_{f^{\sigma}}^{+}
$$

in $\mathbb{C}^{\times} / \mathbb{Q}^{\times}$.

The conjecture is originally due [Shimura 1988, especially §11] and was refined by [Yoshida 1994]. When $A$ has complex multiplication, it has been proved by [Blasius 1986]. It is also known when $F=\mathbb{Q}$; before discussing that, let us translate it into a language closer to conjectures of Shimura. 
For each $\tau: F \rightarrow \mathbb{R}$, let $f_{B(\tau)}$ be the Jacquet-Langlands transfer of $f$ to a rational $^{48}$ form on the quaternion algebra $B(\tau) / F$ defined in the introduction (recall that $B(\tau)$ is ramified at all infinite places except $\tau$ ), and let $X$ be our Shimura curve. Then $A$ is (up to isogeny) a quotient $\phi$ of $J(X)$, and for each embedding $\tau$, we can write

$$
\phi^{*} \omega_{A}=c_{\tau} \bigwedge_{\sigma} 2 \pi i f_{B(\tau)}^{\sigma}(z) d z
$$

as forms in $H^{0}\left(J(X)\left(\mathbb{C}_{\tau}\right), \Omega^{d}\right.$ ) for some $c_{\tau} \in F^{\times}$(since both are generators of a rank-1 $F$-vector space); here $z$ denotes the coordinate on the upper half-plane uniformising $X$. Then we have

$$
\int_{A\left(\mathbb{R}_{\tau}\right)}\left|\omega_{A}\right|_{\tau} \sim \prod_{\sigma} \Omega_{f_{B(\tau)}^{\sigma}}^{+} \text {in } \mathbb{C}^{\times} / F^{\times},
$$

where $\Omega_{f_{(\tau)}^{\sigma}}^{+}$is $2 \pi i$ times the discriminant of the $f_{B(\tau)}^{\sigma}$-part of the analogue of the pairing (9.1.1) on $X\left(\mathbb{C}_{\tau}\right)$. When choices are made covariantly in $\tau$, we then get $\Omega_{A} \sim \prod_{\sigma, \tau} \Omega_{f_{B(\tau)}^{\sigma}}^{+}$in $\mathbb{C}^{\times} / \mathbb{Q}^{\times}$.

Our conjecture, decomposed into its $\sigma$-constituents, can then be rewritten as

$$
\Omega_{f}^{+} \sim \prod_{\tau} \Omega_{f_{B(\tau)}}^{+} \text {in } \mathbb{C}^{\times} /(M F)^{\times} .
$$

In this form, this is a stronger version of Shimura's conjecture [1983] on the factorisation of periods of Hilbert modular forms up to algebraic factors in terms of $P$-invariants. The reader is referred to [Yoshida 1994] for a discussion of this point.

Notice that (9.1.2) is nontrivial even when $F=\mathbb{Q}$ : it asserts that the periods of the transfers of $f$ to any indefinite quaternion algebra have the same transcendental (or irrational) parts. However, in this case, the conjecture is known by [Shimura 1981] (for the algebraicity) and [Prasanna 2009] (for the rationality).

For general $F$, Shimura's conjecture on $P$-invariants is largely proved by [Yoshida 1995] under an assumption of nonvanishing of certain $L$-values.

Remark 9.1.2. It is clear that our conjecture implies that the Birch and SwinnertonDyer conjectural formula is true up to a nonzero rational factor when $A$ has analytic $M$-rank 0. By the complex and $p$-adic Gross-Zagier formulas, the conjecture for $f$ also implies the complex and $p$-adic Birch and Swinnerton-Dyer formulas, respectively, up to a rational factor when $A$ has ( $p$-adic) analytic $M$-rank 1 .

9.2. Quadratic periods. We can formulate a conjecture analogous to Conjecture 9.1.1 for the periods of the base-changed abelian variety $A_{E}=A \times_{\operatorname{Spec} F} \operatorname{Spec} E$.

${ }^{48}$ For consistency with the case in which $B(\tau)=\boldsymbol{G} \boldsymbol{L}_{2}(\mathbb{Q})$ and "rational" means "rational $q$ expansion coefficients", here $f_{B(\tau)}$ is considered $F$-rational for the structure $H^{0}\left(X / F, \Omega_{X / F}\right) \otimes$ $(2 \pi i)^{-1} \mathbb{Q} \subset H^{0}\left(X / F, \Omega_{X / F}\right) \otimes_{F, \tau} \mathbb{C}$ (where $X$ is the Shimura curve defined in the introduction). 
Conjecture 9.2.1. We have

$$
\Omega_{A_{E}} \sim \prod_{\sigma} \Omega_{f^{\sigma}}
$$

in $\mathbb{C}^{\times} / \mathbb{Q}^{\times}$.

Here the period of $A_{E}$ is

$$
\Omega_{A_{E}}=\prod_{\tau: E \rightarrow \mathbb{C}} \int_{A\left(\mathbb{C}_{\tau}\right)}\left|\omega_{A_{E}}\right|_{\tau},
$$

where for a differential form $\omega=h(z) d z_{1} \wedge \cdots \wedge d z_{k}$ we have $|\omega|_{\tau}=|h(z)|_{\tau}^{2} d z_{1} \wedge$ $d \bar{z}_{1} \wedge \cdots \wedge d z_{k} \wedge d \bar{z}_{k}$.

As above, this conjecture can be "decomposed" into

$$
\Omega_{f} \sim \prod_{\tau} \Omega_{f_{B(\tau)}} \text { in } \mathbb{C}^{\times} /(M F)^{\times},
$$

where $\Omega_{f_{B(\tau)}}$ is $\pi^{2}$ times the Pertersson inner product of $f_{B(\tau)}$. This is essentially Shimura's conjecture on $Q$-invariants [1983]. Up to algebraicity, it has been proved by [Harris 1993] under a local condition (a new proof of the same result should appear in forthcoming work of Ichino and Prasanna, yielding rationality and removing the local assumption). As $\Omega_{f}=\Omega_{f}^{+} \Omega_{f}^{-}$, ${ }^{49}$ the factorisation (9.2.1) is implied by (9.1.2) and its analogue for $\Omega_{f}^{-}$; thus, Harris's result can be seen as evidence for the conjecture on real periods.

We take the opportunity to record an immediate consequence of the conjecture on quadratic periods and the Gross-Zagier formulas.

Theorem 9.2.2. If $A_{E}$ has complex or $p$-adic analytic $M$-rank $\leq 1$, then the complex or p-adic Birch and Swinnerton-Dyer formula, respectively, for $A_{E}$ is true up to a nonzero algebraic factor.

\section{List of symbols}

Throughout this text, we use the following notation and assumptions, unless otherwise noted:

- $F$ is a totally real field of degree $g$.

- $N_{F}$ is the monoid of nonzero ideals of $\mathscr{O}_{F}$.

- $|\cdot|_{v}$ is the standard absolute value on $F_{v}$.

- $\boldsymbol{A}=\boldsymbol{A}_{F}$ is the adele ring of $F$; if $*$ is a place or a set of places or an ideal of $F$, the component at $*$ or away from $*$ of an adelic object $x$ is denoted $x_{*}$ or $x^{*}$, respectively. For example if $\phi=\prod_{v} \phi_{v}$ is a Hecke character and $\delta$ is an

${ }^{49}$ See, e.g., [Shimura 1978, Theorem 4.3 (II)], where the assumption on the weight can now be removed thanks to the work of Rohrlich. 
ideal of $O_{F}$, we write $\phi_{\delta}(y)=\prod_{v \mid \delta} \phi_{v}\left(y_{v}\right)$ and $|y|_{\delta}=\prod_{v \mid \delta}|y|_{v}$. We also use the notation

$$
|m|_{v}=\left|\pi_{m}\right|_{v}, \quad|m|_{\delta}=\left|\pi_{m}\right|_{\delta}, \quad \phi_{v}(m)=\phi_{v}\left(\pi_{m}\right), \quad \phi_{\delta}(m)=\phi_{\delta}\left(\pi_{m}\right)
$$

if $m$ is an ideal of $\hat{O}_{F}$ and $\phi$ is unramified at $\delta$ (here $\pi_{m}$ satisfies $\pi_{m} \hat{O}_{F}=m$ ).

- $>$ denotes the partial order on $\boldsymbol{A}_{F}$ given by $x>0$ if and only if $x_{\infty}$ is totally positive.

- $R_{\boldsymbol{A}}=R \otimes_{F} \boldsymbol{A}$ if $R$ is an $F$-algebra.

- $\mathrm{Nm}$ is the absolute norm of an ideal $m$ in a number field (the index of $m$ in the ring of integers: it is a positive natural number).

- $d_{F}$ is the different of $F$.

- $\pi_{N}$, for $N$ an ideal of $O_{F}$, is the idele with components $\pi_{v}^{v(N)}$ for $v \nmid \infty$ and 1 for $v \mid \infty$.

- $D_{F}=N d_{F}$ is the discriminant of $F$.

- $m^{\times}=\left\{a \in F_{\boldsymbol{A}}^{\times} \mid a \mathfrak{O}_{F}=m\right\}$ if $m$ is any nonzero fractional ideal of $F$ (this notation will be used with $m=d_{F}^{-1}$ ).

- $E$ is a quadratic CM (that is, totally imaginary) extension of $F$.

- $\mathfrak{D}=\mathfrak{D}_{E / F}$ is the different of $E / F$.

- $\mathfrak{N}=N_{E / F}$ is the relative norm on $E$ or any $E$-algebra.

- $\Delta=\Delta_{E / F}=\mathfrak{N}(\mathfrak{D})$ is the relative discriminant of $E / F$, and we assume

$$
\left(\Delta_{E / F}, D_{F} N p\right)=1
$$

in Sections 2.5, 4.5 and part of 3.2, we further assume that

$$
(\Delta, 2)=1
$$

and in Sections 7.2, 7.3 and 8.1 that

$$
(\Delta, 2)=1 \text { and all primes } \wp \text { dividing } p \text { are split in } E \text {. }
$$

- $D_{E}=N(\Delta)$ is the absolute discriminant of $E$.

- $U_{F}(N)$ is the subgroup of $\hat{O}_{F}^{\times}=\prod_{v} \mathcal{O}_{F, v}^{\times} \subset F_{A^{\infty}}^{\times}$consisting of elements $x \equiv$ $1 \bmod N \hat{O}_{F}$, if $N$ is any ideal of $\hat{O}_{F}$.

- $\boldsymbol{e}_{v}(x)=\exp \left(-2 \pi i\left\{\operatorname{Tr}_{F_{v} / \mathbb{Q}_{p}}(x)\right\}_{p}\right)$ for $v \mid p<\infty$ and $\{y\}_{p}$ the $p$-fractional part of $y \in \mathbb{Q}_{p}$ is the standard additive character of $F_{v}$, with conductor $d_{F, v}^{-1}$; for $v \mid \infty, \boldsymbol{e}_{v}(x)=\exp \left(2 \pi i \operatorname{Tr}_{F_{v} / \mathbb{R}}(x)\right)$.

- $\boldsymbol{e}(x)=\prod_{v} \boldsymbol{e}_{v}\left(x_{v}\right)$ is the standard additive character of $\boldsymbol{A}_{F}$.

- $\mathbb{1}_{Y}$ is the characteristic function of the set $Y$.

- If $\varphi$ is any logical proposition, we define $\mathbb{1}[\varphi]$ to be 1 when $\varphi$ is true and 0 when $\varphi$ is false - e.g., $\mathbb{1}[x \in Y]=\mathbb{1}_{Y}(x)$. 


\section{Acknowledgements}

The present paper grew out of my Columbia thesis. I am grateful to my advisor Professor Shou-Wu Zhang for suggesting this area of research and for his support and encouragement and to Amnon Besser, Shinichi Kobayashi, Luis Garcia Martinez, David Loeffler, Yifeng Liu, Giovanni Rosso, Eric Urban, Jeanine Van Order and Shou-Wu Zhang for useful conversations or correspondence.

This work builds upon the works of Perrin-Riou [1987] and Zhang [2001a; 2001b; 2004] — and, of course, Gross and Zagier [1986]. My debt to their ideas cannot be overstated and will be obvious to the reader.

Some revisions to the manuscript were done while the author was a postdoctoral fellow at MSRI funded under NSF grant 0932078000.

\section{References}

[Andreatta and Goren 2005] F. Andreatta and E. Z. Goren, Hilbert modular forms: $\bmod p$ and $p$-adic aspects, Mem. Amer. Math. Soc. 819, Amer. Math. Soc., Providence, RI, 2005. MR 2006f:11049 Zbl 1071.11023

[Bertrand 1984] D. Bertrand, "Propriétés arithmétiques de fonctions thêta à plusieurs variables", pp. 17-22 in Number theory (Noordwijkerhout, Netherlands, 1983), edited by H. Jager, Lecture Notes Math. 1068, Springer, Berlin, 1984. MR 756080 Zbl 0546.14029

[Besser 2004] A. Besser, “The p-adic height pairings of Coleman-Gross and of Nekovář”, pp. 13-25 in Number theory (Montreal, 2002), edited by H. Kisilevsky and E. Z. Goren, CRM Proc. Lecture Notes 36, Amer. Math. Soc., Providence, RI, 2004. MR 2005f:11130 Zbl 1153.11316

[Besser 2005] A. Besser, "p-adic Arakelov theory", J. Number Theory 111:2 (2005), 318-371. MR 2006j:14029 Zbl 1079.14033

[Blasius 1986] D. Blasius, "On the critical values of Hecke L-series", Ann. Math. (2) 124:1 (1986), 23-63. MR 88i:11035 Zbl 0608.10029

[Bump 1997] D. Bump, Automorphic forms and representations, Cambridge Stud. Adv. Math. 55, Cambridge Univ., 1997. MR 97k:11080 Zbl 0868.11022

[Bump et al. 1990] D. Bump, S. Friedberg, and J. Hoffstein, "Nonvanishing theorems for $L$-functions of modular forms and their derivatives", Invent. Math. 102:3 (1990), 543-618. MR 92a:11058 Zbl 0721.11023

[Bushnell and Henniart 2006] C. J. Bushnell and G. Henniart, The local Langlands conjecture for GL(2), Grundlehren der math. Wissenschaften 335, Springer, Berlin, 2006. MR 2007m:22013 Zbl 1100.11041

[Carayol 1986] H. Carayol, "Sur la mauvaise réduction des courbes de Shimura", Compositio Math. 59:2 (1986), 151-230. MR 88a:11058 Zbl 0607.14021

[Casselman 1973] W. Casselman, "On some results of Atkin and Lehner", Math. Ann. 201:4 (1973), 301-314. MR 49 \#2558 Zbl 0239.10015

[Coleman and Gross 1989] R. F. Coleman and B. H. Gross, “p-adic heights on curves”, pp. 73-81 in Algebraic number theory (Berkeley, CA, 1987), edited by J. Coates et al., Adv. Stud. Pure Math. 17, Academic, Boston, 1989. MR 92d:11057 Zbl 0758.14009

[Deligne and Ribet 1980] P. Deligne and K. A. Ribet, "Values of abelian $L$-functions at negative integers over totally real fields", Invent. Math. 59:3 (1980), 227-286. MR 81m:12019 Zbl 0434.12009 
[Dimitrov 2013] M. Dimitrov, "Automorphic symbols, $p$-adic $L$-functions and ordinary cohomology of Hilbert modular varieties", Amer. J. Math. 135:4 (2013), 1117-1155. MR 3086071 Zbl 06203659

[Disegni 2015] D. Disegni, "The p-adic Gross-Zagier formula on Shimura curves", preprint, 2015, Available at http://www.math.mcgill.ca/disegni/papers/pyzz-pst.pdf.

[Faltings 1983] G. Faltings, "Endlichkeitssätze für abelsche Varietäten über Zahlkörpern”, Invent. Math. 73:3 (1983), 349-366. MR 85g:11026a Zbl 0588.14026

[Fontaine 1982] J.-M. Fontaine, "Sur certains types de représentations $p$-adiques du groupe de Galois d'un corps local; construction d'un anneau de Barsotti-Tate", Ann. Math. (2) 115:3 (1982), 529-577. MR 84d:14010 Zbl 0544.14016

[Freitas et al. 2015] N. Freitas, B. V. Le Hung, and S. Siksek, "Elliptic curves over real quadratic fields are modular", Invent. Math. 201:1 (2015), 159-206. MR 3359051 Zbl 06468724

[Gross 1986] B. H. Gross, "On canonical and quasi-canonical liftings", Invent. Math. 84:2 (1986), 321-326. MR 87g:14051 Zbl 0597.14044

[Gross and Zagier 1986] B. H. Gross and D. B. Zagier, "Heegner points and derivatives of $L$-series", Invent. Math. 84:2 (1986), 225-320. MR 87j:11057 Zbl 0608.14019

[Harris 1993] M. Harris, " $L$-functions of $2 \times 2$ unitary groups and factorization of periods of Hilbert modular forms”, J. Amer. Math. Soc. 6:3 (1993), 637-719. MR 93m:11043 Zbl 0779.11023

[Hida 1985] H. Hida, "A $p$-adic measure attached to the zeta functions associated with two elliptic modular forms, I”, Invent. Math. 79:1 (1985), 159-195. MR 86m:11097 Zbl 0573.10020

[Hida 1991] H. Hida, "On $p$-adic $L$-functions of GL(2) $\times$ GL(2) over totally real fields", Ann. Inst. Fourier (Grenoble) 41:2 (1991), 311-391. MR 93b:11052 Zbl 0725.11025

[Hida and Tilouine 1993] H. Hida and J. Tilouine, "Anti-cyclotomic Katz p-adic $L$-functions and congruence modules”, Ann. Sci. École Norm. Sup. (4) 26:2 (1993), 189-259. MR 93m:11044 Zbl 0778.11061

[Howard 2004] B. Howard, "Iwasawa theory of Heegner points on abelian varieties of GL 2 type", Duke Math. J. 124:1 (2004), 1-45. MR 2005f:11117 Zbl 1068.11071

[Hsieh 2014] M.-L. Hsieh, "Eisenstein congruence on unitary groups and Iwasawa main conjectures for CM fields”, J. Amer. Math. Soc. 27:3 (2014), 753-862. MR 3194494 Zbl 06346502

[Iovita 2000] A. Iovita, "Formal sections and de Rham cohomology of semistable abelian varieties", Israel J. Math. 120:B (2000), 429-447. MR 2002g:14026 Zbl 1045.14503

[Jacquet 1972] H. Jacquet, Automorphic forms on GL(2), part II, Lecture Notes Math. 278, Springer, Berlin, 1972. MR 58 \#27778 Zbl 0243.12005

[Kobayashi 2013] S. Kobayashi, "The $p$-adic Gross-Zagier formula for elliptic curves at supersingular primes”, Invent. Math. 191:3 (2013), 527-629. MR 3020170 Zbl 1300.11053

[Kobayashi 2014] S. Kobayashi, "The $p$-adic height pairing on abelian varieties at non-ordinary primes", pp. 265-290 in Iwasawa theory 2012: state of the art and recent advances (Heidelberg, 2012), edited by T. Bouganis and O. Venjakob, Contrib. Math. Comput. Sci. 7, Springer, Berlin, 2014. Zbl 06455256

[Kolyvagin 1988] V. A. Kolyvagin, "Finiteness of $E(\mathbf{Q})$ and $\amalg(E, \mathbf{Q})$ for a subclass of Weil curves", Izv. Akad. Nauk SSSR Ser. Mat. 52:3 (1988), 522-540. In Russian; translated in Math. USSR Izv. 32:3 (1989), 523-541. MR 89m:11056 Zbl 0662.14017

[Kolyvagin 1991] V. A. Kolyvagin, "On the structure of Shafarevich-Tate groups", pp. 94-121 in Algebraic geometry (Chicago, 1989), edited by S. Bloch et al., Lecture Notes Math. 1479, Springer, Berlin, 1991. MR 94b:11055 Zbl 0753.14025 
[Kolyvagin and Logachëv 1991] V. A. Kolyvagin and D. Y. Logachëv, "Finiteness of Ш over totally real fields", Izv. Akad. Nauk SSSR Ser. Mat. 55:4 (1991), 851-876. In Russian; translated in Math. USSR Izv. 39:1 (1992), 829-853. MR 93d:11063 Zbl 0791.14019

[Le Hung 2014] B. V. Le Hung, Modularity of some elliptic curves over totally real fields, Ph.D. thesis, Harvard University, 2014, Available at http://search.proquest.com/docview/1557761503. MR 3251352

[Manin 1976] Yu. I. Manin, "Non-Archimedean integration and $p$-adic Jacquet-Langlands $L$ functions”, Uspekhi Mat. Nauk 31:1 (1976), 5-54. In Russian; translated in Russ. Math. Surv. 31:1 (1976), 5-57. MR 54 \#5194 Zbl 0348.12016

[Meusers 2007] V. Meusers, "Canonical and quasi-canonical liftings in the split case", pp. 87-98 in Argos seminar on intersections of modular correspondences (Bonn, 2003-2004), Astérisque 312, Société Math. France, Paris, 2007. MR 2008g:11101 Zbl 1223.14051

[Nekovář 1993] J. Nekovář, "On p-adic height pairings", pp. 127-202 in Séminaire de Théorie des Nombres (Paris, 1990-1991), edited by S. David, Progr. Math. 108, Birkhäuser, Boston, 1993. MR 95j:11050 Zbl 0859.11038

[Nekovář 1995] J. Nekovář, "On the p-adic height of Heegner cycles", Math. Ann. 302:4 (1995), 609-686. MR 96f:11073 Zbl 0841.11025

[Panchishkin 1988] A. A. Panchishkin, "Convolutions of Hilbert modular forms and their nonArchimedean analogues", Mat. Sb. (N.S.) 136:4 (1988), 574-587. In Russian; translated in Math. USSR Sb. 64:2 (1989), 571-584. MR 89k:11033 Zbl 0656.10021

[Perrin-Riou 1987] B. Perrin-Riou, "Points de Heegner et dérivées de fonctions $L$ p-adiques", Invent. Math. 89:3 (1987), 455-510. MR 89d:11034 Zbl 0645.14010

[Perrin-Riou 1988] B. Perrin-Riou, "Fonctions $L$ p-adiques associées à une forme modulaire et à un corps quadratique imaginaire", J. London Math. Soc. (2) 38:1 (1988), 1-32. MR 89m:11043 Zbl 0656.10019

[Prasanna 2009] K. Prasanna, "Arithmetic properties of the Shimura-Shintani-Waldspurger correspondence”, Invent. Math. 176:3 (2009), 521-600. MR 2011d:11102 Zbl 1213.11102

[Raghuram and Tanabe 2011] A. Raghuram and N. Tanabe, "Notes on the arithmetic of Hilbert modular forms", J. Ramanujan Math. Soc. 26:3 (2011), 261-319. MR 2012m:11060 Zbl 1272.11069

[Schneider 1985] P. Schneider, "p-adic height pairings, II", Invent. Math. 79:2 (1985), 329-374. MR 86j:11063 Zbl 0571.14021

[Serre 1978] J.-P. Serre, "Sur le résidu de la fonction zêta $p$-adique d'un corps de nombres", $C$. $R$. Acad. Sci. Paris Sér. A 287:4 (1978), A183-A188. MR 58 \#22024 Zbl 0393.12026

[Shimura 1978] G. Shimura, "The special values of the zeta functions associated with Hilbert modular forms", Duke Math. J. 45:3 (1978), 637-679. MR 80a:10043 Zbl 0394.10015

[Shimura 1981] G. Shimura, "The periods of certain automorphic forms of arithmetic type", J. Fac. Sci. Univ. Tokyo Sect. IA Math. 28:3 (1981), 605-632. MR 84f:10040 Zbl 0499.10027

[Shimura 1983] G. Shimura, "Algebraic relations between critical values of zeta functions and inner products”, Amer. J. Math. 105:1 (1983), 253-285. MR 84j:10038 Zbl 0518.10032

[Shimura 1988] G. Shimura, "On the critical values of certain Dirichlet series and the periods of automorphic forms”, Invent. Math. 94:2 (1988), 245-305. MR 90e:11069 Zbl 0656.10018

[Shnidman 2014] A. Shnidman, " $p$-adic heights of generalized Heegner cycles", preprint, 2014. arXiv $1407.0785 \mathrm{v} 2$

[Skinner and Urban 2014] C. Skinner and E. Urban, "The Iwasawa main conjectures for GL 2", Invent. Math. 195:1 (2014), 1-277. MR 3148103 Zbl 1301.11074 
[Urban 2014] E. Urban, "Nearly overconvergent modular forms", pp. 401-441 in Iwasawa theory 2012: state of the art and recent advances (Heidelberg, 2012), edited by T. Bouganis and O. Venjakob, Contrib. Math. Comput. Sci. 7, Springer, Berlin, 2014. Zbl 06455261

[Waldspurger 1985] J.-L. Waldspurger, "Sur les valeurs de certaines fonctions $L$ automorphes en leur centre de symétrie", Compositio Math. 54:2 (1985), 173-242. MR 87g:11061b Zbl 0567.10021

[Wan 2013] X. Wan, "Iwasawa main conjecture for Hilbert modular forms", preprint, 2013, Available at http://www.math.columbia.edu/ xw2295/Hilbert Modular Forms.pdf.

[Yoshida 1994] H. Yoshida, "On the zeta functions of Shimura varieties and periods of Hilbert modular forms”, Duke Math. J. 75:1 (1994), 121-191. MR 95d:11059 Zbl 0823.11018

[Yoshida 1995] H. Yoshida, "On a conjecture of Shimura concerning periods of Hilbert modular forms”, Amer. J. Math. 117:4 (1995), 1019-1038. MR 96d:11056 Zbl 0841.11024

[Yuan et al. 2013] X. Yuan, S.-W. Zhang, and W. Zhang, The Gross-Zagier formula on Shimura curves, vol. 184, Ann. Math. Stud., Princeton Univ., 2013. MR 3237437 Zbl 1272.11082

[Zarhin 1990] Y. G. Zarhin, " $p$-adic heights on abelian varieties", pp. 317-341 in Séminaire de Théorie des Nombres (Paris, 1987-1988), edited by C. Goldstein, Progr. Math. 81, Birkhäuser, Boston, 1990. MR 91f:11043 Zbl 0707.14040

[Zhang 2001a] S. Zhang, "Heights of Heegner points on Shimura curves", Ann. Math. (2) 153:1 (2001), 27-147. MR 2002g:11081 Zbl 1036.11029

[Zhang 2001b] S.-W. Zhang, "Gross-Zagier formula for GL2", Asian J. Math. 5:2 (2001), 183-290. MR 2003k:11101 Zbl 1111.11030

[Zhang 2004] S.-W. Zhang, "Gross-Zagier formula for GL(2), II", pp. 191-214 in Heegner points and Rankin L-series (Berkeley, CA, 2001), edited by H. Darmon and S.-W. Zhang, Math. Sci. Res. Inst. Publ. 49, Cambridge Univ., 2004. MR 2005k:11121 Zbl 1126.11026

[Zhang 2014] W. Zhang, "Selmer groups and the indivisibility of Heegner points", Camb. J. Math. 2:2 (2014), 191-253. MR 3295917 Zbl 06409349

Communicated by John Henry Coates

Received 2014-09-18 Revised 2015-04-27 Accepted 2015-06-11

daniel.disegni@mcgill.ca

Department of Mathematics and Statistics, McGill University, 805 Shebrooke Street West, Montreal, QC H3A OB9, Canada 


\section{Algebra \& Number Theory}

msp.org/ant

\section{EDITORS}

MANAGING EDITOR

Bjorn Poonen

Massachusetts Institute of Technology

Cambridge, USA

\author{
EDITORIAL BOARD CHAIR \\ David Eisenbud \\ University of California \\ Berkeley, USA
}

BOARD OF EDITORS

Georgia Benkart

Dave Benson

Richard E. Borcherds

John H. Coates

J-L. Colliot-Thélène

Brian D. Conrad

Hélène Esnault

Hubert Flenner

Sergey Fomin

Edward Frenkel

Andrew Granville

Joseph Gubeladze

Roger Heath-Brown

Craig Huneke

Kiran S. Kedlaya

János Kollár

Yuri Manin

Philippe Michel
University of Wisconsin, Madison, USA

University of Aberdeen, Scotland

University of California, Berkeley, USA

University of Cambridge, UK

CNRS, Université Paris-Sud, France

Stanford University, USA

Freie Universität Berlin, Germany

Ruhr-Universität, Germany

University of Michigan, USA

University of California, Berkeley, USA

Université de Montréal, Canada

San Francisco State University, USA

Oxford University, UK

University of Virginia, USA

Univ. of California, San Diego, USA

Princeton University, USA

Northwestern University, USA

École Polytechnique Fédérale de Lausanne
Susan Montgomery

Shigefumi Mori

Raman Parimala

Jonathan Pila

Anand Pillay

Victor Reiner

Peter Sarnak

Joseph H. Silverman

Michael Singer

Vasudevan Srinivas

J. Toby Stafford

Ravi Vakil

Michel van den Bergh

Marie-France Vignéras

Kei-Ichi Watanabe

Efim Zelmanov

Shou-Wu Zhang
University of Southern California, USA

RIMS, Kyoto University, Japan

Emory University, USA

University of Oxford, UK

University of Notre Dame, USA

University of Minnesota, USA

Princeton University, USA

Brown University, USA

North Carolina State University, USA

Tata Inst. of Fund. Research, India

University of Michigan, USA

Stanford University, USA

Hasselt University, Belgium

Université Paris VII, France

Nihon University, Japan

University of California, San Diego, USA

Princeton University, USA

\section{PRODUCTION}

production@msp.org

Silvio Levy, Scientific Editor

See inside back cover or msp.org/ant for submission instructions.

The subscription price for 2015 is US $\$ 255 /$ year for the electronic version, and $\$ 440 /$ year ( $+\$ 55$, if shipping outside the US) for print and electronic. Subscriptions, requests for back issues and changes of subscribers address should be sent to MSP.

Algebra \& Number Theory (ISSN 1944-7833 electronic, 1937-0652 printed) at Mathematical Sciences Publishers, 798 Evans Hall \#3840, c/o University of California, Berkeley, CA 94720-3840 is published continuously online. Periodical rate postage paid at Berkeley, CA 94704, and additional mailing offices.

ANT peer review and production are managed by EditFLOW ${ }^{\circledR}$ from MSP.

\section{PUBLISHED BY}

- mathematical sciences publishers

nonprofit scientific publishing

http://msp.org/

(C) 2015 Mathematical Sciences Publishers 


\section{Algebra \& Number Theory}

Volume $9 \quad$ No. $7 \quad 2015$

Singular moduli that are algebraic units

PHILIPP HABEGGER

Irreducibility of the Gorenstein loci of Hilbert schemes via ray families

GIANFRANCO CASNATI, JOACHIM JELISIEJEW and ROBERTO NOTARI

$p$-adic heights of Heegner points on Shimura curves

DANIEL DISEGNI

Calculabilité de la cohomologie étale modulo $\ell$

David A. Madore and FABrice ORgogozo 